\title{
Supersymmetric Cluster Expansions and Applications to Random Schrödinger Operators
}

\author{
Luca Fresta ${ }^{1,2}$ (iD
}

Received: 29 April 2020 / Accepted: 19 January 2021 / Published online: 24 February 2021

(C) The Author(s) 2021

\begin{abstract}
We study discrete random Schrödinger operators via the supersymmetric formalism. We develop a cluster expansion that converges at both strong and weak disorder. We prove the exponential decay of the disorder-averaged Green's function and the smoothness of the local density of states either at weak disorder and at energies in proximity of the unperturbed spectrum or at strong disorder and at any energy. As an application, we establish Lifshitz-tail-type estimates for the local density of states and thus localization at weak disorder.
\end{abstract}

Keywords Random Schrödinger · Supersymmetric approach · Lifshitz tails

Mathematics Subject Classification (2010) 82B44 · 81T60

\section{Introduction}

In this paper we consider discrete random Schrödinger operators on $\ell^{2}\left(\mathbb{Z}^{\mathrm{D}}\right) \otimes \mathbb{C}^{\mathrm{S}}$ :

$$
H_{\omega}=H+\gamma V_{\omega},
$$

where $H$ is a Hermitian translation-invariant hopping operator, with fast decaying matrix elements, and $V_{\omega}$ is a local random potential, i.e., $\left(V_{\omega} u\right)_{x}=\omega_{x} u_{x},\left\{\omega_{x}\right\}_{x \in \mathbb{Z}^{\mathrm{D}}}$ being i.i.d. random variables with probability distribution $v\left(\mathrm{~d} \omega_{x}\right)$. The set $S \subset \mathbb{N}$ is a finite set of indices, which can possibly represent, e.g., spin or sub-lattice "colour".

Our work focuses on the study of the disorder-averaged Green's function via the supersymmetric (SUSY) formalism. The SUSY approach to random systems was

Luca Fresta

luca.fresta@math.uzh.ch

1 Institute of Mathematics, University of Zurich, Winterthurerstrasse 190, 8057 Zurich, Switzerland

2 Department of Mathematics, University of Tübingen, Auf der Morgenstelle 10, 72076 Tübingen, Germany 
pioneered in the physics literature by Parisi and Sourlas [44, 45], and by Efetov [27] based on the seminal work of Schäfer and Wegner [50, 62]. In the mathematics literature, the SUSY formalism has been rigorously applied in the study of random Schrödinger operators and random matrices, see [14, 15, 18, 19, 21, 23-25, 38, 39, $52-55,58,61]$.

The analysis carried out in this paper is inspired by [6], where SUSY and renormalization group have been used to study a massless hierarchical model for disordered three-dimensional semimetals. One of the main obstacles in the control of the oscillatory SUSY integrals for disordered systems is represented by the presence of mostly complex reference Gaussian "measures". The extension of [6] to the non-hierarchical case requires the use of a cluster expansion that exploits this strong oscillatory nature of the SUSY integrals.

The scope of this work is thus to develop a methodology that will be useful in future settings. The novelty of the present paper consists in the construction of SUSY cluster expansions based on the so-called Battle-Brydges-Federbush formula, see [7, 16], which allows us to expand the disorder-averaged Green's function in terms of tree-graphs. Notice that cluster expansions in the oscillatory SUSY context were previously considered in [15], in the form of a Mayer trick which was applied to the case $H=-\Delta_{\mathbb{Z}^{\mathrm{D}}}$. The resulting expansion is over (connected) graphs, yet convergent because these graphs are made of nearest-neighbour bonds so that their small number can be handled. More generally, one deals with lattice operators with internal degrees of freedom ( $|\mathrm{S}|>1$ ) and with long-range hopping ${ }^{1}$. We believe that adaptations of the methods in [15], though cumbersome, could address the presence of internal degrees of freedom; however, the cluster expansion itself is inherently divergent in the case of long-range hopping operators, because of the large number of (connected) graphs with arbitrary bonds. Our technique can be applied to lattice operators with internal degrees of freedom and with long-range hopping and, as we are going to show, is well suited for handling the weak disorder regime via a dual SUSY representation discussed in Proposition 2.14. As a simple application of our method, we review some known results in the context of random Schrödinger operators.

Let us provide some preliminary definitions. If $\Lambda \subset \mathbb{Z}^{\mathrm{D}}$ is a finite subset, we denote by $H_{\omega, \Lambda}$ the restriction of $H_{\omega}$ to $\ell^{2}(\Lambda) \otimes \mathbb{C}^{S}$ with zero boundary conditions outside of $\Lambda$. The disorder-averaged Green's function at finite volume is the following $\mathbb{C}^{\mathrm{S} \times \mathrm{S}}$-valued function:

$$
\mathbb{G}_{\Lambda}(x, y ; z):=\mathbb{E}_{\omega}\left(\frac{1}{H_{\omega, \Lambda}-z}\right)_{x, y}, \quad z \in \mathbb{C} \backslash \mathbb{R},
$$

where $\mathbb{E}_{\omega}$ denotes the expectation with respect to the product measure $v_{\mathbb{Z}^{\mathrm{D}}}(\mathrm{d} \omega):=$ $\times_{x \in \mathbb{Z}^{\mathrm{D}}} \mathcal{v}\left(\mathrm{d} \omega_{x}\right)$. The local density of states (LDOS) $\rho(E)$ at energy $E \in \mathbb{R}$ can be defined as:

$$
\rho(E):=\lim _{\epsilon \rightarrow 0^{+}} \lim _{\Lambda \mathbb{Z}^{d}} \rho_{\epsilon, \Lambda}(E), \quad \rho_{\epsilon, \Lambda}(E):=\frac{1}{\pi|\mathrm{S}|} \operatorname{Im} \operatorname{Tr}_{\mathbf{S}} \mathbb{G}_{\Lambda}(0,0 ; E+\mathrm{i} \epsilon) .
$$

\footnotetext{
${ }^{1}$ This is the case at weak disorder, since, as we show in Sections 2.4 and 4 , the relevant operator is $(H-$ $E \pm \mathrm{i} \epsilon)^{-1}$, which is not a finite-range lattice operator.
} 
Since $H_{\omega}$ is ergodic, $\rho(E)$ exists for almost $E \in \mathbb{R}$, see [5] and references therein for more details.

We make the following assumptions on the disorder distribution:

(H1) The measure $v$ is Lebesgue absolutely continuous, is even and satisfies the finite-moment condition $\int|\omega|^{|\mathrm{S}|+1} v(\omega) \mathrm{d} \omega<\infty$. This hypothesis is assumed throughout the rest of the script.

(H2) The Fourier transform of the density $v$ satisfies smoothness and decay conditions that will be made precise at separate times, in (H2-I) which is based on Definition 3.1 and in (H2-II) which is based on Definition 4.1. The hypotheses (H2-I) and (H2-II) will be assumed only throughout Section 3 and Section 4 respectively.

We shall remark that we do not anticipate Definitions 3.1 and 4.1 because they require the discussion of Section 2. In fact, assumption (H2) is formulated in terms of superfunctions rather than of $v$ : the connection between the two will only be established in the Appendix.

As will be clear, assumptions (H1) and (H2) are quite restrictive but still include a large class of disorder distributions. This class includes measures with unbounded support like the Gaussian distribution and perturbations of it, but also measures whose density is smooth and compactly supported.

\subsection{Results}

Let us summarize our results based on SUSY cluster expansions and compare them with the literature. Since $v$ is even, w.l.o.g. we shall henceforth restrict to $\gamma>0$. In Section 3 we prove Theorem 3.2 and Corollary 3.4 which respectively establish some properties of $\mathbb{E}_{\omega} G_{\omega, \Lambda}$ and $\rho(E)$ at strong disorder and at any energy. Assuming (H1) and $(\mathrm{H} 2)$ our claims can be informally stated as follows:

\section{Theorem}

(i) Let $H$ be a hopping operator with exponentially decaying matrix elements and let $E \in \mathbb{R}$. If $\gamma \geq C, C$ depending on $E, v$ and the decay of the matrix elements of $H$, then uniformly in $\Lambda$ and in $0 \leq \epsilon \leq 1$

$$
\sup _{\sigma, \sigma^{\prime}}\left|\left(\mathbb{E}_{\omega} G_{\omega, \Lambda}(x, y ; E \pm \mathrm{i} \epsilon)\right)_{\sigma, \sigma^{\prime}}\right| \leq C^{\prime} \gamma^{-2+\delta_{x, y}} \mathrm{e}^{-c|x-y|},
$$

for some constant $C^{\prime}$ depending on $E, v$ and the decay of the matrix elements of $H$, and some constant $c$ depending only on the latter.

(ii) Let $H$ be as above and $\operatorname{Re} E$ be in a bounded set. Under the same assumptions on $\gamma$, the $\operatorname{LDOS} \rho(E)$ is analytic provided that $|\operatorname{Im} E|<c$, c depending on $\gamma$ and $v$.

We remark that Theorem 3.2 also implies the Wegner estimate [63], which in turn implies localization via finite-volume criteria [4].

The exponential decay of the disorder-averaged Green's function for a class of disorder distributions that includes the Gaussian one and uniformly as $\epsilon \rightarrow 0^{+}$is 
novel. In [60] the author says that this is indeed expected to hold based on simple perturbative arguments, but that the corresponding SUSY model is otherwise difficult to analyze because of oscillations. Decay estimates similar to (1.4) are implied, e.g., by the probability estimates in [32] or by fractional moments [3], but the resulting bounds are not uniform down to $\epsilon=0$. In $[57,58]$ a SUSY representation is used to obtain exponential decay, uniformly in $\Lambda$ and $\epsilon$; the technique is based on complex deformation of the "oscillatory measure" and works when the disorder has a Cauchy distribution (or perturbations of it).

Conversely, the regularity properties of the LDOS at strong disorder have been extensively studied, see, e.g., $[15,20,26]$. In [15] the application of cluster expansion techniques to the SUSY representation of the LDOS is pioneered. The authors consider the Laplacian on $\mathbb{Z}^{\mathrm{D}}$ in the presence of a random potential with uniform distribution of the disorder. As anticipated, our analysis relies on a different expansion and applies to a larger class of Hamiltonians: the hopping is long-range and due to the presence of internal degrees of freedom, a non-trivial quartic fermionic interaction appears in the SUSY representation of $\mathbb{E}_{\omega} G_{\omega, \Lambda}$, not present in [15] by the Pauli exclusion principle. Besides, in [15] the constant $C$ is uniform in $E$, which can therefore span the entire real line. The result in (ii) is thus weaker in this regard, but the result in (iv) presented below is complementary and allows us to consider $E$ in unbounded sets at finite $\gamma$.

In Section 4 we prove Theorem 4.2, Corollary 4.4 and Theorem 4.5 which establish some properties of $\mathbb{E}_{\omega} G_{\omega, \Lambda}$ and $\rho(E)$ at weak disorder and at energies outside of the unperturbed spectrum. Assuming (H1) and (H2) our claims can be informally stated as follows:

\section{Theorem}

(iii) Let $E$ be outside of $\sigma(H)$ and define $\delta:=\operatorname{dist}(E, \sigma(H))$. If $\gamma \leq C \delta$ for some constant $C$ depending on $v$, then uniformly in $\Lambda$ and $\epsilon \geq 0$

$$
\sup _{\sigma, \sigma^{\prime}}\left|\left(\mathbb{E}_{\omega} G_{\omega, \Lambda}(x, y ; E \pm \mathrm{i} \epsilon)\right)_{\sigma, \sigma^{\prime}}\right| \leq C^{\prime} \gamma^{-\delta_{x, y}} \mathrm{e}^{-\theta \sqrt{\delta}|x-y|},
$$

for some constant $C^{\prime}$ depending on $v$ and for any $\theta \in[0,1)$.

(iv) Let $\operatorname{Re} E$ be in a bounded set outside of $\sigma(H)$. Under the same assumptions on $\gamma$ as in (iii), the $\operatorname{LDOS} \rho(E)$ is analytic provided that $|\operatorname{Im} E|<c$, $c$ depending on $\gamma$ and $v$.

(v) Under the same assumptions as in (iii), the following bound holds true uniformly in $\Lambda$ and $\epsilon \geq 0$

$$
\left|\rho_{\epsilon, \Lambda}\right| \leq C^{\prime} \gamma^{-1} \mathrm{e}^{-c\left(\gamma \delta^{-1}\right)^{-1 / 2 p}}
$$

for some constants $C^{\prime}$, $c$ and $p$ depending on $v$.

We remark that Lifshitz-tail-type estimates, presented in Theorem 4.5, are wellknown [40] to imply localization via finite-volume criteria, see Remark 4.7 for details.

The exponential decay of the disorder-averaged Green's function at weak disorder and at energies close to the spectrum was expected to hold true [60], but no proof was 
available to the best of our knowledge. One reason for this, is that the methods based on fractional moments or on probability estimates do not allow one to directly control the disorder-averaged Green's function. On the other hand, the SUSY formalism is suitable for studying the disorder-averaged Green's function, but the control of the estimates is cumbersome because of oscillations.

The LDOS was expected to be analytic at weak disorder and away from the unperturbed spectrum [20]. In [14] Bovier studied the analyticity of the LDOS in a hierarchical model at weak Gaussian-distributed disorder and at energies in proximity of the "band edge". The work is based on SUSY and on the renormalization group analysis of the hierarchical Laplacian. Our result applies to any hopping Hamiltonian with quadratic energy dispersion relation at the band edge, e.g., the discrete Laplacian $-\Delta_{\mathbb{Z}^{\mathrm{D}}}$.

Localization in the Lifshitz-tail regime has already been established in [2, 29, 40, 61]. In [2], Aizenman establishes localization up to $\delta \geq C \gamma^{\frac{1}{1+\mathrm{D}+\epsilon}}, \epsilon>0$ and $C$ universal constant. The result was improved by Wang [61] to $\delta \geq \gamma$ and later on boosted by Klopp [40] up to $\delta \geq \gamma^{1+\frac{\mathrm{D}}{4 \mathrm{D}+4}}$. Finally, in [29] Elgart proved localization up to $\delta \geq C_{\text {edge }} \gamma^{2}+\gamma^{4-\epsilon}$, with $\epsilon>0$ and optimal $C_{\text {edge }}>0 .^{2}$ The proof is based on the systematic resummation of the "tadpole diagram" in the perturbative expansion of the Green's function, as analysed by Spencer in [59]. Notice that in [2] a very general class of disorder distribution is considered and localization is established at energies close to the unperturbed spectrum. On the other hand, in [40, 61] and in [29] they consider disorder distributions with semi-bounded and bounded support respectively; furthermore, the result is established at energies close to the spectrum of the random Hamiltonian (in these cases $\delta \equiv \operatorname{dist}\left(E, \sigma\left(H_{\omega}\right)\right)$ ). Our Lifshitz-tail-type estimate in Theorem 4.5 applies to disorder measures with unbounded support, e.g., the Gaussian distribution, and allows us to prove localization in the proximity of the unperturbed spectrum, at energies up to $\delta \geq \gamma|\ln \gamma|^{\alpha}$, for some $\alpha$ sufficiently large. We believe that this is the best achievable result with a single-step SUSY cluster expansion.

The paper is organized as follows. In Section 2 we describe the machinery of the "superformalism" and we provide two SUSY representations of the disorderaveraged Green's function. In Section 3 we formalise the assumptions on $\hat{v}$ as (H2-I), and we prove the results (i) and (ii) above. In Section 4 we introduce the assumption (H2-II) on $\hat{v}$ and we prove the results (iii), (iv) and (v). In appendix we discuss in more detail examples of disorder distributions that satisfy the hypotheses (H2-I) and (H2-II).

\section{SUSY Formalism}

After a brief introduction to normed Grassmann algebras and superfunctions, we state three main propositions that are crucial in our analysis. We conclude the section with Proposition 2.14: we provide two SUSY representations of $\mathbb{G}_{\Lambda}$ that will be used

\footnotetext{
${ }^{2}$ The constant $C_{\text {edge }}$ is precisely the one expected for the mobility edge, and corresponds to the extraction of the tadpole diagram, see [29].
} 
respectively at strong and weak disorder. The use of super Fourier transform and the estimation in norm of the SUSY integrals are the novel features of our method.

\subsection{Normed Grassmann Algebras}

Grassmann algebras formalise the algebraic structure of anticommuting variables. They are widely used in statistical mechanics and field theory [43, 48, 49]. It is useful to equip these algebras with a suitable norm: this will make the estimates in Sections 3 and 4 rather simple and intuitive. Previous examples of the use of norms in the context of Grassmann integration can be found in [8, 30, 31].

Definition 2.1 A Grassmann algebra is a complex unital algebra whose generators anticommute.

We will only consider Grassmann algebras with a finite number of generators. Let $\mathrm{X}, \mathrm{Y}$ be subsets of $\Lambda$; we use the boldface font to denote the Cartesian product of such sets with $\mathrm{S}$, that is, we write $\mathbf{X}:=\mathrm{X} \times \mathrm{S}, \mathbf{Y}:=\mathrm{Y} \times \mathrm{S}$ and so on. We introduce the following Grassmann algebras:

$$
\mathscr{G}:=\bigwedge \mathbb{C}^{\mathrm{S} \times\{ \pm\}}, \quad \mathscr{G}^{\mathrm{X}}:=\bigwedge^{\mathrm{C} \times\{ \pm\}}, \quad \forall \mathrm{X} \subset \Lambda
$$

We notice that $\mathscr{G} \cong \mathscr{G}^{\{x\}}$ for any $x \in \Lambda$, so that any discussion on $\mathscr{G}^{\mathrm{X}}$ includes $\mathscr{G}$ as a special case. Let $\left\{\psi_{x, \sigma}^{\varepsilon}\right\}_{(x, \sigma) \in \mathbf{X}}^{\varepsilon= \pm}$ be the set of generators of $\mathscr{G}^{\mathrm{X}}$ and let the set $\boldsymbol{\Lambda} \times\{ \pm\}$ be provided with a total order. It is easy to see that the Grassmann algebra $\mathscr{G}^{\mathrm{X}}$ is a complex linear space of dimension $2^{2|\mathbf{X}|}$, the basis elements being

$$
\psi^{\mathcal{X}}:=\prod_{(x, \sigma, \varepsilon) \in \mathcal{X}}^{\prime} \psi_{x, \sigma}^{\varepsilon}, \quad \text { for } \quad \mathcal{X} \subset \mathbf{X} \times\{ \pm\},
$$

where the prime in the product operator means that the product is ordered. Accordingly, we can write any element $f \in \mathscr{G}^{\mathrm{X}}$ as

$$
f=\sum_{\mathcal{X} \subset \mathbf{X} \times\{ \pm\}} f_{\mathcal{X}} \psi^{\mathcal{X}},
$$

for some $f_{\mathcal{X}} \in \mathbb{C}$ that will be called coefficients of $f$.

The generators of a Grassmann algebra are often referred to as anticommuting variables. It is useful for our purposes to think of $\mathscr{G}^{\mathrm{X}}$ as a set of functions of such anticommuting variables: if $f \in \mathscr{G}^{\mathrm{X}}$ and if $\left\{\psi_{x, \sigma}^{\varepsilon}\right\}_{(x, \sigma) \in \mathbf{X}}^{\varepsilon= \pm}$ is the set of generators, we will write $f=f(\psi)$. Because we think of elements of a Grassmann algebra as functions of anticommuting variables, it is quite natural to introduce a linear operation like integration. Grassmann integration is the linear operation defined as follows:

$$
\begin{aligned}
& \int \mathrm{d} \psi_{x, \sigma}^{\varepsilon} 1=0, \\
& \int \mathrm{d} \psi_{x, \sigma}^{\varepsilon} \int \mathrm{d} \psi_{x^{\prime}, \sigma^{\prime}}^{\varepsilon^{\prime}} f(\psi)=-\int \mathrm{d} \psi_{x^{\prime}, \sigma^{\prime}}^{\varepsilon^{\prime}} \int \mathrm{d} \psi_{x, \sigma}^{\varepsilon} f(\psi), \\
& \int \mathrm{d} \psi_{x, \sigma}^{\varepsilon}\left(\psi_{x^{\prime}, \sigma^{\prime}}^{\varepsilon^{\prime}} f(\psi)\right)=\delta_{\varepsilon, \varepsilon^{\prime}} \delta_{x, x^{\prime}} \delta_{\sigma, \sigma^{\prime}} f(\psi)-\psi_{x^{\prime}, \sigma^{\prime}}^{\varepsilon^{\prime}} \int \mathrm{d} \psi_{x, \sigma}^{\varepsilon} f(\psi),
\end{aligned}
$$


for any $f(\psi) \in \mathscr{G}^{\mathrm{X}}$. We shall also set

$$
\int \mathrm{d} \psi_{\mathrm{X}} \cdot=\prod_{(x, \sigma) \in \mathbf{X}} \int \mathrm{d} \psi_{x, \sigma}^{+} \int \mathrm{d} \psi_{x, \sigma}^{-} .
$$

Despite looking very peculiar, Grassmann integration is the cornerstone of the supersymmetric formalism, see Proposition 2.6. Furthermore, we will use Grassmann integration as a tool for representing certain maps between elements of a Grassmann algebra, see, e.g., Lemma 2.9. This requires us to work within larger Grassmann algebras generated by two or more sets of independent variables, e.g., $\left\{\psi_{x, \sigma}^{\varepsilon}\right\}_{(x, \sigma) \in \mathbf{X}}^{\varepsilon= \pm}$ and $\left\{\eta_{x, \sigma}^{\varepsilon}\right\}_{(x, \sigma) \in \mathbf{X}}^{\varepsilon= \pm}$. In such cases, we will not explicitly refer to the larger algebra and we will only say that the Grassmann variables are independent.

We now define normed Grassmann algebras, which are introduced already in [9].

Definition 2.2 A Grassmann algebra is said to be normed if it is equipped with a norm $\|\cdot\|$ satisfying:

$$
\|1\|=1, \quad\|f g\| \leq\|f\|\|g\|,
$$

1 denoting the multiplicative identity, $f$ and $g$ being any elements of the Grassmann algebra.

Remark 2.3 Notice that a normed Grassmann algebra is a Banach algebra.

Henceforth, we equip the Grassmann algebra $\mathscr{G}^{\mathrm{X}}$ with the following $\ell^{1}$-type norm:

$$
\|f\|:=\sum_{\mathcal{X} \subset \mathbf{X} \times\{ \pm\}}\left|f_{\mathcal{X}}\right|, \quad \forall f \in \mathscr{G}^{\mathrm{X}} .
$$

Lemma 2.4 The Grassmann algebra $\mathscr{G}^{\mathrm{X}}$ equipped with $\|\cdot\|$ defined in (2.7) is a normed Grassmann algebra.

Remark 2.5 The same holds true for the larger Grassmann algebras with two or more sets of independent generators. In the present work, the norm will be always implicitly associated with the largest Grassmann algebra we work with.

Proof For any $f, g \in \mathscr{G}^{\mathrm{X}}$ we have

$$
\begin{aligned}
& f g=\sum_{\mathcal{X} \subset \mathbf{X} \times\{ \pm\}} \sum_{\mathcal{X}^{\prime} \subset \mathbf{X} \times\{ \pm\}} f_{\mathcal{X}} g_{\mathcal{X}^{\prime}} \psi^{\mathcal{X}} \psi^{\mathcal{X}^{\prime}}
\end{aligned}
$$

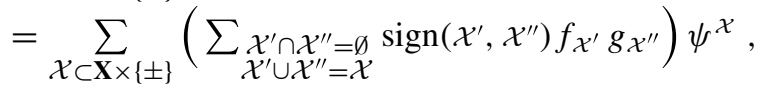

for some $\operatorname{sign}\left(\mathcal{X}^{\prime}, \mathcal{X}^{\prime \prime}\right) \in\{ \pm\}$ which we shall leave unspecified. Hence

$$
\begin{aligned}
\|f g\| & =\sum_{\mathcal{X} \subset \mathbf{X} \times\{ \pm\}}\left|\sum_{\substack{\mathcal{X}^{\prime} \cap \mathcal{X}^{\prime \prime}=\emptyset \\
\mathcal{X}^{\prime} \cup \mathcal{X}^{\prime \prime}=\mathcal{X}}} \operatorname{sign}\left(\mathcal{X}^{\prime}, \mathcal{X}^{\prime \prime}\right) f_{\mathcal{X}^{\prime}} g_{\mathcal{X}^{\prime \prime}}\right| \\
& \leq \sum_{\mathcal{X} \subset \mathbf{X} \times\{ \pm\}} \sum_{\substack{\mathcal{X}^{\prime} \cap \mathcal{X}^{\prime \prime}=\emptyset \\
\mathcal{X}^{\prime} \cup \mathcal{X}^{\prime \prime}=\mathcal{X}}}\left|f_{\mathcal{X}^{\prime}} g_{\mathcal{X}^{\prime \prime}}\right| \leq\|f\|\|g\| .
\end{aligned}
$$


A normed Grassmann algebra is useful because we can estimate Grassmann integrals and thus avoid their exact computation. The following property holds:

$$
\left|\int \mathrm{d} \psi_{\mathrm{X}} f(\psi)\right| \leq\|f\|, \quad \forall f \in \mathscr{G}^{\mathrm{X}},
$$

and will be used extensively below. Notice that when we estimate Grassmann integrals, property (2.6) is particularly useful because the integrand $f(\psi)$ is usually the product of different terms that we want to control separately.

\subsection{Superfields and Superfunctions}

Supervectors are collections of commuting and anticommuting variables: $\Phi:=$ $(\phi, \psi)$,

$$
\phi=\left(\phi_{1,1}, \phi_{1,2} \ldots, \phi_{|\mathrm{S}|, 1}, \phi_{|\mathrm{S}|, 2}\right) \in \mathbb{R}^{2|\mathrm{~S}|}, \quad \psi=\left(\psi_{1}^{+}, \psi_{1}^{-}, \ldots, \psi_{|\mathrm{S}|}^{+}, \psi_{|\mathrm{S}|}^{-}\right),
$$

where $\left\{\psi_{\sigma}^{ \pm}\right\}_{\sigma \in \mathrm{S}}$ is a set of generators of the normed Grassmann algebra $\mathscr{G}$. If $\Phi$ is a supervector, we shall write $\Phi \in \mathcal{S}$. It is customary to introduce the complex-variable notation

$$
\phi_{\sigma}^{ \pm}:=\phi_{\sigma, 1} \pm \mathrm{i} \phi_{\sigma, 2},
$$

that is, $\phi_{\sigma, 1}=\operatorname{Re} \phi_{\sigma}^{+}$and $\phi_{\sigma, 2}=\operatorname{Im} \phi_{\sigma}^{+}$, and to denote by $\Phi^{+}=\left(\phi^{+}, \psi^{+}\right)$and $\Phi^{-}=\left(\phi^{-}, \psi^{-}\right)$the row and column vectors respectively. Thus,

$$
\Phi^{+} \Phi^{-}=\sum_{\sigma \in \mathrm{S}} \phi_{\sigma}^{+} \phi_{\sigma}^{-}+\psi_{\sigma}^{+} \psi_{\sigma}^{-}
$$

is an element of $\mathscr{G}$, while $\Phi^{-} \Phi^{+}$is an $S \times S$ supermatrix.

Functions of a supervector are $\mathscr{G}$-valued maps, $f: \Phi \mapsto f(\Phi) \in \mathscr{G}$. For example, any polynomial in $\Phi^{+} \Phi^{-}$is a function of a supervector; if $f(\cdot)$ is an analytic function, also $f\left(\Phi^{+} \Phi^{-}\right)$, defined by its Taylor expansion, is another instance of such functions.

Superfields are maps from subsets of $\Lambda$ to supervectors, that is $\Phi: \mathrm{X} \ni x \mapsto$ $\Phi_{x} \in \mathcal{S}$. If $\Phi$ is a superfield we shall write $\Phi \in \mathcal{S}^{\mathrm{X}}$. Given a superfield $\Phi$, we define $\Phi^{ \pm}: x \mapsto \Phi_{x}^{ \pm}$. The contraction

$$
\Phi_{x}^{+} A_{x, y} \Phi_{y}^{-}=\sum_{\sigma, \sigma^{\prime} \in \mathrm{S}}\left[\phi_{x, \sigma}^{+}\left(A_{x, y}\right)_{\sigma, \sigma^{\prime}} \phi_{y, \sigma^{\prime}}^{-}+\psi_{x, \sigma}^{+}\left(A_{x, y}\right)_{\sigma, \sigma^{\prime}} \psi_{y, \sigma^{\prime}}^{-}\right],
$$

where $A_{x, y} \in \mathbb{C}^{\mathrm{S} \times \mathrm{S}}$, will be widely used in the rest of the script.

Superfunctions are maps $f: \mathcal{S}^{\mathrm{X}} \rightarrow \mathscr{G}^{\mathrm{X}}$. It is clear that a superfunction can be decomposed as in (2.3):

$$
f(\Phi)=\sum_{\mathcal{X} \subset \mathbf{X} \times\{ \pm\}} f_{\mathcal{X}}(\phi) \psi^{\mathcal{X}}
$$

where the functions $f_{\mathcal{X}}: \mathbb{R}^{2 \mathbf{X}} \rightarrow \mathbb{C}$ will be called the coefficients of $f$. With abuse of notation we will write $f((\phi, 0)):=f_{\emptyset}(\phi)$ or $\left.f(\Phi)\right|_{\psi=0}:=f_{\varnothing}(\phi)$.

We also introduce some useful spaces of superfunctions. We say that a superfunction $f: \mathcal{S}^{\mathrm{X}} \rightarrow \mathscr{G}^{\mathrm{X}}$ belongs to the space $L^{p}\left(\mathcal{S}^{\mathrm{X}}, \mathscr{G}^{\mathrm{X}}\right)$ if all its coefficients belong to 
the Banach space $L^{p}\left(\mathbb{R}^{2 \mathbf{X}}\right)$ of measurable functions $g: \mathbb{R}^{2 \mathrm{X}} \rightarrow \mathbb{C}$ such that $|g|^{p}$ is Lebesgue integrable if $p \in[1, \infty)$ or that are essentially bounded if $p=\infty$. Similarly, we introduce the space of Schwartz superfunctions $\mathscr{S}\left(\mathcal{S}^{\mathrm{X}}, \mathscr{G}^{\mathrm{X}}\right)$, that is, those superfunctions whose coefficients are elements of $\mathscr{S}\left(\mathbb{R}^{2 \mathbf{X}}\right)$. It is natural to consider the following norm on $L^{p}\left(\mathcal{S}^{\mathrm{X}}, \mathscr{G}^{\mathrm{X}}\right)$ for $p \in[1, \infty)$ :

$$
\|f\|_{L^{p}\left(\mathcal{S}^{\mathrm{x}}, \mathscr{G} \mathrm{X}\right)}:=\left(\int \mathrm{d} \phi_{\mathrm{X}}(\|f(\Phi)\|)^{p}\right)^{1 / p}
$$

having set

$$
\mathrm{d} \phi_{x}:=\underset{\sigma \in \mathrm{S}}{\times} \pi^{-1} \mathrm{~d} \phi_{x, \sigma, 1} \mathrm{~d} \phi_{x, \sigma, 2}, \quad \mathrm{~d} \phi_{\mathrm{X}}:=\underset{x \in \mathrm{X}}{\times} \mathrm{d} \phi_{x},
$$

and for $p=\infty$

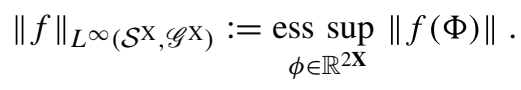

Superintegration is denoted by:

$$
\int \mathrm{d} \Phi_{\mathrm{X}} \cdot:=\int \mathrm{d} \phi_{\mathrm{X}} \int \mathrm{d} \psi_{\mathrm{X}} \cdot
$$

Superintegration will be used as a tool for representing certain maps between superfunctions, e.g., the super Fourier transform, see Definition 2.7. This will require additional superfields and thus other independent Grassmann variables. When this is the case, we always implicitly work in a larger Grassmann algebra, generated by all the Grassmann variables we consider, see also discussion below (2.5), and say that we have independent superfields.

As a simple consequence of (2.10), notice the bounds:

$$
\left|\int \mathrm{d} \Phi_{\mathrm{X}} f(\Phi)\right| \leq \int \mathrm{d} \phi_{\mathrm{X}}\left|\int \mathrm{d} \psi_{\mathrm{X}} f(\Phi)\right| \leq \int \mathrm{d} \phi_{\mathrm{X}}\|f(\Phi)\|=\|f\|_{L^{1}\left(\mathcal{S}^{\mathrm{X}}, \mathscr{G}^{\mathrm{X}}\right)},
$$

which will be repeatedly used in the rest of the script.

\subsection{Three Main Propositions}

The first identity that we present is the so-called supersymmetric replica trick, which is a way to write the entries of a matrix via super Gaussian integrals. This trick was first introduced in the context of random Schrödinger operators by Efetov [27], see also [28] for a review.

Proposition 2.6 Let $A \in \mathbb{C}^{X \times X}$ be a complex matrix with positive definite Hermitian part. The following representation holds true:

$$
\left(A^{-1}\right)_{x, y}=\int \mathrm{d} \Phi_{\mathrm{X}} \mathrm{e}^{-\sum_{x^{\prime}, y^{\prime}} \Phi_{x^{\prime}}^{+} A_{x^{\prime}, y^{\prime}} \Phi_{y^{\prime}}^{-}} \psi_{x}^{-} \psi_{y}^{+}
$$

where $\left(A^{-1}\right)_{x, y} \in \mathbb{C}^{\mathrm{S} \times \mathrm{S}}$ and $\psi_{x}^{-} \psi_{y}^{+}$is a $\mathrm{S} \times \mathrm{S}$ matrix of Grassmann variables. 
Proof It is well known, see for example Section 3 in [64], that for any invertible complex matrix $A \in \mathbb{C}^{\mathbf{X} \times \mathbf{X}}$

$$
\left(A^{-1}\right)_{x, y}=(\operatorname{det} A)^{-1} \int \mathrm{d} \psi_{\mathrm{X}} \mathrm{e}^{-\sum_{x, y \in \mathrm{X}} \psi_{x}^{+} A_{x, y} \psi_{y}^{-}} \psi_{x}^{-} \psi_{y}^{+} .
$$

We notice that if $\phi^{ \pm}=\phi_{1} \pm \mathrm{i} \phi_{2}, \phi_{i} \in \mathbb{R}^{\mathbf{X}}$, we can write

$$
\begin{aligned}
\sum_{x, y \in \mathrm{X}} \phi_{x}^{+} A_{x, y} \phi_{y}^{-} & =\sum_{x, y \in \mathrm{X}} \sum_{\sigma, \sigma^{\prime} \in \mathrm{S}} \phi_{x, \sigma}^{+}\left(A_{x, y}\right)_{\sigma, \sigma^{\prime}} \phi_{y, \sigma^{\prime}}^{-} \\
& =\left(\begin{array}{ll}
\phi_{1}^{T} & \phi_{2}^{T}
\end{array}\right) \underbrace{\left(\begin{array}{cc}
\frac{A+A^{T}}{2} & -\mathrm{i} \frac{A-A^{T}}{2} \\
\mathrm{i} \frac{A-A^{T}}{2} & \frac{A+A^{T}}{2}
\end{array}\right)}_{=: \widetilde{A}}\left(\begin{array}{l}
\phi_{1} \\
\phi_{2}
\end{array}\right),
\end{aligned}
$$

with $\widetilde{A} \in \mathbb{C}^{2 \mathbf{X} \times 2 \mathbf{X}}$ being symmetric. If $A$ has positive Hermitian part then $\tilde{A}$ is nonsingular and $\left(\phi_{1}^{T} \phi_{2}^{T}\right) \operatorname{Re} \tilde{A}\left(\begin{array}{l}\phi_{1} \\ \phi_{2}\end{array}\right) \geq 0$ for any $\phi_{i} \in \mathbb{R}^{\mathbf{X}}$, therefore

$$
\int \mathrm{d} \phi_{\mathrm{X}} \mathrm{e}^{-\sum_{x, y \in \mathrm{X}} \phi_{x}^{+} A_{x, y} \phi_{y}^{-}}=(\operatorname{det} A)^{-1},
$$

see for instance Section 7 in [36]. Putting together (2.22) and (2.24) proves the claim.

In the second proposition we state the super Plancherel identity. This identity is the keystone of the dual SUSY cluster expansion that we present in Section 4. It is based on the theory of super Fourier transform, which we will briefly cover. We shall point out that Berezin had already considered the Fourier transform on Grassmann algebras in his pioneering work [9], see also [10, 11].

Definition 2.7 (Super Fourier Transform) Let $f \in L^{1}\left(\mathcal{S}^{\mathrm{X}}, \mathscr{G}^{\mathrm{X}}\right)$. The super Fourier transform of $f$, denoted by $\widehat{f}$, is the function $\widehat{f}: \mathcal{S}^{\mathrm{X}} \ni \xi \mapsto \widehat{f}(\xi) \in \mathscr{G}^{\mathrm{X}}$ defined by:

$$
\widehat{f}(\xi):=\int \mathrm{d} \Phi_{\mathrm{X}} \mathrm{e}^{-\mathrm{i} \sum_{x \in \mathrm{X}}\left(\xi_{x}^{+} \Phi_{x}^{-}+\Phi_{x}^{+} \xi_{x}^{-}\right)} f(\Phi),
$$

where $\xi=(\kappa, \eta) \in \mathcal{S}^{\mathrm{X}}$ is another independent superfield and where

$$
\xi_{x}^{+} \Phi_{x}^{-}+\Phi_{x}^{+} \xi_{x}^{-}=\sum_{\sigma \in S} \xi_{x, \sigma}^{+} \Phi_{x, \sigma}^{-}+\Phi_{x, \sigma}^{+} \xi_{x, \sigma}^{-}
$$

Some important properties of the Fourier transform on $L^{1}\left(\mathbb{R}^{2 \mathbf{X}}\right)$ and $\mathscr{S}\left(\mathbb{R}^{2 \mathbf{X}}\right)$ carry over to $L^{1}\left(\mathcal{S}^{\mathrm{X}}, \mathscr{G}^{\mathrm{X}}\right)$ and $\mathscr{S}\left(\mathcal{S}^{\mathrm{X}}, \mathscr{G}^{\mathrm{X}}\right)$. In particular, we will see that the super Fourier transform is invertible in the latter space, the inversion being the super Fourier transform with flipped sign.

Proposition 2.8 (Super Plancherel identity) Let $f \in \mathscr{S}\left(\mathcal{S}^{\mathrm{X}}, \mathscr{G}^{\mathrm{X}}\right)$ and $g \in$ $L^{1}\left(\mathcal{S}^{\mathrm{X}}, \mathscr{G}^{\mathrm{X}}\right)$, then

$$
\int \mathrm{d} \Phi_{\mathrm{X}} f(\Phi) g(\Phi)=\int \mathrm{d} \xi_{\mathrm{X}} \widehat{f}(\xi) \widehat{g}(-\xi) .
$$


The proof of this statement is trivial once the inversion theorem for the Grassmann Fourier transform is established.

Lemma 2.9 Let $\left\{\psi_{x, \sigma}^{\varepsilon}\right\}_{(x, \sigma) \in X}^{\varepsilon= \pm}$ and $\left\{\eta_{x, \sigma}^{\varepsilon}\right\}_{(x, \sigma) \in X}^{\varepsilon= \pm}$ be independent Grassmann variables. For any $f=f(\psi)$ let

$$
\widehat{f}(\eta):=\int \mathrm{d} \psi_{\mathrm{X}} \mathrm{e}^{-\mathrm{i} \sum_{x \in \mathrm{X}}\left(\eta_{x}^{+} \psi_{x}^{-}+\psi_{x}^{+} \eta_{x}^{-}\right)} f(\psi),
$$

be the Grassmann Fourier transform of $f$. Then,

$$
f(\psi)=\int \mathrm{d} \psi_{\mathrm{X}} \mathrm{e}^{\mathrm{i} \sum_{x \in \mathrm{X}}\left(\psi_{x}^{+} \eta_{x}^{-}+\eta_{x}^{+} \psi_{x}^{-}\right)} \widehat{f}(\eta) .
$$

Proof Let $\left\{\psi_{x, \sigma}^{\varepsilon}\right\}_{(x, \sigma) \in \mathbf{X}}^{\varepsilon= \pm},\left\{\psi_{x, \sigma}^{\varepsilon}\right\}_{(x, \sigma) \in \mathbf{X}}^{\varepsilon= \pm}$ and $\left\{\eta_{x, \sigma}^{\varepsilon}\right\}_{(x, \sigma) \in \mathbf{X}}^{\varepsilon= \pm}$ be independent Grassmann variables. Define

$$
\delta^{\mathrm{X}}(\psi):=\int \mathrm{d} \eta_{\mathrm{X}} \mathrm{e}^{-\mathrm{i} \sum_{x \in \mathrm{X}}\left(\eta_{x}^{+} \psi_{x}^{-}+\psi_{x}^{+} \eta_{x}^{-}\right)} .
$$

We are going to show that this function plays the role of the Dirac delta function in the anticommuting setting, that is

$$
\int \mathrm{d} \psi_{\mathrm{X}} \delta^{\mathrm{X}}\left(\psi-\psi^{\prime}\right) f(\psi)=f\left(\psi^{\prime}\right)
$$

By linearity, it suffices to prove (2.31) in the case $f(\psi)=\psi^{\mathcal{X}}$, for any $\mathcal{X} \subset(\mathbf{X} \times$ $\{ \pm\})$. First of all, we notice that by nilpotency

$$
\mathrm{e}^{-\mathrm{i} \sum_{x \in \mathrm{X}}\left(\eta_{x}^{+} \psi_{x}^{-}+\psi_{x}^{+} \eta_{x}^{-}\right)}=\prod_{(x, \sigma, \varepsilon) \in \mathbf{X} \times\{ \pm\}}\left(1-\mathrm{i} \varepsilon \eta_{x, \sigma}^{\varepsilon} \psi_{x, \sigma}^{-\varepsilon}\right) .
$$

The term which contains all the $\eta$ 's is by inspection $\prod_{(x, \sigma)} \eta_{x, \sigma}^{-} \eta_{x, \sigma}^{+} \psi_{x, \sigma}^{-} \psi_{x, \sigma}^{+}$, thus

$$
\delta^{\mathrm{X}}(\psi)=\int \mathrm{d} \eta_{\mathrm{X}} \mathrm{e}^{-\mathrm{i} \sum_{x \in \mathrm{X}}\left(\eta_{x}^{+} \psi_{x}^{-}+\psi_{x}^{+} \eta_{x}^{-}\right)}=\prod_{(x, \sigma) \in \mathbf{X}} \psi_{x, \sigma}^{-} \psi_{x, \sigma}^{+},
$$

where we used that $\eta_{x, \sigma}^{-} \eta_{x, \sigma}^{+}$and $\psi_{x, \sigma}^{-} \psi_{x, \sigma}^{+}$are even elements and thus commute. As a consequence, for any set $\mathcal{X} \subset(\mathbf{X} \times\{ \pm\})$ we can write

$$
\delta^{X}(\psi)=\operatorname{sign}(\mathcal{X}) \psi^{\mathcal{X}^{\prime}} \psi^{\mathcal{X}}
$$

where $\mathcal{X}^{\prime}:=(\mathbf{X} \times\{ \pm\}) \backslash \mathcal{X}$ and where $\operatorname{sign}(\mathcal{X})$ is a permutation sign which we leave unspecified. Again by nilpotency, we notice that the only term in $\delta^{X}\left(\psi-\psi^{\prime}\right)$ that gives non-vanishing contribution to (2.31) with $f(\psi)=\psi^{\mathcal{X}}$ is $\operatorname{sign}(\mathcal{X}) \psi^{\mathcal{X}^{\prime}}\left(-\psi^{\prime}\right)^{\mathcal{X}}$, so that

$$
\begin{aligned}
\int \mathrm{d} \psi_{\mathrm{X}} \delta^{\mathrm{X}}\left(\psi-\psi^{\prime}\right) \psi^{\mathcal{X}} & =\int \mathrm{d} \psi_{\mathrm{X}} \operatorname{sign}(\mathcal{X}) \psi^{\mathcal{X}^{\prime}}\left(-\psi^{\prime}\right)^{\mathcal{X}} \psi^{\mathcal{X}} \\
& =\int \mathrm{d} \psi_{\mathrm{X}}\left(\prod_{(x, \sigma) \in \mathbf{X}} \psi_{x, \sigma}^{-} \psi_{x, \sigma}^{+}\right) \psi^{\prime \mathcal{X}}=\psi^{\prime \mathcal{X}}
\end{aligned}
$$


where in the second equality we used that $\left(-\psi^{\prime}\right)^{\mathcal{X}} \psi^{\mathcal{X}}=\psi^{\mathcal{X}}\left(\psi^{\prime}\right)^{\mathcal{X}}$ while in the last one the definition of $\int \mathrm{d} \psi_{\mathrm{X}}$. The inversion of the Grassmann Fourier transform is then established:

$$
\begin{aligned}
& \int \mathrm{d} \eta_{\mathrm{X}} \mathrm{e}^{\mathrm{i} \sum_{x \in \mathrm{X}}\left(\psi_{x}^{+} \eta_{x}^{-}+\eta_{x}^{+} \psi_{x}^{-}\right) \widehat{f}(\eta)} \\
= & \int \mathrm{d} \psi_{\mathrm{X}}^{\prime} \int \mathrm{d} \eta_{\mathrm{X}} \mathrm{e}^{-\mathrm{i} \sum_{x \in \mathrm{X}}\left(\eta_{x}^{+}\left(\psi_{x}^{\prime-}-\psi_{x}^{-}\right)+\left(\psi_{x}^{\prime+}-\psi_{x}^{+}\right) \eta_{x}^{-}\right)} f\left(\psi^{\prime}\right) \\
= & \int \mathrm{d} \psi_{\mathrm{X}}^{\prime} \delta^{\mathrm{X}}\left(\psi^{\prime}-\psi\right) f\left(\psi^{\prime}\right)=f(\psi) .
\end{aligned}
$$

Proof of Proposition 2.8 By swapping the bosonic integration with Grassmann integration and using the invertibility of the Fourier transform in $\mathscr{S}\left(\mathbb{R}^{2 \mathbf{X}}, \mathbb{C}\right)$ we obtain that

$$
f(\Phi)=\int \mathrm{d} \xi_{\mathrm{X}} \mathrm{e}^{\mathrm{i} \sum_{x \in \mathrm{X}}\left(\xi_{x}^{+} \Phi_{x}^{-}+\Phi_{x}^{+} \xi_{x}^{-}\right)} \widehat{f}(\xi),
$$

for any $f \in \mathscr{S}\left(\mathcal{S}^{\mathrm{X}}, \mathscr{G}^{\mathrm{X}}\right)$, thus establishing the invertibility of the super Fourier transform on this space. We have,

$$
\begin{aligned}
\int \mathrm{d} \Phi_{\mathrm{X}} f(\Phi) g(\Phi) & =\int \mathrm{d} \Phi_{\mathrm{X}} \int \mathrm{d} \xi_{\mathrm{X}} \mathrm{e}^{\mathrm{i} \sum_{x \in \mathrm{X}}\left(\xi_{x}^{+} \Phi_{x}^{-}+\Phi_{x}^{+} \xi_{x}^{-}\right)} \widehat{f}(\xi) g(\Phi) \\
& =\int \mathrm{d} \xi_{\mathrm{X}} \widehat{f}(\xi) \widehat{g}(-\xi),
\end{aligned}
$$

where we swapped the superintegrals by the Fubini-Tonelli theorem, since $\|\widehat{f}\|_{L^{1}(\mathcal{S} \mathrm{x}, \mathscr{G} \mathrm{x})},\|g\|_{L^{1}(\mathcal{S} \mathrm{x}, \mathscr{G} \mathrm{x})}$ and $\left\|\mathrm{e}^{\mathrm{i} \sum_{x \in \mathrm{X}}\left(\xi_{x}^{+} \Phi_{x}^{-}+\Phi_{x}^{+} \xi_{x}^{-}\right)}\right\|_{L^{\infty}\left(\mathcal{S}^{\mathrm{x}}, \mathscr{G} \mathrm{x}\right)}$ are finite.

Before moving on to the last proposition of this section, we present a lemma that is the extension of a simple and well-known inequality in the theory of Fourier transform.

Lemma 2.10 Let $f \in L^{1}\left(\mathcal{S}^{\mathrm{X}}, \mathscr{G}^{\mathrm{X}}\right)$. The following bound holds true:

$$
\|\widehat{f}\|_{L^{\infty}\left(\mathcal{S}^{\mathrm{x}}, \mathscr{G} \mathrm{X}\right)} \leq\|f\|_{L^{1}\left(\mathcal{S}^{\mathrm{x}}, \mathscr{G} \mathrm{X}\right)} .
$$

Proof Let $\Phi=(\phi, \psi) \in \mathcal{S}^{\mathrm{X}}$ and $\xi=(\kappa, \eta) \in \mathcal{S}^{\mathrm{X}}$ be independent superfields. Clearly we have

$$
\|\widehat{f}(\xi)\| \leq \int \mathrm{d} \phi_{\mathrm{X}}\left\|\int \mathrm{d} \psi_{\mathrm{X}} \mathrm{e}^{-\mathrm{i} \sum_{x \in \mathrm{X}}\left(\eta^{+} \psi_{x}^{-}+\psi_{x}^{+} \eta_{x}^{-}\right)} f(\Phi)\right\| .
$$

We shall prove that the Grassmann norm on the r.h.s. of (2.40) is equal to $\|f(\Phi)\|$ by showing that the Grassmann Fourier transform rearranges the coefficients up to a phase factor. We write the exponential term as

$$
\prod_{(x, \sigma, \varepsilon) \in \mathbf{X} \times\{ \pm\}}\left(1-\mathrm{i} \varepsilon \eta_{x, \sigma}^{\varepsilon} \psi_{x, \sigma}^{-\varepsilon}\right)=\sum_{\mathcal{X} \subset \mathbf{X} \times\{ \pm\}} \operatorname{phase}(\mathcal{X}) \eta^{\mathcal{X}} \psi^{\overline{\mathcal{X}}}
$$


where phase $(\mathcal{X}) \in U(1)$, while $\overline{\mathcal{X}}$ is the subset of $\mathbf{X} \times\{ \pm\}$ obtained from $\mathcal{X}$ by flipping all the $\varepsilon$ 's. By noticing that $\delta^{\mathrm{X}}(\psi)=\operatorname{sign}\left(\overline{\mathcal{X}}^{\prime}\right) \psi^{\overline{\mathcal{X}}} \psi^{\overline{\mathcal{X}}^{\prime}}$, where $\overline{\mathcal{X}}^{\prime}=(\mathbf{X} \times\{ \pm\}) \backslash \overline{\mathcal{X}}$ (see proof of Lemma 2.9) we obtain

$$
\int \mathrm{d} \psi_{\mathrm{X}} \mathrm{e}^{-\mathrm{i} \sum_{x \in \mathrm{X}}\left(\eta^{+} \psi_{x}^{-}+\psi_{x}^{+} \eta_{x}^{-}\right)} f(\Phi)=\sum_{\mathcal{X} \subset \mathbf{X} \times\{ \pm\}} \operatorname{phase}(\mathcal{X}) \operatorname{sign}\left(\overline{\mathcal{X}}^{\prime}\right) f_{\overline{\mathcal{X}}^{\prime}}(\phi) \eta^{\overline{\mathcal{X}}^{\prime}},
$$

and the claim follows since there is a one to one correspondence between $\mathcal{X}$ and $\overline{\mathcal{X}}^{\prime}$.

Last but not least, supersymmetry is a crucial property in the analysis of superintegrals. The last proposition we present is an instance of the well-known localization formula for supersymmetric functions [44].

Let us first define what supersymmetry is.

Definition 2.11 (SUSY) Introduce the differential operator:

$$
Q_{\Phi}=\sum_{(x, \sigma) \in \mathbf{X}} \sum_{\varepsilon}\left[\psi_{x, \sigma}^{\varepsilon} \frac{\partial}{\partial \phi_{x, \sigma}^{\varepsilon}}-\varepsilon \phi_{x, \sigma}^{\varepsilon} \frac{\partial}{\partial \psi_{x, \sigma}^{\varepsilon}}\right] .
$$

We say that $f$ is supersymmetric if it is $Q$-closed, that is, it is differentiable and satisfies

$$
Q_{\Phi} f(\Phi)=0
$$

For the sake of generality, we shall state the SUSY localization formula under weak decay assumptions.

Proposition 2.12 (SUSY localization formula) Let $f$ be supersymmetric and decaying at infinity. If $f$ and $\Phi \mapsto\left(1+\left|\phi_{x, \sigma}^{\varepsilon}\right|\right)^{-1} \psi_{x, \sigma}^{\varepsilon}\left(\partial / \partial \phi_{x, \sigma}^{\varepsilon}\right) f(\Phi)$ are in $L^{1}\left(\mathcal{S}^{\mathrm{X}}, \mathscr{G}^{\mathrm{X}}\right)$ for any $(x, \sigma, \varepsilon) \in \boldsymbol{X} \times\{ \pm\}$ then

$$
\int \mathrm{d} \Phi_{\mathrm{X}} f(\Phi)=f(0)
$$

Even though our assumptions are somewhat weaker than, e.g., [8, 25], the proof outlined in those references carries over and is therefore here omitted. A more geometrical perspective on this statement can be found in [12, 13, 51].

The following lemma will be useful for the application of the SUSY localization formula.

Lemma 2.13 Let $f \in L^{1}(\mathcal{S}, \mathscr{G})$ be even, supersymmetric and invariant under $U(1)^{\times \mathrm{S}}$ fermionic transformations, $\psi_{\sigma}^{\varepsilon} \mapsto \mathrm{e}^{\mathrm{i} \varepsilon \theta_{\sigma}} \psi_{\sigma}^{\varepsilon}$ for $\theta \in[0,2 \pi)^{\mathrm{S}}$. Then

$$
\psi_{\sigma}^{\varepsilon} \frac{\partial}{\partial \phi_{\sigma}^{\varepsilon}} f(\Phi)=-\varepsilon \phi_{\sigma}^{-\varepsilon} \frac{\partial}{\partial \psi_{\sigma}^{-\varepsilon}} f(\Phi), \quad \forall \sigma \in \mathrm{S}, \varepsilon \in\{ \pm\}
$$

Proof Denote by $\mathcal{U}_{\theta}$ the $U(1)^{\times S}$ fermionic transformation. Since 0 and $f$ are invariant, we have that $\mathcal{U}_{\theta} Q_{\Phi} \mathcal{U}_{\theta}^{-1} f(\Phi)=0$ for any $\theta \in[0,2 \pi)^{\mathrm{S}}$. We decompose $Q_{\Phi}=$ 
$\sum_{\varepsilon, \sigma} \widetilde{Q}_{\varepsilon, \sigma}$, with $\widetilde{Q}_{\varepsilon, \sigma}:=\psi_{\sigma}^{\varepsilon} \frac{\partial}{\partial \phi_{\sigma}^{\varepsilon}}+\varepsilon \phi_{\sigma}^{-\varepsilon} \frac{\partial}{\partial \psi_{\sigma}^{-\varepsilon}}$ satisfying $\mathcal{U}_{\theta} \widetilde{Q}_{\varepsilon, \sigma} \mathcal{U}_{\theta}^{-1}=\mathrm{e}^{\mathrm{i} \varepsilon \theta_{\sigma}} \widetilde{Q}_{\varepsilon, \sigma}$.

Fix $\bar{\sigma}$ and $\bar{\varepsilon}$ and choose $\theta_{\sigma}=\delta_{\bar{\sigma}, \sigma} \pi$ and $\theta_{\sigma}^{\prime}=\delta_{\bar{\sigma}, \sigma} \pi / 2$. Then, the linear combination

$$
\begin{aligned}
0= & \frac{1}{4} Q_{\Phi} f(\Phi)-\frac{1}{4} \mathcal{U}_{\theta} Q_{\Phi} \mathcal{U}_{\theta}^{-1} f(\Phi) \\
& -\frac{\mathrm{i}}{4} \bar{\varepsilon} \mathcal{U}_{\theta^{\prime}} Q_{\Phi} \mathcal{U}_{\theta^{\prime}}^{-1} f(\Phi)+\frac{\mathrm{i}}{4} \bar{\varepsilon} \mathcal{U}_{\theta+\theta^{\prime}} Q_{\Phi} \mathcal{U}_{\theta+\theta^{\prime}}^{-1} f(\Phi)=\widetilde{Q}_{\bar{\varepsilon}, \bar{\sigma}} f(\Phi),
\end{aligned}
$$

is exactly (2.46) for $\bar{\sigma}$ and $\bar{\varepsilon}$.

\subsection{Disorder-Averaged Green'S Function}

In the proposition below two SUSY representations of the disorder-averaged Green's function are finally discussed. The first representation is well-known and has already been applied to the study of the Anderson model at strong disorder [27], see also [15]. The second representation is new, to the best of our knowledge. It is particularly useful at weak disorder and energies close to the spectrum of $H$. We call these two SUSY representations respectively "direct SUSY integral" and "dual SUSY integral".

We provide some preliminary definitions. By assumption on the disorder distribution, see (H1), we have that $\int|\omega|^{|S|+1} v(\omega) \mathrm{d} \omega<\infty$ and thus we can define the following function of a supervector:

$$
F_{z}(\Phi):=\mathrm{e}^{\gamma^{-1} z\left(\Phi^{+} \Phi^{-}\right)} \hat{v}\left(\Phi^{+} \Phi^{-}\right),
$$

where $z \in \mathbb{C}$ and where

$$
\hat{v}\left(\Phi^{+} \Phi^{-}\right):=\int \mathrm{e}^{\mathrm{i} \omega \Phi^{+} \Phi^{-}} \nu(\omega) \mathrm{d} \omega=\sum_{n=0}^{|\mathrm{S}|} \hat{v}^{(n)}\left(\phi^{+} \phi^{-}\right) \frac{\left(\psi^{+} \psi^{-}\right)^{n}}{n !} .
$$

The condition on the moments of $v$ is necessary in order to have $\hat{v}^{(n)}$ well-defined for $n=0, \ldots,|\mathrm{S}|$. It is important to notice that $F_{z}$ is supersymmetric $Q_{\Phi} F_{z}(\Phi)=0$, even $F_{z}(\Phi)=F_{z}(-\Phi)$ and invariant under $U(1)^{\times S}$ fermionic transformations, see Lemma 2.13.

We will also need to measure the decay of the lattice operators we consider. To this end, we introduce the following norm: for any $A \in \mathbb{C}^{\left(\mathbb{Z}^{\mathrm{D}} \times \mathrm{S}\right) \times\left(\mathbb{Z}^{\mathrm{D}} \times \mathrm{S}\right)}$

$$
\|A\|_{\infty, 1}:=\sup _{x \in \mathbb{Z}^{\mathrm{D}}} \sum_{y \in \mathbb{Z}^{\mathrm{D}}} \sum_{\sigma, \sigma^{\prime} \in \mathrm{S}}\left|\left(A_{x, y}\right)_{\sigma, \sigma^{\prime}}\right| .
$$

We can finally state the proposition.

Proposition 2.14 (SUSY representation) Let $\Lambda \subset \mathbb{Z}^{\mathrm{D}}$ be finite and $\epsilon>0$. Assume (HI) and that $F_{0} \in L^{1}(\mathcal{S}, \mathscr{G})$. Then for any $\gamma>0$, and any $x, y \in \Lambda$ the functions $\mathbb{G}_{\Lambda}(x, y ; E+\mathrm{i} \epsilon)$ and $\mathbb{G}_{\Lambda}(x, y ; E-\mathrm{i} \epsilon)$ are analytic respectively in $\operatorname{Im} E>0$ and in $\operatorname{Im} E<0$, and the following representations hold true:

(i) Direct SUSY integral:

$$
\mathbb{G}_{\Lambda}(x, y ; E \pm \mathrm{i} \epsilon)= \pm \mathrm{i} \gamma^{-1} \int \mathrm{d} \mu_{\Lambda}^{ \pm}(\Phi) F_{ \pm \mathrm{i} E-\epsilon}^{\Lambda}(\Phi) \psi_{x}^{-} \psi_{y}^{+},
$$


where for any $\mathrm{X} \subset \Lambda$ we define:

$$
\begin{aligned}
\mathrm{d} \mu_{\mathrm{X}}^{ \pm}(\Phi) & =\mathrm{d} \Phi_{\mathrm{X}} \mu_{\mathrm{X}}^{ \pm}(\Phi), \mu_{\mathrm{X}}^{ \pm}(\Phi):=\mathrm{e}^{\mp \mathrm{i} \gamma^{-1} \sum_{x, y \in \mathrm{X}} \Phi_{x}^{+} H_{x, y} \Phi_{y}^{-},} \\
F_{ \pm \mathrm{i} E-\epsilon}^{\mathrm{X}}(\Phi) & =\prod_{x \in \mathrm{X}} F_{ \pm \mathrm{i} E-\epsilon}\left(\Phi_{x}\right) .
\end{aligned}
$$

(ii) Dual SUSY integral:

$$
\mathbb{G}_{\Lambda}(x, y ; E \pm \mathrm{i} \epsilon)= \pm \mathrm{i} \gamma^{-1} \int \mathrm{d} \widehat{\mu}_{\Lambda}^{ \pm}(\xi) \frac{\partial}{\partial \eta_{x}^{+}} \frac{\partial}{\partial \eta_{y}^{-}} \widehat{F}_{0}^{\Lambda}(\xi),
$$

where for any $\mathrm{X} \subset \Lambda$ we define:

$$
\begin{aligned}
\mathrm{d} \widehat{\mu}_{\mathrm{X}}^{ \pm}(\xi) & =\mathrm{d} \xi \mathrm{X} \widehat{\mu}_{\mathrm{X}}^{ \pm}(\xi), \widehat{\mu}_{\mathrm{X}}^{ \pm}(\xi):=\mathrm{e}^{ \pm \mathrm{i} \gamma \sum_{x, y \in \mathrm{X}} \xi_{x}^{+} C_{x, y}^{E \pm \mathrm{i} \epsilon} \xi_{y}^{-}}, \\
\widehat{F}_{0}^{\mathrm{X}}(\xi) & =\prod_{x \in \mathrm{X}} \widehat{F}_{0}\left(\xi_{x}\right),
\end{aligned}
$$

with $C^{z}=(H-z)^{-1}$ and $\xi=(\kappa, \eta) \in \mathcal{S}^{\Lambda}$, see below (2.25).

Proof We apply Proposition 2.6 to $\pm \mathrm{i}\left(H_{\omega}-E \mp \mathrm{i} \epsilon\right) \in \mathbb{C}^{\Lambda \times \Lambda}$, which has positive definite Hermitian part for any $\omega\left(H_{\omega}\right.$ is Hermitian) provided that $\epsilon>0$. After rescaling $\Phi \rightarrow \gamma^{1 / 2} \Phi$ (which preserves $\mathrm{d} \Phi_{\Lambda}$, see, e.g., [9]) we can write:

$$
\pm \mathrm{i}\left(\left(H_{\omega, \Lambda}-E \mp \mathrm{i} \epsilon\right)^{-1}\right)_{x, y}=\gamma^{-1} \int \mathrm{d} \mu_{\Lambda}^{ \pm}(\Phi) \psi_{x}^{-} \psi_{y}^{+} \mathrm{e}^{\mp \mathrm{i} \sum_{x^{\prime}}\left[\omega_{x^{\prime}}-\gamma^{-1}(E \pm \mathrm{i} \epsilon)\right] \Phi_{x^{\prime}}^{+} \Phi_{x^{\prime}}^{-}}
$$

where $\psi_{x}^{-} \psi_{y}^{+}$is an $\mathrm{S} \times \mathrm{S}$ matrix of Grassmann variables and where $\mathrm{d} \mu_{\Lambda}^{ \pm}(\Phi)$ is as in the statement. We shall swap disorder-expectation with superintegration by the Fubini-Tonelli theorem. To this end, we need to prove that

$$
\sup _{\sigma, \sigma^{\prime}} \mathbb{E}_{\omega} \int \mathrm{d} \phi_{\Lambda}\left|\int \mathrm{d} \psi_{\Lambda} \mu_{\Lambda}^{ \pm}(\Phi) \psi_{x, \sigma}^{-} \psi_{y, \sigma^{\prime}}^{+} \mathrm{e}^{\mp \mathrm{i} \sum_{x^{\prime}}\left[\omega_{x^{\prime}}-\gamma^{-1}(E \pm \mathrm{i} \epsilon)\right] \Phi_{x^{\prime}}^{+} \Phi_{x^{\prime}}^{-}}\right|<\infty \text {. }
$$

By means of (2.10), see also (2.20), we have that

1.h.s. (2.56)

$$
\leq \sup _{\sigma, \sigma^{\prime}} \mathbb{E}_{\omega} \int \mathrm{d} \phi_{\Lambda} \| \mu_{\Lambda}^{ \pm}(\Phi) \psi_{x, \sigma}^{-} \psi_{y, \sigma^{\prime}}^{+} \mathrm{e}^{\mp \mathrm{i} \sum_{x^{\prime}}\left[\omega_{x^{\prime}}-\gamma^{-1}(E \pm \mathrm{i} \epsilon)\right] \Phi_{x^{\prime}}^{+} \Phi_{x^{\prime}}^{-} \| .}
$$

Since $\Lambda$ is finite, for any hopping Hamiltonian the following quantity is bounded

$$
\left\|H_{\Lambda}\right\|_{\infty, 1}=\sup _{x \in \Lambda} \sum_{x^{\prime} \in \Lambda} \sum_{\sigma, \sigma^{\prime} \in \mathrm{S}}\left|\left(H_{x, x^{\prime}}\right)_{\sigma, \sigma^{\prime}}\right|<\infty,
$$

see (2.50), where $H_{\Lambda}=\mathbf{1}_{\Lambda} H \mathbf{1}_{\Lambda}, \mathbf{1}_{\Lambda}$ being the characteristic function of $\Lambda$. It follows that we can bound the Grassmann norm of $\mu_{\Lambda}^{ \pm}(\Phi)$ as follows:

$$
\begin{aligned}
\left\|\mu_{\Lambda}^{ \pm}(\Phi)\right\| & =\left\|\mathrm{e}^{\mp \mathrm{i} \gamma^{-1} \sum_{x, y \in \Lambda} \phi_{x}^{+} H_{x, y} \phi_{y}^{-}} \mathrm{e}^{\mp \mathrm{i} \gamma^{-1} \sum_{x, y \in \Lambda} \psi_{x}^{+} H_{x, y} \psi_{y}^{-}}\right\| \\
& \leq \mathrm{e}^{\gamma^{-1}\left\|\sum_{x, y \in \Lambda} \psi_{x}^{+} H_{x, y} \psi_{y}^{-}\right\|} \leq \mathrm{e}^{\gamma^{-1}\left\|H_{\Lambda}\right\|_{\infty, 1}|\Lambda|},
\end{aligned}
$$

where we used the properties of the norm and that $\sum_{x, y \in \Lambda} \phi_{x}^{+} H_{x, y} \phi_{y}^{-} \in \mathbb{R}$. 
If we denote by Tay ${ }_{n}$ the $n$-th order Taylor expansion in zero, then the following bound

$$
\begin{aligned}
& \sup _{\sigma, \sigma^{\prime}} \| \mu_{\Lambda}^{ \pm}(\Phi) \psi_{x, \sigma}^{-} \psi_{y, \sigma^{\prime}}^{+} \mathrm{e}^{\mp \mathrm{i} \sum_{x^{\prime}}\left[\omega_{x^{\prime}}-\gamma^{-1}(E \pm \mathrm{i} \epsilon)\right] \Phi_{x^{\prime}}^{+} \Phi_{x^{\prime}}^{-} \|} \\
& \leq\left\|\mu_{\Lambda}^{ \pm}(\Phi)\right\| \sup _{\sigma, \sigma^{\prime}}\left\|\psi_{x, \sigma}^{-} \psi_{y, \sigma^{\prime}}^{+}\right\| \prod_{x^{\prime} \in \Lambda}\left\|\mathrm{e}^{\mp \mathrm{i}\left[\omega_{x^{\prime}}-\gamma^{-1}(E \pm \mathrm{i} \epsilon)\right] \Phi_{x^{\prime}}^{+} \Phi_{x^{\prime}}^{-}}\right\| \\
& \leq \mathrm{e}^{\gamma^{-1}\left\|H_{\Lambda}\right\|_{\infty, 1}|\Lambda|}\left[\prod_{x^{\prime} \in \Lambda} \mathrm{e}^{-\epsilon \gamma^{-1} \phi_{x^{\prime}}^{+} \phi_{x^{\prime}}^{-} \|} \mathrm{e}^{\mp \mathrm{i}\left[\omega_{x^{\prime}}-\gamma^{-1}(E \pm \mathrm{i} \epsilon)\right] \psi_{x^{\prime}}^{+} \psi_{x^{\prime}}^{-}} \|\right] \\
& \leq \mathrm{e}^{\gamma^{-1}\left\|H_{\Lambda}\right\|_{\infty, 1}|\Lambda|}\left[\prod_{x^{\prime} \in \Lambda} \mathrm{e}^{-\epsilon \gamma^{-1} \phi_{x^{\prime}}^{+} \phi_{x^{\prime}}^{-}} \operatorname{Tay}_{|\mathrm{S}|} \mathrm{e}^{\left|\omega_{x^{\prime}}-\gamma^{-1}(E \pm \mathrm{i} \epsilon) \| \mathrm{S}\right|}\right]
\end{aligned}
$$

proves the finiteness of the r.h.s. in (2.57), since

$$
\begin{aligned}
& \mathbb{E}_{\omega} \prod_{x^{\prime} \in \Lambda} \operatorname{Tay}_{|\mathrm{S}|} \mathrm{e}^{\left|\omega_{x^{\prime}}-\gamma^{-1}(E \pm \mathrm{i} \epsilon)\right||\mathrm{S}|}<\infty, \\
& \int \mathrm{d} \phi_{\Lambda} \prod_{x^{\prime} \in \Lambda} \mathrm{e}^{-\epsilon \gamma^{-1} \phi_{x^{\prime}}^{+} \phi_{x^{\prime}}^{-}}=\left(\gamma \epsilon^{-1}\right)^{|\Lambda||\mathrm{S}|},
\end{aligned}
$$

respectively by assumption (H1) and by explicit computation. Then the claim in (i) follows because

$$
\mathbb{E}_{\omega} \prod_{x^{\prime} \in \Lambda} \mathrm{e}^{\mp \mathrm{i} \omega_{x^{\prime}} \Phi_{x^{\prime}}^{+} \Phi_{x^{\prime}}^{-}}=\prod_{x^{\prime}} \hat{v}\left(\Phi_{x^{\prime}}^{+} \Phi_{x^{\prime}}^{-}\right)
$$

where we used that $\left\{\omega_{x}\right\}_{x \in \Lambda}$ are i.i.d. and $v$ is even.

Next, we prove the analyticity of $\mathbb{G}_{\Lambda}(x, y ; E \pm \mathrm{i} \epsilon)$ by showing that the r.h.s. of (2.51) is analytic in the relevant domain. This is achieved by application of the Fubini-Tonelli and Morera's theorem. First of all, we notice that the Grassmann coefficients of the function $E \mapsto \mathrm{e}^{\gamma^{-1}}( \pm \mathrm{i} E-\epsilon) \sum_{x \in \Lambda} \Phi_{x}^{+} \Phi_{x}^{-}$are holomorphic. Furthermore, we observe that the superfunction $\Phi \mapsto \mu_{\Lambda}^{ \pm}(\Phi) \mathrm{e}^{\gamma^{-1}( \pm \mathrm{i} E-\epsilon) \sum_{x \in \Lambda} \Phi_{x}^{+} \Phi_{x}^{-}}$belongs to $\mathscr{S}\left(\mathcal{S}^{\Lambda}, \mathscr{G}^{\Lambda}\right)$ if $\epsilon>0$ and if respectively $\pm \operatorname{Im} E \geq 0$. Finally, since by assumption $F_{0} \in L^{1}(\mathcal{S}, \mathscr{G})$, we have that

$$
\begin{aligned}
& \sup _{\sigma, \sigma^{\prime}} \int \mathrm{d} \phi_{\Lambda}\left|\int \mathrm{d} \psi_{\Lambda} \mu_{\Lambda}^{ \pm}(\Phi) \mathrm{e}^{\gamma^{-1}( \pm \mathrm{i} E-\epsilon) \sum_{x \in \Lambda} \Phi_{x}^{+} \Phi_{x}^{-}} \psi_{x, \sigma}^{-} \psi_{y, \sigma^{\prime}}^{+} F_{0}^{\Lambda}(\Phi)\right| \\
& \leq\left\|\Phi \mapsto \mu_{\Lambda}^{ \pm}(\Phi) \mathrm{e}^{\gamma^{-1}( \pm \mathrm{i} E-\epsilon) \sum_{x \in \Lambda} \Phi_{x}^{+} \Phi_{x}^{-}}\right\|_{L^{\infty}\left(\mathcal{S}^{\Lambda}, \mathscr{G} \Lambda\right)}\left\|F_{0}^{\Lambda}\right\|_{L^{1}\left(\mathcal{S}^{\Lambda}, \mathscr{G} \Lambda\right)}
\end{aligned}
$$

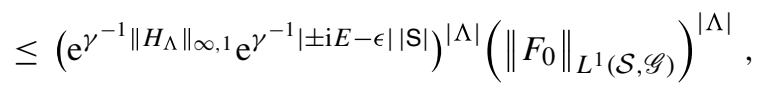

where we used the properties of the Grassmann norms and the bound (2.59). Hence, at fixed $\Lambda$ and $\epsilon$ the first line in (2.63) is uniformly bounded on compacts respectively in the domains $\pm \operatorname{Im} E \geq 0$, therefore $\mathbb{G}_{\Lambda}(x, y ; E \pm \mathrm{i} \epsilon)$ is analytic respectively in $\pm \operatorname{Im} E>0$ as claimed.

To conclude, we prove claim (ii) by applying super Plancherel identity (see Proposition 2.8) to the r.h.s. of (2.51), and use the fact that

$$
\frac{\partial}{\partial \eta_{x}^{+}} \frac{\partial}{\partial \eta_{y}^{-}} \widehat{F}_{0}^{\Lambda}(\xi)=\int \mathrm{d} \Phi_{\Lambda} \mathrm{e}^{-\mathrm{i} \sum_{x^{\prime} \in \Lambda}\left(\xi_{x^{\prime}}^{+} \Phi_{x^{\prime}}^{-}+\Phi_{x^{\prime}}^{+} \xi_{x^{\prime}}^{-}\right)} \psi_{x}^{-} \psi_{y}^{+} F_{0}^{\Lambda}(\Phi) \text {. }
$$


Remark 2.15 Our proof of the SUSY representation relies on $v$ having finite moments (H1). This hypothesis can be weakened by use of, e.g., supersymmetric polar coordinates [22]. In such a case, the SUSY representation could possibly involve a more complicated expression than $\hat{v}\left(\Phi^{+} \Phi^{-}\right)$.

With stronger assumptions on $v$, we can analytically extend the SUSY integral and hence $\mathbb{G}_{\Lambda}(x, y ; E \pm \mathrm{i} \epsilon)$ in the variable $E$.

Lemma 2.16 (Analytic continuation) Let $\Lambda \subset \mathbb{Z}^{\mathrm{D}}$ be finite and $\epsilon \geq 0$. Assume (H1) and that $F_{\beta} \in L^{1}(\mathcal{S}, \mathscr{G})$ for some $\beta>0$. Then $\mathbb{G}_{\Lambda}(x, y ; E+\mathrm{i} \epsilon)$ and $\mathbb{G}_{\Lambda}(x, y ; E-$ $\mathrm{i} \epsilon)$ can be continued to functions that are analytic respectively in $\operatorname{Im} E>-\beta$ and $\operatorname{Im} E<\beta$ and continuous respectively in $\operatorname{Im} E \geq-\beta$ and $\operatorname{Im} E \leq \beta$.

Proof Thanks to (H1) the representation in (2.51) holds. As was done above, we prove that $\mathbb{G}_{\Lambda}(x, y ; E \pm \mathrm{i} \epsilon)$ has an analytic extension by showing that the r.h.s. of (2.51) is analytic in the relevant domain. This is again achieved by standard application of the Fubini-Tonelli and Morera's theorem. It suffices to notice that for any $E \in \mathbb{C}$ such that $0 \leq \mp \operatorname{Im} E \leq \beta$, we respectively have

$$
\begin{aligned}
& \left\|F_{ \pm \mathrm{i} E-\epsilon}(\Phi)\right\|=\left\|\mathrm{e}^{\gamma^{-1}( \pm \mathrm{i} \operatorname{Re} E-(\epsilon+\beta \pm \operatorname{Im} E)) \Phi^{+} \Phi^{-}} F_{\beta}(\Phi)\right\| \\
& \leq \mathrm{e}^{\gamma^{-1}| \pm \mathrm{i} E-(\epsilon+\beta)||\mathrm{S}|}\left\|F_{\beta}(\Phi)\right\|
\end{aligned}
$$

where we used that $\epsilon+\beta \pm \operatorname{Im} E \geq 0$ respectively. Accordingly,

$$
\begin{aligned}
& \sup _{\sigma, \sigma^{\prime}} \int \mathrm{d} \phi_{\Lambda}\left|\int \mathrm{d} \psi_{\Lambda} \mu_{\Lambda}^{ \pm}(\Phi) \psi_{x, \sigma}^{-} \psi_{y, \sigma^{\prime}}^{+} F_{ \pm \mathrm{i} E-\epsilon}^{\Lambda}(\Phi)\right|
\end{aligned}
$$

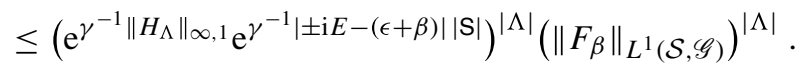

Thus, at fixed $\Lambda$ and $\epsilon \geq 0$ the first line in (2.66) is uniformly bounded on compacts respectively in the strips $0<\mp \operatorname{Im} E<\beta$ and the claims follow.

We will henceforth assume that $\mathbb{G}_{\Lambda}(x, y ; E \pm \mathrm{i} \epsilon)$ is the analytic extension if possible.

The main technical difficulty in the analysis of the SUSY integrals in Eq. (2.51) and Eq. (2.53) is to obtain estimates that are uniform in the volume. We will achieve this goal by means of SUSY cluster expansions, which we discuss in detail in the next two sections.

\section{SUSY Cluster Expansion at Strong Disorder}

In this section, we prove the exponential decay of the disorder-averaged Green's function (Theorem 3.2) and we establish the analiticity of the LDOS (Corollary 3.4) at strong disorder and arbitrary energies. The analysis of the direct SUSY integral is based on the SUSY cluster expansion presented in Proposition 3.9. The proof of the theorem is then completed by means of tree estimates together with some suitable 
bounds on the norm of the superfunctions to be integrated. The latter bounds can be achieved under some reasonable assumptions on the disorder distribution that we anticipated in (H2), see the Introduction. In order to make the assumption precise, we need the following definition.

Definition 3.1 (Integrable Multiplicative Bounds) Let $f=f(\Phi)$ be a function of a supervector $\Phi=(\phi, \psi) \in \mathcal{S}$. We say that $f$ satisfies integrable multiplicative bounds (IMB) if for some $K \geq 0, M \geq 1$ and $p \geq 0$ the following holds true:

$$
\int \mathrm{d} \phi\left\|\left(\prod_{\varepsilon= \pm} \prod_{\sigma \in \mathrm{S}}\left(\phi_{\sigma}^{\varepsilon}\right)^{n_{\sigma}^{\varepsilon}}\right) f(\Phi)\right\| \leq K M^{n}(n !)^{p},
$$

for all $\left\{n_{\sigma}^{\varepsilon}\right\} \in \mathbb{N}^{S \times\{ \pm\}}$, having set $n:=\sum_{\varepsilon, \sigma} n_{\sigma}^{\varepsilon}$.

We can then state our assumption, which depends on a parameter $\tau \geq 0$ :

$(\mathrm{H} 2-\mathrm{I})_{\tau}: \quad$ For any $\tau^{\prime} \in[0, \tau]$ the superfunction $F_{\tau^{\prime} \gamma}(\Phi)=\mathrm{e}^{\tau^{\prime} \Phi^{+} \Phi^{-}} \hat{v}\left(\Phi^{+} \Phi^{-}\right)$ satisfies IMB for some $K, M$ and $p$ depending on $\tau$.

Notice that requiring IMB over $[0, \tau]$ with $K, M$ and $p$ depending on $\tau$ is very natural, see the discussion in the appendix. We believe that our analysis could be extended to the case of weakly positively correlated disorder if we make assumptions on the decay of $v$ that are stronger than $(\mathrm{H} 2-\mathrm{I})_{\tau}$, but still applicable to Gaussian disorder.

We can now state the main results of this section.

Theorem 3.2 Let $E \in \mathbb{R}$ and $\theta \in[0,1)$. Assume that (H1) and (H2-I) $)_{\tau}$ hold for some $\tau \geq 0$. Furthermore, assume that the matrix elements of the Hamiltonian decay as

$$
\sum_{\sigma, \sigma^{\prime} \in \mathrm{S}}\left|\left(H_{x, y}\right)_{\sigma, \sigma^{\prime}}\right| \leq \mathcal{C} \mathrm{e}^{-\alpha|x-y|}
$$

for some $\mathcal{C}, \alpha>0$. Then, there exists a constant $C_{K, M, p, \theta}=C_{K, M, p, \theta}(E)>0$, depending also on $\mathcal{C}$ and $\alpha$, such that, if $\gamma \geq C_{K, M, p, \theta}$, we have that for $z_{ \pm}=E \mp \mathrm{i} \beta$ with $0 \leq \beta \leq \tau \gamma$

$$
\sup _{\sigma, \sigma^{\prime}}\left|\left(\mathbb{G}_{\Lambda}\left(x, y ; z_{ \pm} \pm \mathrm{i} \epsilon\right)\right)_{\sigma, \sigma^{\prime}}\right| \leq \gamma^{-2+\delta_{x, y}}\left(C_{K, M, p, \theta}\right)^{2-\delta_{x, y}} \mathrm{e}^{-\theta \alpha|x-y|},
$$

uniformly in $\Lambda \subset \mathbb{Z}^{\mathrm{D}}$ and $0 \leq \epsilon \leq 1$. The constant $C_{K, M, p, \theta}$ grows as $|E|^{|\mathrm{S}| / 1+|\mathrm{S}|}$ at large $|E|$.

Remark 3.3 In particular, the theorem holds for $E \in \sigma(H)$ and $\epsilon=0$, which is the most interesting range of parameters. As soon as $E \pm \mathrm{i} \epsilon$ is sufficiently away from the unperturbed spectrum, the result in Theorem $4 . .2$ is better suited and gives uniform estimates as $|E|$ or $\epsilon$ become large.

Corollary 3.4 Let $\mathcal{B} \subset \mathbb{R}$ non-empty and bounded. Assume that $(\mathrm{HI})$ and $(\mathrm{H} 2-\mathrm{I})_{\tau}$ hold for some $\tau>0$. Furthermore, assume that the Hamiltonian satisfies (3.2) for 
some $\mathcal{C}, \alpha>0$. Then, there exists a constant $C_{K, M, p}=C_{K, M, p}(\mathcal{B})>0$, depending also on $\mathcal{C}$ and $\alpha$, such that, if $\gamma \geq C_{K, M, p}, \rho(E)$ can be extended to an analytic function in $(\operatorname{Re} E, \operatorname{Im} E) \in \mathcal{B} \times(-\tau \gamma, \tau \gamma)$.

Proof Our proof is a rephrasing of the one provided in [15]. For the sake of brevity, set $\mathbb{G}_{\Lambda}(z):=\mathbb{G}_{\Lambda}(0,0 ; z) \in \mathbb{C}^{S \times S}$ and $\beta:=\tau \gamma$. The objective is to prove the analyticity of

$$
\lim _{\epsilon \rightarrow 0^{+}} \lim _{\Lambda \nearrow \mathbb{Z}^{\mathrm{D}}}\left(\operatorname{Tr}_{S} \mathbb{G}_{\Lambda}(z+\mathrm{i} \epsilon)-\operatorname{Tr}_{\mathbf{S}} \mathbb{G}_{\Lambda}(z-\mathrm{i} \epsilon)\right)
$$

for $z \in \mathcal{B} \times(-\beta, \beta) \subset \mathbb{C}$. Since $F_{\beta}$ satisfies IMB, the hypothesis of Lemma 2.16 is satisfied and hence for any fixed $\Lambda \subset \mathbb{Z}^{\mathrm{D}}$ the function $\operatorname{Tr}_{\mathrm{S}} \mathbb{G}_{\Lambda}(z \pm \mathrm{i} \epsilon)$ is analytic in $z \in \mathcal{B} \times(-\beta, \beta)$ and continuous in $z \in \mathcal{B} \times[-\beta, \beta]$. The hypotheses of Theorem 3.2 are also satisfied: we use the theorem with $\theta=0$, and with $\operatorname{Re} z \in \mathcal{B}$. Define $C_{K, M, p}:=\sup _{E \in \mathcal{B}} C_{K, M, p, \theta=0}(E), C_{K, M, p, \theta=0}(E)$ being the constant in Theorem 3.2. As a consequence of the theorem, if $\gamma \geq C_{K, M, p}$ then uniformly in $z \in \mathcal{B} \times(-\beta, \beta), 0 \leq \epsilon \leq 1$ and $\Lambda \subset \mathbb{Z}^{\mathrm{D}}$, the quantities $\operatorname{Tr}_{\mathrm{S}} \mathbb{G}_{\Lambda}(z+\mathrm{i} \epsilon)$ and $\operatorname{Tr}_{S} \mathbb{G}_{\Lambda}(z-\mathrm{i} \epsilon)$ are bounded. Since by the second resolvent identity the limit

$$
\lim _{\Lambda \nearrow \mathbb{Z}^{\mathrm{D}}} \operatorname{Tr}_{\mathbf{S}} \mathbb{G}_{\Lambda}(z \pm \mathrm{i} \epsilon)=\operatorname{Tr}_{\mathbf{S}} \mathbb{G}_{\mathbb{Z}^{\mathrm{D}}}(z \pm \mathrm{i} \epsilon)
$$

exists for any $\epsilon>0$, we can apply the Vitali-Porter theorem and obtain that the convergence is uniform with $\operatorname{Tr}_{S} \mathbb{G}_{\mathbb{Z}^{\mathrm{D}}}(z \pm \mathrm{i} \epsilon)$ analytic in $z \in \mathcal{B} \times(-\beta, \beta), \epsilon>0$ and continuous in $\epsilon \rightarrow 0^{+}$. Therefore, both

$$
\lim _{\epsilon \rightarrow 0^{+}} \operatorname{Tr}_{\mathbf{S}} \mathbb{G}_{\mathbb{Z}^{\mathrm{D}}}(z+\mathrm{i} \epsilon) \quad \lim _{\epsilon \rightarrow 0^{+}} \operatorname{Tr}_{\mathbf{S}} \mathbb{G}_{\mathbb{Z}^{\mathrm{D}}}(z-\mathrm{i} \epsilon)
$$

exist and are analytic in $z \in \mathcal{B} \times(-\beta, \beta)$, hence the claim.

Remark 3.5 In [57] they prove directly that the limit

$$
\lim _{\epsilon \rightarrow 0^{+}} \lim _{\Lambda \nearrow \mathbb{Z}^{\mathrm{D}}} \mathbb{G}_{\Lambda}(x, y ; E \pm \mathrm{i} \epsilon)
$$

exists provided that either $\gamma$ or $E$ is large. We point out that the expansion presented in Proposition 3.9 and the estimates in the proof of Theorem 3.2 allow us to prove that uniformly in $0 \leq \epsilon \leq 1,\left\{\left(\mathbb{G}_{\Lambda}(x, y ; E \pm \mathrm{i} \epsilon)\right)_{\sigma, \sigma^{\prime}}\right\}_{\Lambda}$ is a Cauchy sequence in $\Lambda$, at any fixed $x, y \in \mathbb{Z}^{\mathrm{D}}, \sigma, \sigma^{\prime} \in \mathrm{S}$ and $E \in \mathbb{R}$.

The SUSY cluster expansion presented in Proposition 3.9 is crucially based on the following important result in statistical mechanics.

Theorem 3.6 (Battle-Brydges-Federbush representation) For any $\mathrm{X} \subset \Lambda$ let $V_{\mathrm{X}}:=$ $1 / 2 \sum_{x, y \in \mathrm{X}} v_{x, y}$, where $v_{x, y}=v_{y, x}$ is an even element of a Grassmann algebra. The following representation holds true:

$$
\mathrm{e}^{V_{\mathrm{X}}}=\sum_{\Pi \in \operatorname{Part}(\mathrm{X})} \prod_{\mathrm{Y} \in \Pi} K(\mathrm{Y}),
$$


where the sum is over the partitions of $\mathrm{X}$ and where $K(\mathrm{Y})=\mathrm{e}^{V_{\mathrm{Y}}}$ if $|\mathrm{Y}|=1$, otherwise

$$
K(\mathrm{Y})=\sum_{\mathrm{T} \text { on } \mathrm{Y}}\left(\prod_{\{x, y\} \in \mathrm{T}} v_{x, y}\right) \int \mathrm{d} p_{\mathrm{T}}(s) \mathrm{e}^{V_{\mathrm{Y}}(s)}
$$

For $s=\left(s_{x, y}\right) \in[0,1]^{\mathcal{P}(\mathrm{Y})}, \mathcal{P}(\mathrm{Y})$ being the set of unordered pairs in $\mathrm{Y}$, we have defined

$$
V_{\mathrm{Y}}(s):=\frac{1}{2} \sum_{x, y \in \mathrm{Y}} s_{x, y} v_{x, y}
$$

and denoted by $\mathrm{d} p_{\mathrm{T}}$ a probability measure supported on $s \in[0,1]^{\mathcal{P}(\mathrm{Y})}$ such that $V_{\mathrm{Y}}(s)$ is a convex decoupling of $V_{\mathrm{Y}}$, that is, it is a convex linear combination of quantities of the form $\sum_{\mathrm{Y}^{\prime} \in \Pi} V_{\mathrm{Y}^{\prime}}$, with $\Pi$ being a partition of $\mathrm{Y}$.

The proof of this statement can be found in [16, Appendix B]. See also [1, 7, 17, 46] for other proofs of the Battle-Brydges-Federbush formula or generalizations of it. Technically speaking, the proof in [16] is stated for the case of $v_{x, y} \in \mathbb{C}$. However, since both sides of the Battle-Brydges-Federbush formula are analytic in $v_{x, y}$, the identity holds also when $v_{x, y}$ is an even element of a Grassmann algebra or more generally of a commutative Banach algebra, see [1] for such generalizations.

Remark 3.7 We say that $V_{\mathrm{X}}$ is stable if the real part of $\left(V_{\mathrm{X}}\right)_{\emptyset}$ is non-positive. If $V_{\mathrm{X}}$ is stable also its convex decouplings are stable: this property is crucial in order to have well-defined integrals. All the cases we consider below have this stability property provided that $\epsilon \geq 0$. For example, $V_{\mathrm{X}}=\mathrm{i} \sum_{x, y \in \mathrm{X}} \Phi_{x}^{+}\left(H_{x, y}+\right.$ $\left.(E+\mathrm{i} \epsilon) \delta_{x, y}\right) \Phi_{y}^{-}$and $\hat{V}_{\mathrm{X}}=\mathrm{i} \sum_{x, y \in \mathrm{X}} \xi_{x}^{+} C_{x, y}^{E+\mathrm{i} \epsilon} \xi_{y}^{-}$with $\epsilon \geq 0$ are stable, in fact, $\operatorname{Re}\left(V_{\mathrm{X}}\right)_{\emptyset}=\operatorname{Re} \mathrm{i} \sum_{x, y \in \mathrm{X}} \phi_{x}^{+}\left(H_{x, y}+(E+\mathrm{i} \epsilon) \delta_{x, y}\right) \phi_{y}^{-} \leq 0$ and $\operatorname{Re}\left(\hat{V}_{\mathrm{X}}\right)_{\emptyset}=$ $\operatorname{Re} \mathrm{i} \sum_{x, y \in \mathrm{X}} \kappa_{x}^{+} C_{x, y}^{E+\mathrm{i} \epsilon} \kappa_{y}^{-} \leq 0$. Incidentally, notice that this particular $V_{\mathrm{X}}(s)$ is stable for any $s \in[0,1]^{\mathcal{P}(\mathrm{Y})}$.

Remark 3.8 With abuse of notation we shall introduce the "empty tree" and denote it by $\emptyset$, as the only tree-graph on a set $\mathrm{Y}$ with $|\mathrm{Y}|=1$. Furthermore, $\int \mathrm{d} p_{\mathrm{T}=\emptyset}(s)$ is a complicated way to denote the multiplication identity. Thus, if $|\mathrm{Y}|=1$ we define

$$
\mathrm{e}^{V_{\mathrm{Y}}}=: \sum_{\mathrm{T} \text { on } \mathrm{Y}}\left(\prod_{\{x, y\} \in \mathrm{T}} v_{x, y}\right) \int \mathrm{d} p_{\mathrm{T}}(s) \mathrm{e}^{V_{\mathrm{Y}}(s)} .
$$

We apply the BBF representation to the super Gibbs' weights $\mu_{\Lambda}^{ \pm}(\Phi)$. The expansion is well suited for our purposes because it exploits the smallness of $\gamma^{-1}$ : it makes the fact that $\mu_{\Lambda}^{ \pm}(\Phi)$ is close to one quantitative precise. The expansion can also be considered as an improvement of the simple Taylor expansion of $\mu_{\Lambda}^{ \pm}(\Phi)$. 
Proposition 3.9 Let $E \in \mathbb{R}$. Assume that (H1) and (H2-I) $)_{\tau}$ hold for some $\tau \geq 0$. Then, the following representation holds true for any $\epsilon \geq 0$ and for $z_{ \pm}:=E \mp \mathrm{i} \beta$ with $0 \leq \beta \leq \tau \gamma$ :

$$
\begin{aligned}
\mathbb{G}_{\Lambda}\left(x, y ; z_{ \pm} \pm \mathrm{i} \epsilon\right)= & \left( \pm \mathrm{i} \gamma^{-1}\right)^{2-\delta_{x, y}} \sum_{N=0}^{\left|\Lambda^{\prime}\right|}\left( \pm \mathrm{i} \gamma^{-1}\right)^{N} g_{N}\left(x, y ; z_{ \pm} \pm \mathrm{i} \epsilon\right) \\
g_{N}\left(x, y ; z_{ \pm} \pm \mathrm{i} \epsilon\right):= & \frac{1}{N !} \sum_{\substack{\mathrm{T} \text { on } \\
\left\{1, \ldots, N+2-\delta_{x, y}\right\}}} \sum_{\substack{x_{1}, \ldots, x_{N} \in \Lambda^{\prime} \\
\text { distinct }}} \int \mathrm{d} p_{\mathrm{T}}(s) \\
& \int \mathrm{d} \mu_{\mathrm{Y}}^{ \pm}(\Phi, s)\left(\prod_{\{i, j\} \in \mathrm{T}} v_{x_{i}, x_{j}}(\Phi)\right) F_{ \pm \mathrm{i} E-\epsilon}^{\mathrm{Y}}(\Phi) \psi_{x}^{-} \psi_{y}^{+},
\end{aligned}
$$

where we have defined $\mathrm{d} \mu_{\mathrm{Y}}^{ \pm}(\Phi, s):=\mathrm{d} \Phi_{\mathrm{Y}} \mu_{\mathrm{Y}}^{ \pm}(\Phi, s)$ and

$$
\begin{aligned}
& \mu_{\mathrm{Y}}^{ \pm}(\Phi, s):=\mathrm{e}^{ \pm \frac{\mathrm{i}}{2} \gamma^{-1} \sum_{x, y \in \mathrm{Y}} s_{x, y} v_{x, y}(\Phi)}, \\
& v_{x, y}(\Phi):=-\Phi_{x}^{+} H_{x, y} \Phi_{y}^{-}-\Phi_{y}^{+} H_{y, x} \Phi_{x}^{-} .
\end{aligned}
$$

with $s=\left(s_{x, y}\right) \in[0,1]^{\mathcal{P}(\mathrm{Y})}$ and with $\mathrm{d} p_{\mathrm{T}}(s)$ being a probability measure. Furthermore, we have set $x_{N+1}:=x$ and $x_{N+2}:=y, \mathrm{Y}:=\left\{x_{1}, \ldots, x_{N+2}\right\}$ and $\Lambda^{\prime}:=\Lambda \backslash\{x, y\}$.

Remark 3.10 It is important to notice that the expansion of $\mathbb{G}_{\Lambda}$ is in terms of connected graphs, specifically tree-graphs, while usually one would expect an expansion in forests [1]. This is ultimately a consequence of supersymmetry which bypasses the need for logarithms. This remarkable observation goes back at least as far as [15].

Remark 3.11 Notice that only the empty-tree term contributes to the quantity $g_{0}\left(x, x ; z_{ \pm} \pm \mathrm{i} \epsilon\right)$, for which definition (3.10) applies.

Proof The superfunction $F_{\beta}$ is well-defined thanks to (H1) and satisfies IMB for any $0 \leq \beta \leq \tau \gamma$ thanks to $(\mathrm{H} 2-\mathrm{I})_{\tau}$. In particular, $F_{\beta} \in L^{1}(\mathcal{S}, \mathscr{G})$ so that by Lemma 2.16 we can (analytically) continue the direct SUSY integral in (2.51) as $E \rightarrow z_{ \pm}$, $\epsilon \geq 0$. Since $v_{x, y}(\Phi)$ are even elements of a Grassmann algebra, we apply Theorem 3.6 to $\mu_{\Lambda}^{ \pm}=: \mathrm{e}^{V_{\Lambda}^{ \pm}}$and after simple manipulations we obtain the following polymer expansion:

$$
\int \mathrm{d} \mu_{\Lambda}^{ \pm}(\Phi) F_{ \pm \mathrm{i} z_{ \pm}-\epsilon}^{\Lambda}(\Phi) \psi_{x}^{-} \psi_{y}^{+}=\sum_{\Pi \in \operatorname{Part}(\Lambda)} \prod_{\mathrm{Y} \in \Pi} K(\mathrm{Y})
$$

where

$$
\begin{aligned}
K(\mathrm{Y})= & \sum_{\mathrm{T} \text { on } \mathrm{Y}} \int \mathrm{d} p_{\mathrm{T}}(s) \int \mathrm{d} \mu_{\mathrm{Y}}^{ \pm}(\Phi, s) \\
& \left(\prod_{\left\{x^{\prime}, y^{\prime}\right\} \in \mathrm{T}} \pm \mathrm{i} \gamma^{-1} v_{x^{\prime}, y^{\prime}}(\Phi)\right) F_{ \pm \mathrm{i}_{z^{-}}-\epsilon}^{\mathrm{Y}}(\Phi)\left(\psi_{x}^{-}\right)^{\mathbf{1}_{\mathrm{Y}}(x)}\left(\psi_{y}^{+}\right)^{\mathbf{1}_{\mathrm{Y}}(y)},
\end{aligned}
$$

$\mathbf{1}_{\mathrm{Y}}(\cdot)$ denoting the indicator function of $\mathrm{Y}$ and $\left(\psi_{x, \sigma}^{\varepsilon}\right)^{0}=1$ by convention. The final expression in (3.15) has been obtained by swapping the integration over the 
superfields $\Phi$ with the integration over $s$, which we can do since uniformly in $s$ the superintegral is bounded. This swap is mainly a matter of taste. Notice that by the BBF formula $\mathrm{d} p_{\mathrm{T}}(s)$ is supported on $s$ such that $V_{\mathrm{X}}^{ \pm}(s)$ are convex decouplings of $V_{\mathrm{X}}^{ \pm}$. However, since $V_{\mathrm{X}}^{ \pm}(s)$ are stable for any $s \in[0,1]^{\mathcal{P}(\mathrm{X})}$, this information is superfluous in this special case.

We shall now make the expansion in (3.14) simpler by symmetry considerations. To begin, we notice that $\mu_{\mathrm{Y}}^{ \pm}(\cdot, s), v_{x, y}$ and $F_{ \pm \mathrm{i} z_{ \pm}-\epsilon}^{\mathrm{Y}}$ are even and supersymmetric, see also discussion before Proposition 2.14. Thus, by parity $K(\mathrm{Y})=0$ unless $\mathrm{Y} \cap\{x, y\}$ is either $\{x, y\}$ or $\emptyset$. On the other hand, if $\mathrm{Y} \cap\{x, y\}=\emptyset$, the superfunction to be integrated with respect to $\int \mathrm{d} \Phi_{\mathrm{Y}}$ is supersymmetric. If we show that such integrand satisfies the hypotheses of the SUSY localization formula, then $K(\mathrm{Y})=\delta_{1,|\mathrm{Y}|}$ whenever $\mathrm{Y} \cap\{x, y\}=\emptyset$, since in fact $\mu_{\mathrm{Y}}^{ \pm}(0, s)=F_{ \pm \mathrm{i} z_{ \pm}-\epsilon}^{\mathrm{Y}}(0)=1$ and $v_{x, y}(0)=0$. Thus, we are left with proving that for any $(x, \sigma, \varepsilon) \in \mathbf{Y}^{ \pm} \times\{ \pm\}$the superfunction

$$
\Phi \mapsto \frac{\psi_{x, \sigma}^{\varepsilon}}{\left(1+\left|\phi_{x, \sigma}^{\varepsilon}\right|\right)} \frac{\partial}{\partial \phi_{x, \sigma}^{\varepsilon}}\left(\mu_{\mathrm{Y}}^{ \pm}(\Phi, s)\left(\prod_{\left\{x^{\prime}, y^{\prime}\right\} \in \mathrm{T}} v_{x^{\prime}, y^{\prime}}(\Phi)\right) F_{ \pm \mathrm{i} z_{ \pm}-\epsilon}^{\mathrm{Y}}(\Phi)\right)
$$

is in $L^{1}\left(\mathcal{S}^{\mathrm{Y}}, \mathscr{G}^{\mathrm{Y}}\right)$, see Proposition 2.12. When the derivative acts on $\mu_{\mathrm{Y}}^{ \pm}(\cdot, s)$ or $v_{x^{\prime}, y^{\prime}}$ no dangerous terms are generated and the integrability follows once more because $F_{\beta}$ satisfies IMB. If, instead, the derivative acts on $F_{ \pm \mathrm{i} z_{ \pm}-\epsilon}^{\mathrm{Y}}$, since $F_{z}$ is invariant under $U(1)^{\times \mathrm{S}}$ fermionic transformations, Lemma 2.13 implies the identity

$$
\frac{\psi_{\sigma}^{\varepsilon}}{\left(1+\left|\phi_{\sigma}^{\varepsilon}\right|\right)} \frac{\partial}{\partial \phi_{\sigma}^{\varepsilon}} F_{z}(\Phi)=-\varepsilon \frac{\phi_{\sigma}^{-\varepsilon}}{\left(1+\left|\phi_{\sigma}^{\varepsilon}\right|\right)} \frac{\partial}{\partial \psi_{\sigma}^{-\varepsilon}} F_{z}(\Phi),
$$

and hence integrability of (3.16) because $F_{\beta}$ satisfies IMB.

The previous considerations imply that the sum in (3.14) is really just

$$
\begin{aligned}
\sum_{\mathrm{Y} \supset\{x, y\}} K(\mathrm{Y}) & =\sum_{N=0}^{\left|\Lambda^{\prime}\right|} \sum_{\substack{\mathrm{Y} \supset\{x, y\} \\
|\mathrm{Y}|=N}} K(\mathrm{Y}) \\
& =\sum_{N=0}^{\left|\Lambda^{\prime}\right|} \frac{1}{N !} \sum_{\substack{x_{1}, \ldots, x_{N} \in \Lambda^{\prime} \\
\text { distinct }}} K\left(\left\{x_{1}, \ldots, x_{N}, x, y\right\}\right) .
\end{aligned}
$$

The $1 / N$ ! factor is needed because each set $Y$ is counted that many times in the sum over the distinct points $x_{1}, \ldots, x_{N}$. We then set $x_{N+1}:=x, x_{N+2}:=y$ and swap the sum over distinct points with the sum over trees on $\mathrm{Y}$, the latter becoming a sum over trees on $\left\{1, \ldots, N+2-\delta_{x, y}\right\}$. Notice that if $x=y$, then the set Y contains only $N+1$ points.

Before delving into the proof of Theorem 3.2, we want to make a general remark about the strategy. In interacting fermionic systems determinant bounds provide a useful tool to control the convergence of the perturbative expansion [33, 34, 42, 43, 47]. We avoid this type of bounds and instead follow a different approach, based on a combination of the BBF formula (through Proposition 3.9) and Grassmann norms. 


\subsection{Proof of Theorem 3.2}

Thanks to $(\mathrm{H} 1)$ and $(\mathrm{H} 2-\mathrm{I})_{\tau}$ the representation in Proposition 3.9 is valid. We will prove that for some constant $\bar{C}_{K, M, p, \theta}>1$ not depending on $E$ the following bound holds true:

$$
\begin{aligned}
\sup _{\sigma, \sigma^{\prime}} \mid\left(g_{N}(x,\right. & \left.\left.y ; z_{ \pm} \pm \mathrm{i} \epsilon\right)\right)_{\sigma, \sigma^{\prime}} \mid \\
& \leq\left(1+\gamma^{-|\mathrm{S}|}(1+|E|)^{|\mathrm{S}|}\right)^{|\mathrm{Y}|}\left(\bar{C}_{K, M, p, \theta}\right)^{|\mathrm{Y}|} \alpha^{-\mathrm{D} N} \mathrm{e}^{-\theta \alpha|x-y|} .
\end{aligned}
$$

By plugging this bound into the expansion in (3.11) we see that if

$$
\gamma \geq 4 \bar{C}_{K, M, p, \theta}\left(\alpha^{-\mathrm{D}}+\alpha^{-\mathrm{D} /(1+|\mathrm{S}|)}\right)(1+|E|)^{|\mathrm{S}| /(1+|\mathrm{S}|)}
$$

the expansion is convergent and the claim of the theorem ensues. It also follows that the constant $C_{K, M, p, \theta}$ grows like $|E|^{|\mathrm{S}| /(1+|\mathrm{S}|)}$ at large $|E|$.

To prove (3.19) we proceed as follows. Let us consider a polymer $\mathrm{Y} \ni x, y$ and an oriented tree $\mathrm{T}_{\wp}$ on $\mathrm{Y}$, that is, a tree-graph whose links are oriented, $\wp$ denoting the choice of the orientations. We denote by $\ell^{+}\left(\ell^{-}\right)$the starting (ending) vertex of the oriented link $\ell \in \mathrm{T}_{\wp}$. Links have to be oriented in order to select one of the two elements in $v_{\ell}(\Phi)$, see in (3.13). In other words, in (3.13) we want to sum over oriented trees instead of trees and for each link keep one of the two terms in $v_{\ell}(\Phi)$ depending on the orientation $\wp$. We thus introduce the sequences $\underline{\sigma}=\left\{\sigma_{\ell}^{\varepsilon} \in\right.$ $\mathrm{S}\}_{\ell \in \mathrm{T}, \varepsilon= \pm}$ and $\sharp=\{\sharp \ell \in\{B, F\}\}_{\ell \in \mathrm{T}}$. Also, we set $\Phi_{B, x, \sigma}^{\varepsilon}=\phi_{x, \sigma}^{\varepsilon}$ and $\Phi_{F, x, \sigma}^{\varepsilon}=$ $\psi_{x, \sigma}^{\varepsilon}$, and we shall henceforth write $|\mathrm{Y}|$ instead of $N+2-\delta_{x, y}=|\mathrm{Y}|$. We define:

$$
\mathcal{F}_{\mathrm{T}_{\wp}, \underline{\sharp}, \underline{\sigma}}^{\mathrm{Y}}(\Phi):=\left(\prod_{\ell \in \mathrm{T}_{\wp}} \Phi_{\sharp \ell, x_{\ell^{+}}, \sigma_{\ell}^{+}}^{+} \Phi_{\sharp \ell, x_{\ell^{-}}, \sigma_{\ell}^{-}}^{-}\right) \psi_{x}^{-} \psi_{y}^{+} F_{ \pm \mathrm{i} z_{ \pm}-\epsilon}^{\mathrm{Y}}(\Phi) .
$$

All in all, we can rewrite (3.13) as

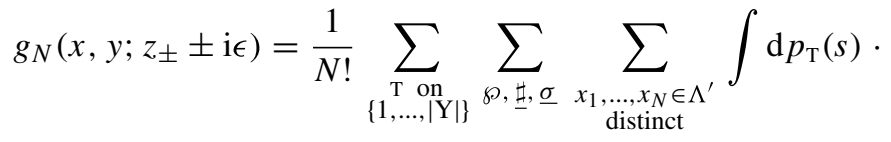

$$
\begin{aligned}
& \cdot \int \mathrm{d} \mu_{\mathrm{Y}}^{ \pm}(\Phi, s) \mathcal{F}_{\mathrm{T}_{\wp}, \sharp, \underline{\sigma}}^{\mathrm{Y}}(\Phi)\left(\prod_{\ell \in \mathrm{T}_{\wp}}\left(H_{x_{\ell^{+}}, x_{\ell^{-}}}\right)_{\sigma_{\ell}^{+}, \sigma_{\ell}^{-}}\right),
\end{aligned}
$$

which can be bounded as follows:

$$
\begin{aligned}
& \sup _{\sigma, \sigma^{\prime}}\left|\left(g_{N}\left(x, y ; z_{ \pm} \pm \mathrm{i} \epsilon\right)\right)_{\sigma, \sigma^{\prime}}\right| \leq
\end{aligned}
$$

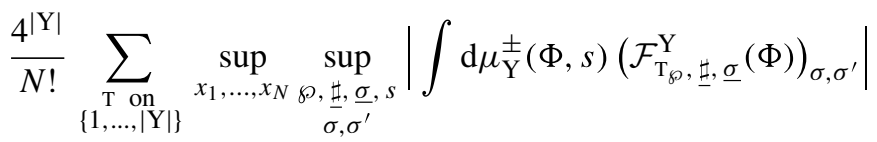

$$
\begin{aligned}
& \sup _{\wp} \sum_{\underline{\sigma}} \sum_{\substack{x_{1}, \ldots, x_{N} \in \Lambda^{\prime} \\
\text { distinct }}} \prod_{\ell \in \mathrm{T}_{\wp}}\left|\left(H_{x_{\ell^{+}}, x_{\ell^{-}}}\right)_{\hat{\sigma}_{\ell}^{+}, \hat{\sigma}_{\ell}^{-}}\right|
\end{aligned}
$$


To bound the superintegral we exploit the properties of the Grassmann norms and obtain

$$
\begin{aligned}
& \left|\int \mathrm{d} \mu_{\mathrm{Y}}^{ \pm}(\Phi, s)\left(\mathcal{F}_{\mathrm{T}_{\wp}, \underline{\sharp}, \underline{\sigma}}^{\mathrm{Y}}(\Phi)\right)_{\sigma, \sigma^{\prime}}\right| \leq \int \mathrm{d} \phi_{\mathrm{Y}}\left\|\mu_{\mathrm{Y}}^{ \pm}(\Phi, s)\right\|\left\|\left(\mathcal{F}_{\mathrm{T}_{\wp}, \underline{\sharp}, \underline{\sigma}}^{\mathrm{Y}}(\Phi)\right)_{\sigma, \sigma^{\prime}}\right\| \\
& \leq \mathrm{e}^{\gamma^{-1}\|H\|_{\infty, 1}|\mathrm{Y}|} \int \mathrm{d} \phi_{\mathrm{Y}}\left\|\left(\mathcal{F}_{\mathrm{T}_{\wp}, \underline{\sharp}, \underline{\sigma}}^{\mathrm{Y}}(\Phi)\right)_{\sigma, \sigma^{\prime}}\right\|
\end{aligned}
$$

where in the last step we used the same bound as in (2.59), noticing that $s \in$ $[0,1]^{\mathcal{P}(\mathrm{Y})}$ and that $\left\|H_{\Lambda}\right\|_{\infty, 1} \leq\|H\|_{\infty, 1}<\infty$, the latter being bounded because of (3.2).

It is clear that $\mathcal{F}_{\mathrm{T}_{\wp}, \sharp \underline{\sigma}}^{\mathrm{Y}}(\Phi)$ is a local function, i.e., it factorizes

$$
\mathcal{F}_{\mathrm{T}_{\wp}, \underline{\sharp}, \underline{\sigma}}^{\mathrm{Y}}(\Phi)= \pm \prod_{i=1}^{|\mathrm{Y}|} \mathcal{F} \frac{d_{i}}{x, y}\left(\Phi_{x_{i}}\right)
$$

having set:

$$
\mathcal{F}_{x, y} \frac{d}{}\left(\Phi_{x^{\prime}}\right):=\left(\prod_{\varepsilon, \sharp, \sigma}\left(\Phi_{\sharp, x^{\prime}, \sigma}^{\varepsilon}\right)^{d_{\sharp, \sigma}^{\varepsilon}}\right)\left(\psi_{x}^{-}\right)^{\delta_{x^{\prime}, x}}\left(\psi_{y}^{+}\right)^{\delta_{x^{\prime}, y}} F_{ \pm \mathrm{i} z_{ \pm}-\epsilon}\left(\Phi_{x^{\prime}}\right),
$$

together with the sequence $\underline{d}_{i}=\left\{\left(d_{i}\right)_{\sharp, \sigma}^{\varepsilon}\right\}_{\varepsilon= \pm, \sigma \in \mathrm{S}, \sharp=B, F}$, where $\left(d_{i}\right)_{\sharp, \sigma}^{\varepsilon}$ is defined by

$$
\left(d_{i}\right)_{\sharp, \sigma}^{\varepsilon}:=\sum_{\ell \in \mathrm{T}_{\wp}} \delta_{i, \ell^{\varepsilon}} \delta_{\sharp, \sharp \ell} \delta_{\sigma, \sigma_{\ell}^{\varepsilon}},
$$

that is, given $\mathrm{T}_{\wp}, \underline{\sigma}$ and $\sharp \underline{\sharp}$, it counts how many times $\left(\ell^{\varepsilon}, \sharp_{\ell}, \sigma_{\ell}^{\varepsilon}\right)=(i, \sharp, \sigma)$. Notice that the sign in front of the r.h.s. in (3.25) is unimportant and thus left unspecified. For the sake of notation, we also define $d_{i, \sharp}:=\sum_{\varepsilon, \sigma}\left(d_{i}\right)_{\sharp, \sigma}^{\varepsilon}$ together with $d_{i}:=\sum_{\sharp} d_{i, \sharp}$. Since $F_{ \pm \mathrm{i} z_{ \pm}-\epsilon}(\Phi)=\mathrm{e}^{\gamma^{-1}( \pm \mathrm{i} E-\epsilon) \Phi^{+} \Phi^{-}} F_{\beta}(\Phi)$ we have, compare with (2.60):

$$
\left\|\left(\mathcal{F}_{x, y}^{\underline{d}}\left(\Phi_{x^{\prime}}\right)\right)_{\sigma, \sigma^{\prime}}\right\| \leq\left(\operatorname{Tay}_{|\mathrm{S}|} \mathrm{e}^{\gamma^{-1}|\mathrm{i} E-\epsilon||\mathrm{S}|}\right)\left\|\left(\prod_{\varepsilon, \sigma}\left(\phi_{x^{\prime}, \sigma}^{\varepsilon}\right)^{d_{B, \sigma}^{\varepsilon}}\right) F_{\beta}(\Phi)\right\| .
$$

Using that $F_{\beta}$ satisfies IMB and that $\epsilon \leq 1$ we finally obtain

$$
\begin{aligned}
\sup _{\substack{\sharp, \underline{\sharp}, \underline{\sigma}, s \\
\sigma, \sigma^{\prime}}}\left|\int \mathrm{d} \mu_{\mathrm{Y}}^{ \pm}(\Phi, s)\left(\mathcal{F}_{\mathrm{T}_{\wp}, \underline{\underline{\sigma}}}^{\mathrm{Y}}(\Phi)\right)_{\sigma, \sigma^{\prime}}\right| \leq \mathrm{e}^{\gamma^{-1}\|H\|_{\infty, 1}|\mathrm{Y}|} . \\
\cdot\left[\prod_{i=1}^{|\mathrm{Y}|}\left(\operatorname{Tay}_{|\mathrm{S}|} \mathrm{e}^{\gamma^{-1}(1+|E|)|\mathrm{S}|}\right) \sup _{\wp, \underline{\sharp}, \underline{\sigma}} \int \mathrm{d} \phi_{x_{i}}\left\|\left(\prod_{\varepsilon,}\left(\phi_{x^{\prime}}^{\varepsilon}\right)^{d_{i, B}^{\varepsilon}}\right) F_{\beta}(\Phi)\right\|\right] \\
\leq \mathrm{e}^{\gamma^{-1}\|H\|_{\infty, 1}|\mathrm{Y}|} K^{|\mathrm{Y}|}\left(\operatorname{Tay}_{|\mathrm{S}|} \mathrm{e}^{\gamma^{-1}(1+|E|)|\mathrm{S}|}\right)^{|\mathrm{Y}|}\left[\prod_{i=1}^{|\mathrm{Y}|}\left(d_{i} !\right)^{p} M^{d_{i}}\right] .
\end{aligned}
$$




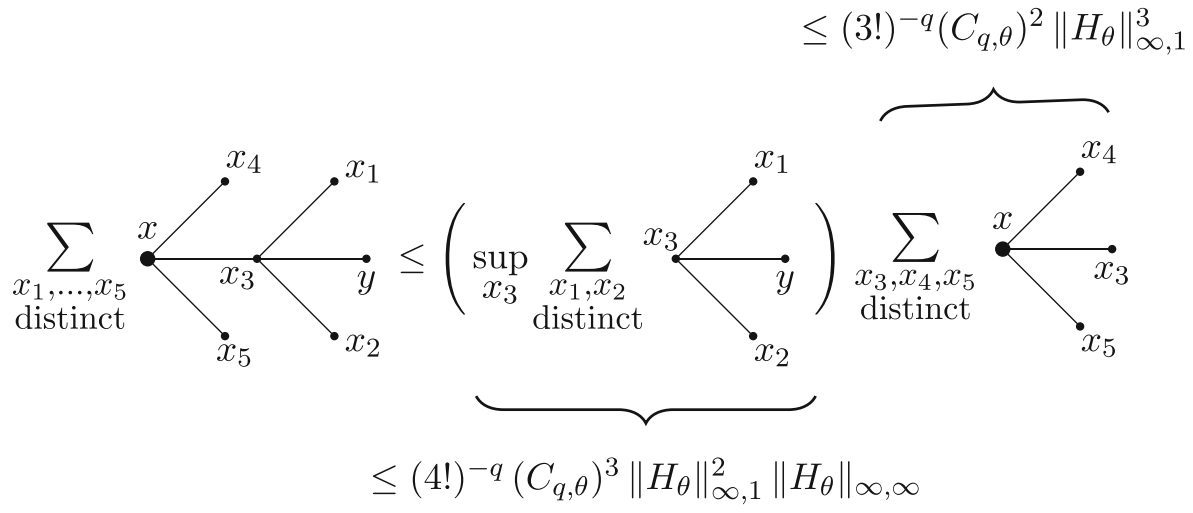

Fig. 1 Representation of the stripping procedure of (3.32). The vertices represent the spacial points and the lines the modulus of the entries of $H_{2 \theta-1}$. For simplicity, we did not represent the internal degrees of freedom. We fix a root, say $x$, and start stripping the tree from its outermost branches, those with incidence number equal to one. The estimate is then carried out iteratively

To estimate the second line in (3.23), we shall exploit the exponential decay of the hopping Hamiltonian. If we define $\left(H_{\theta}\right)_{x, y}:=\mathrm{e}^{(1+\theta) \alpha|x-y| / 2} H_{x, y}$, it follows that, see (2.50) and (3.2),

$$
\left\|H_{\theta}\right\|_{\infty, 1}=\sup _{x \in \mathbb{Z}^{\mathrm{D}}} \sum_{x^{\prime} \in \mathbb{Z}^{\mathrm{D}}} \mathrm{e}^{(1+\theta) \alpha\left|x-x^{\prime}\right| / 2} \sum_{\sigma, \sigma^{\prime} \in \mathrm{S}}\left|\left(H_{x, x^{\prime}}\right)_{\sigma, \sigma^{\prime}}\right| \leq \mathcal{C}_{\theta} \alpha^{-\mathrm{D}},
$$

where clearly $\mathcal{C}_{\theta}>\mathcal{C}$. The following quantity will be needed as well

$$
\left\|H_{\theta}\right\|_{\infty, \infty}:=\sup _{x, x^{\prime} \in \mathbb{Z}^{\mathrm{D}}} \mathrm{e}^{(1+\theta) \alpha\left|x-x^{\prime}\right| / 2} \sum_{\sigma, \sigma^{\prime} \in \mathrm{S}}\left|\left(H_{x, x^{\prime}}\right)_{\sigma, \sigma^{\prime}}\right| \leq \mathcal{C} .
$$

Standard tree-stripping estimates (see Fig. 1) based on the exponential decay of $H$ give [16]:

$$
\begin{aligned}
& \sum_{\underline{\sigma}} \sum_{\substack{x_{1}, \ldots, x_{N} \in \Lambda^{\prime} \\
\text { distinct }}} \prod_{\ell \in \mathrm{T}_{\wp}}\left|\left(H_{x_{\ell^{+}}, x_{\ell^{-}}}\right)_{\sigma_{\ell}^{+}, \sigma_{\ell}^{-}}\right| \\
& \leq \mathrm{e}^{-\theta \alpha|x-y|} \sum_{\underline{\sigma}} \sum_{\substack{x_{1}, \ldots, x_{N} \in \Lambda^{\prime} \\
\text { distinct }}} \prod_{\ell \in \mathrm{T}_{\wp}}\left|\left(\left(H_{2 \theta-1}\right)_{x_{\ell^{+}}, x_{\ell^{-}}}\right)_{\sigma_{\ell}^{+}, \sigma_{\ell}^{-}}\right| \\
& \leq \mathrm{e}^{-\theta \alpha|x-y|}\left\|H_{\theta}\right\|_{\infty, 1}^{N}\left\|H_{\theta}\right\|_{\infty, \infty}^{1-\delta_{x, y}} \prod_{i=1}^{|\mathrm{Y}|}\left(C_{q, \theta}\right)^{d_{i}-1}\left(d_{i} !\right)^{-q},
\end{aligned}
$$

where $q>0$ is a fixed parameter and where $C_{q, \theta}>1$ is a constant that depends also on $\mathrm{D}$, the dimension of the lattice. The estimate is carried out by progressively 
stripping the outer branches as shown in Fig 1. The branches that have been removed are then bounded as follows:

$$
\begin{aligned}
(d !)^{q} & \sup _{\bar{x}, \sigma} \sum_{\sigma_{1}, \ldots, \sigma_{d-1}} \sum_{\begin{array}{c}
x_{1}, \ldots, x_{d-1} \\
\text { distinct }
\end{array}} \prod_{j=1}^{d-1}\left|\left(\left(H_{2 \theta-1}\right)_{\bar{x}, x_{j}}\right)_{\sigma, \sigma_{j}}\right| \\
& \leq \mathrm{e}^{q d \ln d} \sup _{\bar{x}, \sigma} \sum_{\sigma_{1}, \ldots, \sigma_{d-1}} \sum_{\begin{array}{c}
x_{1}, \ldots, x_{d-1} \\
\text { distinct }
\end{array}} \prod_{j=1}^{d-1} \mathrm{e}^{-(1-\theta) \alpha\left|\bar{x}-x_{j}\right| / 2}\left|\left(\left(H_{\theta}\right)_{\bar{x}, x_{j}}\right)_{\sigma, \sigma_{j}}\right| \\
& \leq \mathrm{e}^{q d \ln d}\left(\sup _{x_{1}, \ldots, x_{d-1}} \prod_{j=1}^{d-1} \mathrm{e}^{-(1-\theta) \alpha\left|\bar{x}-x_{j}\right| / 2}\right)\left\|H_{\theta}\right\|_{\infty, 1}^{d-1} \\
& \leq \underbrace{\left(\operatorname { s u p } _ { d \geq 1 } ^ { \operatorname { s u n c t } } \left(\mathrm{e}^{\left.\left.2 q \ln d-(1-\theta) \alpha \Omega_{\mathrm{D}}(d-1)^{1 / \mathrm{D}}\right)\right)^{d-1}}\left\|H_{\theta}\right\|_{\infty, 1}^{d-1}\right.\right.}_{=: C_{q, \theta}^{d-1}}
\end{aligned}
$$

In the second inequality in (3.33) we used a simple bound for the factorial and we extracted an exponential weight from $H$ and pulled it out of the summation by taking the superior over all distinct points $x_{1} \neq \cdots \neq x_{d-1} \neq \bar{x}$. The latter can be computed by noticing that $\left|\left\{x \in \mathbb{Z}^{\mathrm{D}}|| x \mid=r\right\}\right| \leq \Omega_{\mathrm{D}}^{\prime} r^{\mathrm{D}-1}$, therefore $\sum_{j=1}^{d-1}\left|\bar{x}-x_{j}\right| / 2 \geq$ $\Omega_{\mathrm{D}}(d-1)^{1+1 / \mathrm{D}}$, for some constants $\Omega_{\mathrm{D}}<1$ and $\Omega_{\mathrm{D}}^{\prime}>1$. While stripping the tree, if one of the outer vertices is $y$, there is no summation and this produces simply $\left\|H_{\theta}\right\|_{\infty, \infty}$ instead of $\left\|H_{\theta}\right\|_{\infty, 1}$, see Fig. 1 . We shall remark that the factorial $\left(d_{i} !\right)^{-q}$ is gained only because we are summing over distinct points and because the matrix elements of the Hamiltonian decay exponentially.

Plugging the bounds (4.26) and (4.28) with $q=p+1$ into (4.19) and using Cayley's theorem on the number of trees with fixed coordination numbers $\left\{d_{i}\right\}_{i}$, see [16], we obtain (3.19):

$$
\begin{aligned}
\left|\left(g_{N}\left(x, y ; z_{ \pm} \pm \mathrm{i} \epsilon\right)\right)_{\sigma, \sigma^{\prime}}\right| & \leq \mathrm{e}^{-\theta \alpha|x-y|} K^{|\mathrm{Y}|} \mathrm{e}^{\mathcal{C}|\mathrm{Y}|}\left(\operatorname{Tay}_{|\mathrm{S}|} \mathrm{e}^{\gamma^{-1}(1+|E|)|\mathrm{S}|}\right)^{|\mathrm{Y}|} \mathcal{C} \\
& \cdot\left(\mathcal{C}_{\theta} \alpha^{-\mathrm{D}}\right)^{N} \sum_{\substack{\left\{d_{i}\right\}_{i}, d_{i} \geq 1 \\
\sum_{i} d_{i}=2|\mathrm{Y}|-2}} \prod_{i=1}^{|\mathrm{Y}|} \frac{\left(d_{i} !\right)^{p}\left(C_{p+1, \theta}\right)^{d_{i}-1} M^{d_{i}}}{\left(d_{i}-1\right) !\left(d_{i} !\right)^{p+1}} \\
& \leq\left(1+\gamma^{-|\mathrm{S}|}(1+|E|)^{|\mathrm{S}|}\right)^{|\mathrm{Y}|}\left(\bar{C}_{K, M, p, \theta}\right)^{|\mathrm{Y}|} \alpha^{-\mathrm{D} N} \mathrm{e}^{-\theta \alpha|x-y|}
\end{aligned}
$$

where $\bar{C}_{K, M, p, \theta}:=2 K M \mathrm{e}^{\mathcal{C}} \mathcal{C}_{\theta} \sum_{n=0}^{\infty} \frac{((n+1) !)^{p}\left(M C_{p+1, \theta}\right)^{n}}{(n !)^{p+2}}<\infty$.

\section{SUSY Cluster Expansion at Weak Disorder}

In this section, we prove the exponential decay of the disorder-averaged Green's function (Theorem 4.2), the analyticity of the LDOS (Corollary 4.4) and we establish Lifshitz-tail-type estimates for the latter (Theorem 4.5) at weak disorder and at 
energies away from the unperturbed spectrum. The analysis of the dual SUSY integral is based on the SUSY cluster expansion presented in Proposition 4.8. The proof of the theorems is again completed by means of tree estimates together with some suitable bounds on the norm of the superfunctions to be integrated. As was the case in Section 3, such bounds can be achieved under some reasonable assumptions on the disorder distribution that we anticipated in $(\mathrm{H} 2)$, see the Introduction. In order to make the assumption precise, we need the following definition.

Definition 4.1 (Integrable Derivative Bounds) Let $f=f(\Phi)$ be a function of a supervector $\Phi=(\phi, \psi)$. We say that $f$ satisfies integrable derivative bounds (IDB) if for some $K \geq 0, M \geq 1$ and $p \geq 0$ the following holds true

$$
\int \mathrm{d} \phi\left\|\left(\prod_{\varepsilon= \pm} \prod_{\sigma \in \mathrm{S}}\left(\frac{\partial}{\partial \phi_{\sigma}^{\varepsilon}}\right)^{n_{\sigma}^{\varepsilon}}\right) f(\Phi)\right\| \leq K M^{n}(n !)^{p},
$$

for all $\left\{n_{\sigma}^{\varepsilon}\right\} \in \mathbb{N}^{S \times\{ \pm\}}$, having set $n:=\sum_{\varepsilon, \sigma} n_{\sigma}^{\varepsilon}$.

We can then state our assumption, which depends on a parameter $\tau \geq 0$ :

$(\mathrm{H} 2-\mathrm{II})_{\tau}$ : For any $\tau^{\prime} \in[0, \tau]$ the superfunction $F_{\tau^{\prime} \gamma}(\Phi)=\mathrm{e}^{\tau^{\prime} \Phi^{+} \Phi^{-}} \hat{v}\left(\Phi^{+} \Phi^{-}\right)$ satisfies IDB for some $K, M$ and $p$ depending on $\tau$.

Again, requiring IDB over $[0, \tau]$ with $K, M$ and $p$ depending on $\tau$ is very natural, see the discussion in the appendix. In passing, notice that in the case of Gaussian disorder $(\mathrm{H} 2-\mathrm{II})_{\tau}$ holds for any $\tau$. We believe that our analysis could be extended to the case of weakly positively correlated disorder if we make assumptions on the regularity of $v$ that are stronger than (H2-II), but still applicable to Gaussian disorder.

We can finally state the main theorems of this section.

Theorem 4.2 Let $E \in \mathbb{R}, \theta \in[0,1)$, and set $\delta:=\operatorname{dist}(E, \sigma(H))$. Assume that $(H 1)$ and $(H 2-I I)_{\tau}$ hold form some $\tau \geq 0$. Furthermore, assume that the covariance decays as

$$
\sum_{\sigma, \sigma^{\prime} \in \mathrm{S}}\left|\left(C_{x, y}^{E \pm \mathrm{i} \epsilon}\right)_{\sigma, \sigma^{\prime}}\right| \leq \mathcal{C}_{\mathrm{D}} \frac{\mathrm{e}^{-\sqrt{\delta}|x-y|}}{1+|x-y|^{\mathrm{D}-2}}, \quad \forall \epsilon \geq 0 .
$$

Then, there exists a constant $C_{K, M, p, \theta}>0$ such that, if $\delta \geq \gamma C_{K, M, p, \theta}$, we have that for $z_{ \pm}=E \mp \mathrm{i} \beta$ with $0 \leq \beta \leq \tau \gamma$

$$
\sup _{\sigma, \sigma^{\prime}}\left|\left(\mathbb{G}_{\Lambda}\left(x, y ; z_{ \pm} \pm \mathrm{i} \epsilon\right)\right)_{\sigma, \sigma^{\prime}}\right| \leq \gamma^{-\delta_{x, y}}\left(C_{K, M, p, \theta}\right)^{2-\delta_{x, y}} \mathrm{e}^{-\theta \sqrt{\delta}|x-y|},
$$

uniformly in $\Lambda \subset \mathbb{Z}^{\mathrm{D}}$ and $\epsilon \geq 0$.

Remark 4.3 Notice that (4.2) is satisfied when $H=-\Delta_{\mathbb{Z}^{\mathrm{D}}}$ and, more generally, when $H$ exhibits quadratic dispersion at the band edges. Notice that at $x=y$ the limit $\gamma \rightarrow 0$ of the r.h.s. of (4.3) is divergent. This is due to the fact that the bound is uniform in $\epsilon \geq 0$; the meaningful way to compute the $\gamma \rightarrow 0$ limit is however at finite $\epsilon$. A bound which is uniform in $\epsilon \geq \epsilon_{0}$ for some $\epsilon_{0}>0$, and which does not 
diverge as $\gamma$ is sent to 0 can also be obtained using our methods but this is beyond our scope.

The case $\beta=0, \epsilon \rightarrow 0^{+}$gives the exponential decay of the disorder-averaged Green's function for energies up to the edges of the spectrum. On the other hand, at $\beta>0$ and $x=y$, the result implies analyticity of the LDOS in a suitable region of the complex plane.

Corollary 4.4 Assume that $(H 1)$ and $(H 2-I I)_{\tau}$ hold for some $\tau>0$. Assume that the covariance satisfies (4.2). Then, there exists a constant $C_{K, M, p}>0$ such that $\rho(E)$ can be extended to an analytic function on

$$
D_{K, M, p}(\gamma, \tau):=\left\{z \in \mathbb{C}\left|\operatorname{dist}(\operatorname{Re} z, \sigma(H))>\gamma C_{K, M, p},\right| \operatorname{Im} z \mid<\tau \gamma\right\} .
$$

The proof of this corollary is identical to that of Corollary 3.4: Lemma 2.16 gives the analyticity of $\mathbb{G}_{\Lambda}(0,0 ; E \pm \mathrm{i} \epsilon)$ while Theorem 4.2 provides the uniform bounds that allow us to extend the analyticity to $\mathbb{G}_{\mathbb{Z}^{\mathrm{D}}}\left(0,0 ; E \pm \mathrm{i}^{+}\right)$.

Our last result is as follows.

Theorem 4.5 Let $E \in \mathbb{R}$ and set $\delta:=\operatorname{dist}(E, \sigma(H))$. Assume that (H1) and (H2II) $)_{0}$ hold. Assume that the covariance satisfies (4.2) and that $C_{x, y}^{E} \in \mathbb{R}$ for any $x, y \in$ $\mathbb{Z}^{\mathrm{D}}$. Then, there exists a constant $C_{K, M, p}$ such that, if $\delta \geq \gamma C_{K, M, p}$, the following Lifshitz-tail-type estimate is satisfied

$$
\left|\rho_{\epsilon, \Lambda}(E)\right| \leq \gamma^{-1} C_{K, M, p}^{\prime} \mathrm{e}^{-\bar{C}_{K, M, p}\left(\gamma^{-1} \delta\right)^{1 / 2 p}},
$$

for some constants $C_{K, M, p}^{\prime}, \bar{C}_{K, M, p}>0$, uniformly in $\Lambda \subset \mathbb{Z}^{\mathrm{D}}$ and $\epsilon \geq 0$.

Remark 4.6 Notice that the condition $C_{x, y}^{E} \in \mathbb{R}$ is satisfied in the case $H=-\Delta_{\mathbb{Z}^{\mathrm{D}}}$ and, more generally, in systems for which the Bloch Hamiltonian is such that $\hat{H}(-k)=\overline{\hat{H}(k)}$. This property holds in many condensed-matter systems, an example of which is graphene [35].

Remark 4.7 Since the LDOS is finite, the integrated density of states IDOS can be obtained via [56]

$$
\mathcal{N}(E)=\int_{-\infty}^{E} \mathrm{~d} E^{\prime} \rho\left(E^{\prime}\right) .
$$

As it turns out, the IDOS controls the probability of having an eigenvalue of $H_{\omega, \Lambda}$ below $E[40,41]$ :

$$
\mathbb{P}\left(\left\{\sigma\left(H_{\omega, \Lambda}\right) \cap(-\infty, E) \neq \emptyset\right\}\right) \leq C|\Lambda| \mathcal{N}(E),
$$

for some constant $C>0$. A sufficiently small IDOS, e.g., as $\gamma \rightarrow 0$, allows us to prove localization via finite-volume criteria [4], see [40] for details. Integration of the Lifshitz-tail-type estimate of Theorem 4.5, up to energies below the bottom of the spectrum such that $\left(\gamma^{-1} \delta\right)^{1 / 2 p} \geq C|\ln \gamma|$, for some constant $C$, provides a sufficiently small upper bound on the IDOS. 
The proof of the theorems above is based on a dual SUSY cluster expansion. The approach is the same as the one in Proposition 3.9. This time we shall expand the super Gibbs' weight $\widehat{\mu}_{\Lambda}^{ \pm}(\xi)$ and obtain polymers connected by the covariance. The expansion is well-suited for our purposes because it exploits the smallness of $\gamma \delta^{-1}$.

Proposition 4.8 Let $E \in \mathbb{R}$. Assume that $(H 1)$ and $(H 2-I I)_{\tau}$ hold for some $\tau \geq 0$. Then, the following representation holds true for any $\epsilon \geq 0$ and for $z_{ \pm}:=E \mp \mathrm{i} \beta$ with $0 \leq \beta \leq \tau \gamma$ :

$$
\begin{aligned}
\mathbb{G}_{\Lambda}\left(x, y ; z_{ \pm} \pm \mathrm{i} \epsilon\right)= & -( \pm \mathrm{i} \gamma)^{-\delta_{x, y}} \sum_{N=0}^{\left|\Lambda^{\prime}\right|}( \pm \mathrm{i} \gamma)^{N} G_{N}\left(x, y ; z_{ \pm} \pm \mathrm{i} \epsilon\right) \\
G_{N}\left(x, y ; z_{ \pm} \pm \mathrm{i} \epsilon\right) & :=\frac{1}{N !} \sum_{\substack{\mathrm{T} \text { on } \\
\left\{1, \ldots, N+2-\delta_{x, y}\right\}}} \sum_{\substack{x_{1}, \ldots, x_{N} \in \Lambda^{\prime} \\
\text { distinct }}} \int \mathrm{d} p_{\mathrm{T}}(s) \\
& \cdot \int \mathrm{d} \widehat{\mu}_{\mathrm{Y}}^{ \pm}(\xi, s) \prod_{\{i, j\} \in \mathrm{T}} \hat{v}_{x_{i}, x_{j}}^{ \pm}(\xi) \frac{\partial}{\partial \eta_{x}^{+}} \frac{\partial}{\partial \eta_{y}^{-}} \widehat{F}_{\beta}^{\mathrm{Y}}(\xi),
\end{aligned}
$$

where we have set $\mathrm{d} \widehat{\mu}_{\mathrm{Y}}^{ \pm}(\xi, s):=\mathrm{d} \xi \widehat{\mu}_{\mathrm{Y}}^{ \pm}(\xi, s)$ with

$$
\begin{aligned}
& \widehat{\mu}_{\mathrm{Y}}^{ \pm}(\xi, s):=\mathrm{e}^{ \pm \frac{\mathrm{i}}{2} \gamma \sum_{x, y \in \mathrm{Y}} s_{x, y} \hat{v}_{x, y}^{ \pm}(\xi)} \\
& \hat{v}_{x, y}^{ \pm}(\xi):=\xi_{x}^{+} C_{x, y}^{E \pm \mathrm{i} \epsilon} \xi_{y}^{-}+\xi_{y}^{+} C_{y, x}^{E \pm \mathrm{i} \epsilon} \xi_{x}^{-} .
\end{aligned}
$$

Above, $s=\left(s_{x, y}\right) \in[0,1]^{\mathcal{P}(\mathrm{Y})}$ while $\mathrm{d} p_{\mathrm{T}}(s)$ is a probability measure with support on s such that the exponent in the super Gibbs' weights $\widehat{\mu}_{\mathrm{Y}}^{ \pm}(\xi, s)$ satisfies respectively $\operatorname{Re} \pm \mathrm{i} \sum_{x, y \in \mathrm{Y}} s_{x, y} \hat{v}_{x, y}^{ \pm}((\kappa, 0)) \leq 0$. Furthermore, we have set $x_{N+1}:=x$ and $x_{N+2}:=y, \mathrm{Y}:=\left\{x_{1}, \ldots, x_{N+2}\right\}$ and $\Lambda^{\prime}:=\Lambda \backslash\{x, y\}$.

Remark 4.9 Notice that only the empty-tree term contributes to the quantity $G_{0}\left(x, x ; z_{ \pm} \pm \mathrm{i} \epsilon\right)$.

In order to prove this SUSY cluster expansion, we need an auxiliary result that allows us to apply the SUSY localization formula in this context as well.

Lemma 4.10 Let $f \in L^{1}(\mathcal{S}, \mathscr{G})$ be even, supersymmetric and invariant under $U(1)^{\times S}$ fermionic transformations, see Lemma 2.13. Assume that the function $\Phi \mapsto$ $\psi_{\sigma}^{\varepsilon}\left(\partial / \partial \phi_{\sigma}^{\varepsilon}\right) f(\Phi)$ is in $L^{1}(\mathcal{S}, \mathscr{G})$ for any $\varepsilon, \sigma$. Then

$$
\widehat{f}(\xi)=\int \mathrm{d} \Phi \mathrm{e}^{-\mathrm{i} \xi^{+} \Phi^{-}-\mathrm{i} \Phi^{+} \xi^{-}} f(\Phi)
$$

is even, satisfies

$$
\eta_{\sigma}^{\varepsilon} \frac{\partial}{\partial \kappa_{\sigma}^{\varepsilon}} \widehat{f}(\xi)=-\varepsilon \kappa_{\sigma}^{-\varepsilon} \frac{\partial}{\partial \eta_{\sigma}^{-\varepsilon}} \widehat{f}(\xi)
$$

and is therefore supersymmetric. 
Proof Integration by parts gives

$$
\begin{aligned}
& -\varepsilon \kappa_{\sigma}^{\varepsilon} \frac{\partial}{\partial \eta_{\sigma}^{\varepsilon}} \widehat{f}(\xi)=\int \mathrm{d} \Phi \mathrm{e}^{-\mathrm{i} \xi^{+} \Phi^{-}-\mathrm{i} \Phi^{+} \xi^{-}} \psi_{\sigma}^{-\varepsilon} \frac{\partial}{\partial \phi_{\sigma}^{-\varepsilon}} f(\Phi), \\
& \eta_{\sigma}^{\varepsilon} \frac{\partial}{\partial \kappa_{\sigma}^{\varepsilon}} \widehat{f}(\xi)=\int \mathrm{d} \Phi \mathrm{e}^{-\mathrm{i} \xi^{+} \Phi^{-}-\mathrm{i} \Phi^{+} \xi^{-}} \varepsilon \phi_{\sigma}^{-\varepsilon} \frac{\partial}{\partial \psi_{\sigma}^{-\varepsilon}} f(\Phi),
\end{aligned}
$$

provided that the integrands are in $L^{1}(\mathcal{S}, \mathscr{G})$. In the first line this is the case by assumption. In the second line, by Lemma 2.13 we have that

$$
-\varepsilon \phi_{\sigma}^{-\varepsilon} \frac{\partial}{\partial \psi_{\sigma}^{-\varepsilon}} f(\Phi)=\psi_{\sigma}^{\varepsilon} \frac{\partial}{\partial \phi_{\sigma}^{\varepsilon}} f(\Phi)
$$

and thus the integrand is in $L^{1}(\mathcal{S}, \mathscr{G})$ as well. Taking the difference of the two equations at fixed $\varepsilon$ and $\sigma$, and using (4.14) gives identity (4.12). Parity follows by using that $\mathrm{d} \Phi$ is invariant under $\Phi \mapsto-\Phi$.

Proof of Proposition 4.8 We shall skip many details since the proof is analogous to the one of Proposition 3.9. The superfunction $F_{\beta}$ is well-defined thanks to (H1) and satisfies IDB for any $0 \leq \beta \leq \tau \gamma$ thanks to (H2-II) $\tau$. As a consequence, $\widehat{F_{\beta}}(\xi)$ decays in norm faster than any power of $\kappa$ so that we can make sense of the dual SUSY integral in (2.53) when $E \rightarrow z_{ \pm}, \epsilon \geq 0$. We set $\widehat{\mu}_{\mathrm{X}}^{ \pm}=: \mathrm{e}^{\widehat{V}_{\mathrm{X}}^{ \pm}}$, apply the BBF formula and, by exploiting the decay properties of $\widehat{F_{\beta}}$, obtain a polymer expansion like the one in the proof of Proposition 3.9. The stability condition $\operatorname{Re}\left(\hat{V}_{\mathrm{X}}^{ \pm}\right)_{\emptyset}=\operatorname{Re} \pm \mathrm{i} \gamma \sum_{x, y \in \mathrm{X}} \kappa_{x}^{+} C_{x, y}^{E \pm \mathrm{i} \epsilon} \kappa_{y}^{-} \leq 0$ is satisfied, since $C^{E \pm \mathrm{i} \epsilon}=$ $(H-E \pm \mathrm{i} \epsilon) /\left((H-E)^{2}+\epsilon^{2}\right)$ with $H$ Hermitian and $\epsilon \geq 0$. Accordingly, $\mathrm{d} p_{\mathrm{T}}(s)$ is supported on $s$ such that $\operatorname{Re} \pm \mathrm{i} \sum_{x, y \in \mathrm{Y}} s_{x, y} \hat{v}_{x, y}^{ \pm}((\kappa, 0)) \leq 0$ respectively. In the final formula for $G_{N}$ we have swapped integration with respect to $\mathrm{d} p_{\mathrm{T}}(s)$ with the superintegral with respect to d $\xi_{\mathrm{Y}}$ because of the decay properties of $\widehat{F}_{\beta}$.

Again, we notice that $\widehat{\mu}_{\mathrm{Y}}^{ \pm}(\cdot, s)$ and $\hat{v}_{x, y}^{ \pm}$are even and supersymmetric by inspection. We have already pointed out that $F_{\beta}$ is even, supersymmetric and invariant under $U(1)^{\times S}$ fermionic transformation, see discussion before Proposition 2.14 . Since by assumption it satisfies IDB, by Lemma 4.10 we have that $\widehat{F_{\beta}}$ is even, supersymmetric and satisfies identity (4.12), or equivalently:

$$
\frac{\eta_{\sigma}^{\varepsilon}}{\left(1+\left|\kappa_{\sigma}^{\varepsilon}\right|\right)} \frac{\partial}{\partial \kappa_{\sigma}^{\varepsilon}} \widehat{F_{\beta}}(\xi)=-\varepsilon \frac{\kappa_{\sigma}^{-\varepsilon}}{\left(1+\left|\kappa_{\sigma}^{\varepsilon}\right|\right)} \frac{\partial}{\partial \eta_{\sigma}^{-\varepsilon}} \widehat{F_{\beta}}(\xi) .
$$

Following the argument below (3.16), we finally have that the superfunction $\xi \mapsto$ $\widehat{\mu}^{ \pm}(\xi, s)\left(\prod_{\left\{x^{\prime}, y^{\prime}\right\} \in \mathrm{T}} \hat{v}_{x^{\prime}, y^{\prime}}(\xi)\right) \widehat{F_{\beta}^{Y}}(\xi)$ satisfies the hypotheses of Proposition 2.12. The rest of the proof is identical to the one of Proposition 3.9.

\subsection{Proof of Theorem 4.2}

Thanks to $(\mathrm{H} 1)$ and $(\mathrm{H} 2-\mathrm{II})_{\tau}$ the representation in Proposition 4.8 is valid. We will prove that if $\gamma \delta^{-1}$ is small enough then for some constant $C_{K, M, p, \theta}$ the following bound holds true:

$$
\sup _{\sigma, \sigma^{\prime}}\left|\left(G_{N}\left(x, y ; z_{ \pm} \pm \mathrm{i} \epsilon\right)\right)_{\sigma, \sigma^{\prime}}\right| \leq\left(C_{K, M, p, \theta} / 2\right)^{N+2-\delta_{x, y}} \delta^{-N} \mathrm{e}^{-\theta \sqrt{\delta}|x-y|} .
$$


Plugging this bound in (4.8) proves the convergence of the expansion and the claim. The proof of the bound (4.16) follows closely the strategy in the proof of Theorem 3.2, with some small differences which will be stressed.

The setting is as in the proof of Theorem 3.2, but we shall recall it for the sake of clarity. Let us consider a polymer $\mathrm{Y} \ni x, y$ and an oriented tree $\mathrm{T}_{\wp}$ on $\mathrm{Y}, \wp$ denoting the choice of orientations on the links of the tree. We denote by $\ell^{+}\left(\ell^{-}\right)$the starting (ending) vertex of the oriented link $\ell \in \mathrm{T}_{\wp}$. Links have to be oriented in order to select one of the two elements in $\hat{v}_{\ell}^{ \pm}(\xi)$, see in (4.10). Furthermore, we introduce the sequences $\underline{\sigma}=\left\{\sigma_{\ell}^{\varepsilon} \in \mathrm{S}\right\}_{\ell \in \mathrm{T}, \varepsilon= \pm}$ and $\underline{\sharp}=\left\{\sharp \sharp_{\ell} \in\{B, F\}\right\}_{\ell \in \mathrm{T}}$. We set $\xi_{B, x, \sigma}^{\varepsilon}=\kappa_{x, \sigma}^{\varepsilon}$ and $\xi_{F, x, \sigma}^{\varepsilon}=\eta_{x, \sigma}^{\varepsilon}$, and we shall henceforth write $|\mathrm{Y}|$ instead of $N+2-\delta_{x, y}=|\mathrm{Y}|$. Let us define:

$$
\widehat{\mathcal{F}}_{\mathrm{T}_{\wp}, \underline{\underline{y}}, \underline{\sigma}}^{\mathrm{Y}}(\xi):=\left(\prod_{\ell \in \mathrm{T}_{\wp}} \xi_{\sharp \ell, x_{\ell^{+}}, \sigma_{\ell}^{+}}^{+} \xi_{\sharp \ell, x_{\ell^{-}}, \sigma_{\ell}^{-}}^{-}\right) \frac{\partial}{\partial \eta_{x}^{+}} \frac{\partial}{\partial \eta_{y}^{-}} \widehat{F}_{\beta}^{\mathrm{Y}}(\xi) .
$$

We can rewrite (4.9) as

$$
\begin{aligned}
G_{N}\left(x, y ; z_{ \pm} \pm \mathrm{i} \epsilon\right)= & \frac{1}{N !} \sum_{\substack{\mathrm{T} \text { on } \\
\{1, \ldots,|\mathrm{Y}|\}}} \sum_{\wp, \underline{\sharp}, \underline{\sigma} \underline{\sigma}_{x_{1}, \ldots, x_{N} \in \Lambda^{\prime}}} \sum_{\text {distinct }} \int \mathrm{d} p_{\mathrm{T}}(s) \cdot \\
& \cdot \int \mathrm{d} \widehat{\mu}_{\mathrm{Y}}^{ \pm}(\xi, s) \widehat{\mathcal{F}}_{\mathrm{T}_{\wp}, \underline{\sharp}, \underline{\sigma}}^{\mathrm{Y}}(\xi)\left(\prod_{\ell \in \mathrm{T}_{\wp}}\left(C_{x_{\ell^{+}}, x_{\ell^{-}}}^{E \pm \mathrm{i}^{-}}\right)_{\sigma_{\ell}^{+}, \sigma_{\ell}^{-}}\right),
\end{aligned}
$$

which can be bounded as follows:

$$
\begin{aligned}
& \sup _{\sigma, \sigma^{\prime}}\left|\left(G_{N}\left(x, y ; z_{ \pm} \pm \mathrm{i} \epsilon\right)\right)_{\sigma, \sigma^{\prime}}\right|
\end{aligned}
$$

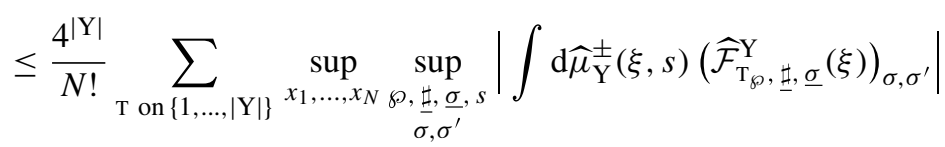

$$
\begin{aligned}
& \sup _{\wp} \sum_{\underline{\sigma}} \sum_{\substack{x_{1}, \ldots, x_{N} \in \Lambda^{\prime} \\
\text { distinct }}} \prod_{\ell \in \mathrm{T}_{\wp}}\left|\left(C_{x_{\ell^{+}} \pm x_{\ell^{-}}}^{E \pm{ }^{-}}\right)_{\sigma_{\ell}^{+}, \sigma_{\ell}^{-}}\right|,
\end{aligned}
$$

where the $\sup _{s}$ is taken over $s$ in the support of $\mathrm{d} p_{\mathrm{T}}$. We shall first obtain a useful bound for the superintegral. The analysis differs slightly from the one of the proof of Theorem 3.2 because we need to prove suitable decay bounds for $\left\|\left(\widehat{\mathcal{F}}_{\mathrm{T}_{\wp}, \underline{\sharp}, \underline{\sigma}}^{\mathrm{Y}}(\xi)\right)_{\sigma, \sigma^{\prime}}\right\|$. We again see by inspection that $\widehat{\mathcal{F}}_{\mathrm{T}_{\wp}, \underline{\sharp}, \underline{\sigma}}^{\mathrm{Y}}(\xi)$ is a local function:

$$
\widehat{\mathcal{F}}_{\mathrm{T}_{\wp}, \underline{\underline{\sigma}}, \underline{\sigma}}^{\mathrm{Y}}(\xi)= \pm \prod_{i=1}^{|\mathrm{Y}|} \widehat{\mathcal{F}}_{x, y} \frac{d_{i}}{\left(\xi_{x_{i}}\right)}
$$

having set:

$$
\widehat{\mathcal{F}}_{x, y}^{d}\left(\xi_{x^{\prime}}\right):=\left(\prod_{\varepsilon, \sigma, \sharp}\left(\xi_{\sharp, x^{\prime}, \sigma}^{\varepsilon}\right)^{d_{\sharp, \sigma}^{\varepsilon}}\right)\left(\frac{\partial}{\partial \eta_{x}^{+}}\right)^{\delta_{x^{\prime}, x}}\left(\frac{\partial}{\partial \eta_{y}^{-}}\right)^{\delta_{x^{\prime}, y}} \widehat{F_{\beta}}\left(\xi_{x^{\prime}}\right),
$$




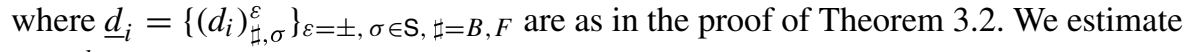
$\left.\|\left(\widehat{\mathcal{F}}_{\bar{x}, y} \frac{d_{i}}{\xi_{x_{i}}}\right)\right)_{\sigma, \sigma^{\prime}} \|$ by means of the IDB on $F_{\beta}$. In fact, for any $m \in \mathbb{N}$, by Lemma 2.10:

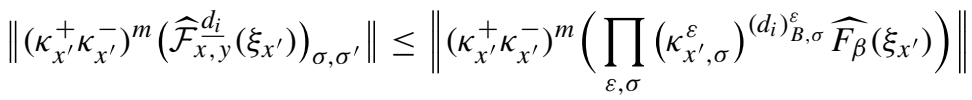

$$
\begin{aligned}
& \leq \int \mathrm{d} \phi_{x^{\prime}}\left\|\left[\left(\frac{\partial}{\partial \phi_{x^{\prime}}^{+}}\right)\left(\frac{\partial}{\partial \phi_{x^{\prime}}^{-}}\right)\right]^{m}\left(\prod_{\varepsilon, \sigma}\left(\frac{\partial}{\partial \phi_{x^{\prime}}^{\varepsilon}}\right)^{\left(d_{i}\right)_{B, \sigma}^{\varepsilon}}\right) F_{\beta}\left(\Phi_{x^{\prime}}\right)\right\| ;
\end{aligned}
$$

thus, by the IDB on $F_{\beta}$ we obtain:

$$
\left\|\widehat{\mathcal{F}}_{x, y}^{\frac{d_{i}}{x, y}}\left(\xi_{x_{i}}\right)\right\| \leq 2 K|\mathrm{~S}|^{m} \frac{\left(\left(d_{i, B}+2 m\right) !\right)^{p} M^{d_{i, B}}}{1+M^{-2 m}\left(\kappa_{x_{i}}^{+} \kappa_{x_{i}}^{-}\right)^{m}}, \quad \forall m \in \mathbb{N} .
$$

Finally, since $M \geq 1$

$$
\sup _{\wp, \sharp, \underline{\sigma}}\left\|\widehat{\mathcal{F}}_{\mathrm{T}_{\wp}, \underline{\sharp}, \underline{\sigma}}^{\mathrm{Y}}(\xi)\right\| \leq \prod_{i=1}^{|\mathrm{Y}|} 2 K|\mathrm{~S}|^{m} \frac{\left(\left(d_{i}+2 m\right) !\right)^{p} M^{d_{i}}}{1+M^{-2 m}\left(\kappa_{x_{i}}^{+} \kappa_{x_{i}}^{-}\right)^{m}}, \quad \forall m \in \mathbb{N} .
$$

On the other hand, the non-local part in the superintegral is bounded as:

$$
\sup _{s}\left\|\widehat{\mu}_{\mathrm{Y}}^{ \pm}(\xi, s)\right\| \leq \mathrm{e}^{\gamma\left\|C^{E \pm \mathrm{i} \epsilon}\right\|_{\infty, 1}|\mathrm{Y}|}
$$

where $\left\|C^{E \pm \mathrm{i} \epsilon}\right\|_{\infty, 1} \leq \mathcal{C} \delta^{-1}$ is bounded thanks to (4.2), where for simplicity we have dropped the dependence of $\mathcal{C}$ on $\mathrm{D}$, the dimension of the lattice. All in all, if we apply the bound in (4.24) with $m=|\mathrm{S}|+1$ and use $\left|\int \mathrm{d} \xi_{\mathrm{Y}} f(\xi)\right| \leq\|f\|_{L^{1}\left(\mathcal{S}^{\mathrm{Y}}, \mathscr{G}^{\mathrm{Y}}\right)}$, we finally obtain:

$$
\begin{aligned}
& \sup _{\substack{\wp, \underline{\sharp}, \underline{\sigma}, s \\
\sigma, \sigma^{\prime}}}\left|\int \mathrm{d} \widehat{\mu}_{\mathrm{Y}}^{ \pm}(\xi, s)\left(\mathcal{F}_{\mathrm{T}_{\wp}, \underline{\sharp}, \underline{\sigma}}^{\mathrm{Y}}(\xi)\right)_{\sigma, \sigma^{\prime}}\right| \\
& \quad \leq\left(K^{\prime}|\mathrm{S}|^{|\mathrm{S}|+1} M^{2|\mathrm{~S}|} \mathrm{e}^{\gamma\left\|C^{E \pm \mathrm{i} \epsilon}\right\|_{\infty, 1}}\right)^{|\mathrm{Y}|} \prod_{i=1}^{|\mathrm{Y}|}\left(\left(d_{i}+2|\mathrm{~S}|+2\right) !\right)^{p} M^{d_{i}} .
\end{aligned}
$$

for some $K^{\prime}>K$. To estimate the second line in (4.19), the strategy is the same as in the proof of Theorem 3.2. We exploit the exponential decay of the covariance (4.2). If we define $\left(C_{\theta}^{E \pm \mathrm{i} \epsilon}\right)_{x, y}:=\mathrm{e}^{(1+\theta) \sqrt{\delta}|x-y| / 2} C_{x, y}^{E \pm \mathrm{i} \epsilon}$, it follows that

$$
\begin{aligned}
& \left\|C_{\theta}^{E \pm \mathrm{i} \epsilon}\right\|_{\infty, 1}=\sup _{x \in \mathbb{Z}^{\mathrm{D}}} \sum_{x^{\prime} \in \mathbb{Z}^{\mathrm{D}}} \mathrm{e}^{(1+\theta) \sqrt{\delta}\left|x^{\prime}\right| / 2} \sum_{\sigma, \sigma^{\prime} \in \mathrm{S}}\left|\left(C_{x, x^{\prime}}^{E \pm \mathrm{i} \epsilon}\right)_{\sigma, \sigma^{\prime}}\right| \leq \mathcal{C}_{\theta} \delta^{-1} \\
& \left\|C_{\theta}^{E \pm \mathrm{i} \epsilon}\right\|_{\infty, \infty}=\sup _{x, x^{\prime} \in \mathbb{Z}^{\mathrm{D}}} \mathrm{e}^{(1+\theta) \sqrt{\delta}\left|x^{\prime}\right| / 2} \sum_{\sigma, \sigma^{\prime} \in \mathrm{S}}\left|\left(C_{x, x^{\prime}}^{E \pm \mathrm{i} \epsilon}\right)_{\sigma, \sigma^{\prime}}\right| \leq \mathcal{C}
\end{aligned}
$$


for some new $\mathcal{C}_{\theta}>\mathcal{C}$. Standard tree-stripping estimates (see Fig. 1) based on the exponential decay of $C^{E \pm \mathrm{i} \epsilon}$ give [16]:

$$
\begin{aligned}
& \sum_{\underline{\sigma}} \sum_{\substack{x_{1}, \ldots, x_{N} \in \Lambda^{\prime} \\
\text { distinct }}} \prod_{\ell \in \mathrm{T}_{\wp}}\left|\left(C_{x_{\ell^{+}}+x_{\ell^{-}}}^{E \pm \mathrm{i} \epsilon}\right)_{\sigma_{\ell}^{+}, \sigma_{\ell}^{-}}\right| \\
& \leq \mathrm{e}^{-\theta \sqrt{\delta}|x-y|} \sum_{\underline{\sigma}} \sum_{\substack{x_{1}, \ldots, x_{N} \in \Lambda^{\prime} \\
\text { distinct }}} \prod_{\ell \in \mathrm{T}_{\wp}}\left|\left(\left(C_{2 \theta-1}^{E \pm \mathrm{i} \epsilon}\right)_{x_{\ell^{+}}, x_{\ell^{-}}}\right)_{\sigma_{\ell}^{+}, \sigma_{\ell}^{-}}\right| \\
& \leq \mathrm{e}^{-\theta \sqrt{\delta}|x-y|}\left\|C_{\theta}^{E \pm \mathrm{i} \epsilon}\right\|_{\infty, 1}^{N}\left\|C_{\theta}^{E \pm \mathrm{i} \epsilon}\right\|_{\infty, \infty}^{1-\delta_{x, y}} \prod_{i=1}^{|\mathrm{Y}|}\left(C_{q, \theta}\right)^{d_{i}-1}\left(d_{i} !\right)^{-q} .
\end{aligned}
$$

The details on the tree-stripping procedure are explained in (3.33), text around it and in Fig. 1. Finally, we plug the bounds (4.25) (recall that $\left.\delta^{-1} \gamma \leq 1\right)$, (4.26) and (4.28) with $q=p+1$ into (4.19), and we use Cayley's theorem on the number of trees with fixed coordination numbers $\left\{d_{i}\right\}_{i}$, see [16], to obtain:

$$
\begin{aligned}
\left|\left(G_{N}\left(x, y ; z_{ \pm} \pm \mathrm{i} \epsilon\right)\right)_{\sigma, \sigma^{\prime}}\right| & \leq \mathrm{e}^{-\theta \sqrt{\delta}|x-y|}\left(K^{\prime}|\mathrm{S}|{ }^{|\mathrm{S}|+1} M^{2|\mathrm{~S}|} \mathrm{e}^{\mathcal{C}}\right)^{|\mathrm{Y}|} \mathcal{C}\left(\mathcal{C}_{\theta} \delta^{-1}\right)^{N} \\
& \sum_{\left\{d_{i}\right\}_{i}, d_{i} \geq 1} \prod_{i=1} \frac{|\mathrm{Y}|}{\sum_{i} d_{i}=2|\mathrm{Y}|-2} \\
\leq & \left(C_{K, M, p, \theta} / 2\right)^{|\mathrm{Y}|} \delta^{-N} \mathrm{e}^{-\theta \sqrt{\delta}|x-y|}
\end{aligned}
$$

where $C_{K, M, p, \theta}:=2 K^{\prime}|\mathrm{S}|^{|\mathrm{S}|+1} M^{2|\mathrm{~S}|+1} \mathrm{e}^{\mathcal{C}} \mathcal{C}_{\theta} \sum_{n=0}^{\infty} \frac{((n+2|\mathrm{~S}|+2) !)^{p}\left(M C_{p+1, \theta}\right)^{n}}{(n !)^{p+2}}<\infty$.

\subsection{Proof of Theorem 4.5}

To prove Theorem 4.5, we need to further expand the super Gibbs' weight. We will need the following lemma.

Lemma 4.11 (Super Lagrange Remainder) Let $f: \mathcal{S}^{\mathrm{X}} \rightarrow \mathscr{G}^{\mathrm{X}}$. If $\|f(\Phi)\|<\infty$, then the following Lagrange estimate holds

$$
\left\|\mathrm{e}^{f(\Phi)}-\sum_{j=0}^{k} \frac{(f(\Phi))^{j}}{j !}\right\| \leq \frac{1}{(k+1) !} \sup _{t \in(0,1)}\left\|\mathrm{e}^{t f(\Phi)}(f(\Phi))^{k+1}\right\| .
$$

Proof Since $\|f(\Phi)\|$ is finite, the function $t \mapsto \mathrm{e}^{t f(\Phi)}=\sum_{n \geq 0}(t f(\Phi))^{n} / n ! \in \mathscr{G}^{\mathrm{X}}$ is analytic. As usual, define the integral Lagrange remainder

$$
R_{k}(\Phi):=\int_{0}^{1} \mathrm{~d} t \frac{(1-t)^{k}}{k !}\left(\frac{\mathrm{d}}{\mathrm{d} t}\right)^{k} \mathrm{e}^{t f(\Phi)}=\mathrm{e}^{f(\Phi)}-\sum_{j=0}^{k} \frac{(f(\Phi))^{j}}{j !},
$$

which we estimate in Grassmann norm, hence the claim. 
For the sake of brevity, we shall write $G_{N}(E+\mathrm{i} \epsilon):=G_{N}(0,0 ; E+\mathrm{i} \epsilon)$. By Proposition 4.8 we have that for $E \in \mathbb{R}$

$$
\rho_{\epsilon, \Lambda}(E)=-\frac{\gamma^{-1}}{\pi|\mathrm{S}|} \sum_{N=0}^{|\Lambda|} \operatorname{Re}\left((\mathrm{i} \gamma)^{N} \operatorname{Tr}_{S} G_{N}(E+\mathrm{i} \epsilon)\right) .
$$

Furthermore, by the proof of Theorem 4.2 the following bound holds for $C_{K, M, p}:=$ $C_{K, M, p, \theta=0}$ :

$$
\sup _{\sigma}\left|\left(G_{N}(E+\mathrm{i} \epsilon)\right)_{\sigma, \sigma}\right| \leq\left(C_{K, M, p} / 2\right)^{N+1} \delta^{-N},
$$

provided that $\delta \geq \gamma C_{M, K, p}$. Let us now fix $\bar{N} \in \mathbb{N}$ sufficiently large to be optimized later. To prove the claim, it suffices to improve the bound (4.33) for $N<\bar{N}$ as follows:

$$
\sup _{\sigma}\left|\operatorname{Re}\left((\mathrm{i} \gamma)^{N}\left(G_{N}(E+\mathrm{i} \epsilon)\right)_{\sigma, \sigma}\right)\right| \leq(\bar{N} !)^{2 p}\left(\gamma \delta^{-1} \widetilde{C}_{M, p}\right)^{\bar{N}}\left(\widetilde{C}_{K, M, p}\right)^{N+1}
$$

for some constants $\widetilde{C}_{M, p}$ and $\widetilde{C}_{K, M, p}$. Indeed, if the bounds (4.33) and (4.34) hold true, then for any $\bar{N}$ and for some other constant $C_{K, M, p}^{\prime}$

$$
\left|\rho_{\epsilon, \Lambda}(E)\right| \leq \gamma^{-1} C_{K, M, p}^{\prime}(\bar{N} !)^{2 p}\left(C_{K, M, p}^{\prime} \gamma \delta^{-1}\right)^{\bar{N}}
$$

Therefore, by minimizing over $\bar{N} \in \mathbb{N}$ we obtain the statement for a suitable constant $\bar{C}_{K, M, p}$ :

$$
\left|\rho_{\Lambda}(E)\right| \leq \gamma^{-1} C_{K, M, p}^{\prime} \mathrm{e}^{-\bar{C}_{K, M, p}\left(\gamma^{-1} \delta\right)^{1 / 2 p}} .
$$

Let us now prove (4.34). To begin, we Taylor expand the super Gibbs' weight $\widehat{\mu}_{\mathrm{Y}}^{+}(\xi, s)$ :

$$
\widehat{\mu}_{\mathrm{Y}}^{+}(\xi, s)=\sum_{j=0}^{\bar{N}-N-1} \frac{(\mathrm{i} \gamma)^{j}}{j !}\left(\frac{1}{2} \sum_{x, y \in \mathrm{Y}} s_{x, y} \hat{v}_{x, y}^{+}(\xi)\right)^{j}+\operatorname{Rem}_{\bar{N}-N} \widehat{\mu}_{\mathrm{Y}}^{+}(\xi, s)
$$

and accordingly introduce the splitting,

$$
G_{N}(E+\mathrm{i} \epsilon)=G_{\bar{N}, N}(E+\mathrm{i} \epsilon)+R_{\bar{N}, N}(E+\mathrm{i} \epsilon)
$$

where

$$
\begin{aligned}
& G_{\bar{N}, N}(E+\mathrm{i} \epsilon):= \\
& =\frac{1}{N !} \sum_{l=0}^{\bar{N}-N-1} \frac{(\mathrm{i} \gamma)^{l}}{l !} \sum_{\substack{\mathrm{T} \text { on } \\
\{1, \ldots, N+1\}}} \sum_{\substack{x_{1}, \ldots, x_{N} \in \Lambda^{\prime} \\
\text { distinct }}} \int \mathrm{d} p_{\mathrm{T}}(s) \int \mathrm{d} \xi^{\mathrm{Y}} . \\
& \cdot\left(\sum_{x, y \in \mathrm{Y}} \frac{1}{2} s_{x, y} \hat{v}_{x, y}^{+}(\xi)\right)^{l} \prod_{\{i, j\} \in \mathrm{T}} \hat{v}_{x_{i}, x_{j}}^{+}(\xi) \frac{\partial}{\partial \eta_{x}^{+}} \frac{\partial}{\partial \eta_{y}^{-}} \widehat{F}_{0}^{\mathrm{Y}}(\xi) \\
& =\frac{1}{N !} \sum_{l=0}^{\bar{N}-N-1} \frac{(\mathrm{i} \gamma)^{l}}{l !} \sum_{\substack{\mathrm{T} \text { on } \\
\{1, \ldots, N+1\}}} \sum_{\substack{x_{1}, \ldots, x_{N} \in \Lambda^{\prime} \\
\text { distinct }}} \int \mathrm{d} p_{\mathrm{T}}(s) . \\
& \left.\cdot\left(-\frac{1}{2} \sum_{x, y \in \mathrm{Y}} s_{x, y} \hat{v}_{x, y}^{+}(\partial / \partial \Phi)\right)^{l} \prod_{\{i, j\} \in \mathrm{T}} \hat{v}_{x_{i}, x_{j}}^{+}(\partial / \partial \Phi) \psi_{0}^{-} \psi_{0}^{+} F_{0}^{\mathrm{Y}}(\Phi)\right|_{\Phi=0}
\end{aligned}
$$

with $\hat{v}_{x, y}^{+}(\partial / \partial \Phi):=\partial / \partial \Phi_{x}^{+} C_{x, y}^{E+\mathrm{i} \epsilon} \partial / \partial \Phi_{y}^{-}+\partial / \partial \Phi_{y}^{+} C_{y, x}^{E+\mathrm{i} \epsilon} \partial / \partial \Phi_{x}^{-}$. Since $v$ is even by assumption, $\hat{v}^{(2 n+1)}(0)=\mathbb{E} \omega^{2 n+1}=0$ and thus $\psi_{0}^{-} \psi_{0}^{+} F_{0}^{\mathrm{Y}}(\Phi)$ has non-vanishing 
derivatives in $\Phi=0$ only of order $2(2 n+1), n \in \mathbb{N}$. As a consequence, only the terms such that $l+N$ is odd survive in the sum above. Finally, $C_{x, y}^{E+\mathrm{i} 0^{+}} \in \mathbb{R}$ for any $x, y$, and $\hat{v}^{(2 n)}(0) \in \mathbb{R}$ imply that $G_{\bar{N}, N}\left(E+\mathrm{i} 0^{+}\right)$does not contribute to $\rho_{\Lambda}(E)$, that is

$$
\operatorname{Re}\left((\mathrm{i} \gamma)^{N} G_{\bar{N}, N}\left(E+\mathrm{i} 0^{+}\right)\right)=0 .
$$

We are left with bounding $\left(R_{\bar{N}, N}(E+\mathrm{i} \epsilon)\right)_{\sigma, \sigma}$ :

$$
\begin{aligned}
& R_{\bar{N}, N}(E+\mathrm{i} \epsilon)=\frac{1}{N !} \sum_{\substack{\mathrm{T} \text { on } \\
\{1, \ldots, N+1\}}} \sum_{\wp, \underline{\sharp}, \underline{\sigma} \sum_{x_{1}, \ldots, x_{N} \in \Lambda^{\prime}}} \sum_{\text {distinct }} \int \mathrm{d} p_{\mathrm{T}}(s) \cdot \\
& \cdot \int \mathrm{d} \xi_{\mathrm{Y}}\left(\operatorname{Rem}_{\bar{N}-N-1} \widehat{\mu}_{\mathrm{Y}}^{+}(\xi, s)\right) \mathcal{F}_{\mathrm{T}_{\wp}, \underline{\sharp}, \underline{\sigma}}^{\mathrm{Y}}(\xi)\left(\prod_{\ell \in \mathrm{T}_{\wp}}\left(C_{x_{\ell^{+}}, x_{\ell^{-}}}^{E \pm \mathrm{i} \epsilon}\right)_{\sigma_{\ell}^{+}, \sigma_{\ell}^{-}}\right),
\end{aligned}
$$

where the notation is again borrowed from the previous proof, see (4.17) and the text above. We introduce $\tilde{x}=\left(\tilde{x}^{+}, \tilde{x}^{-}\right) \in\left(\mathrm{Y}^{\bar{N}-N}\right)^{2}, \tilde{\sharp} \in\{B, F\}^{\bar{N}-N}$ and $\tilde{\sigma}=\left(\tilde{\sigma}^{+}, \tilde{\sigma}^{-}\right) \in\left(\mathrm{S}^{\bar{N}-N}\right)^{2}$. For any $\tilde{x}, \tilde{\sharp}$ and $\tilde{\sigma}$, we define a sequence $\underline{\tilde{d}}_{i}:=$ $\left\{\left(\tilde{d}_{i}\right)_{\sharp, \sigma}^{\varepsilon}\right\}_{\varepsilon= \pm, \sharp=B, F}$ at any vertex $i \in\{1, \ldots, N+1\}$ of the tree, where $\left(\tilde{d}_{i}\right)_{\sharp, \sigma}^{\varepsilon}:=$ $\sum_{j=1}^{\bar{N}-N} \delta_{x_{i}, \tilde{x}_{j}^{\varepsilon}} \delta_{\sharp, \tilde{\sharp}_{j}} \delta_{\sigma, \tilde{\sigma}_{j}^{\varepsilon}}$. Furthermore, we define the following local function:

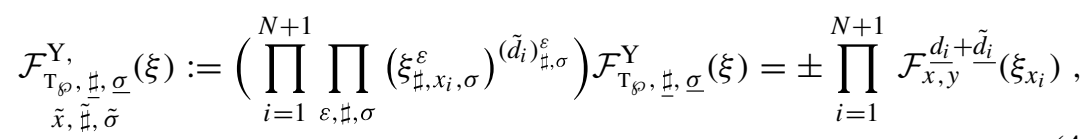

where again the sign is unimportant for our purposes and where $\widehat{\mathcal{F}}_{x, y}^{d}\left(\xi_{x^{\prime}}\right)$ was introduced in (4.21). Finally, by Lemma 4.11, we estimate $\operatorname{Rem}_{\bar{N}-N}$ in Grassmann norm as a super Lagrange remainder:

$$
\begin{aligned}
& \left|\left(R_{\bar{N}, N}(E+\mathrm{i} \epsilon)\right)_{\sigma, \sigma}\right| \leq \frac{4^{N+1}}{N !} \frac{(2 \gamma)^{\bar{N}-N}}{(\bar{N}-N) !}
\end{aligned}
$$

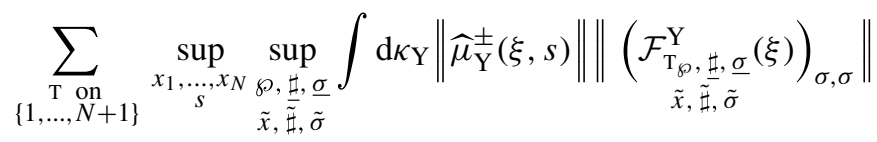

$$
\begin{aligned}
& \times\left(\sup _{\wp} \sum_{\underline{\sigma}} \sum_{\substack{x_{1}, \ldots, x_{N} \in \Lambda^{\prime} \\
\text { distinct }}} \prod_{\ell \in \mathrm{T}_{\wp}}\left|\left(C_{x_{\ell^{+}}, x_{\ell^{-}}}^{E+\mathrm{i} \epsilon}\right)_{\sigma_{\ell}^{+}, \sigma_{\ell}^{-}}\right|\right) \\
& \times\left(\sum_{\tilde{x}, \tilde{\sigma}} \prod_{j=1}^{\bar{N}-N}\left|\left(C_{\tilde{x}_{j}^{+}, \tilde{x}_{j}^{-}}^{E+\mathrm{i} \epsilon}\right)_{\tilde{\sigma}_{j}^{+}, \tilde{\sigma}_{j}^{-}}\right|\right) .
\end{aligned}
$$

In order to bound the integral, we follow the strategy used in the proof of Theorem 4.2. Using (4.42) we obtain that

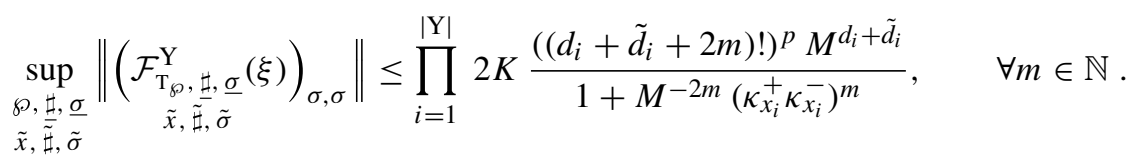


Thus:

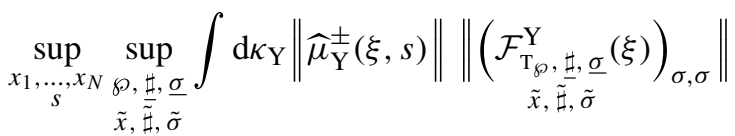

$$
\begin{aligned}
& \leq\left(K_{M}^{\prime}\right)^{|\mathrm{Y}|}((2 \bar{N}-2 N) !)^{p} \prod_{i=1}^{|\mathrm{Y}|}\left(\left(d_{i}+2|\mathrm{~S}|+2\right) !\right)^{p}\left(2^{p} M\right)^{d_{i}+\tilde{d}_{i}}
\end{aligned}
$$

for some constant $K_{M}^{\prime}$ depending on $|\mathrm{S}|$ as well. Finally, by noticing that

$$
\sum_{\tilde{x}, \tilde{\sigma}} \prod_{j=1}^{\bar{N}-N}\left|\left(C_{\tilde{x}_{j}^{+}, \tilde{x}_{j}^{-}}^{E+\mathrm{i} \epsilon}\right)_{\tilde{\sigma}_{j}^{+}, \tilde{\sigma}_{j}^{-}}\right| \leq\left(|\mathrm{Y}||\mathrm{S}|\left\|C^{E+\mathrm{i} \epsilon}\right\|_{\infty, 1}\right)^{\bar{N}-N}
$$

we obtain the following bound for some constant $\widetilde{C}_{M, p}$,

$$
\left|\left(R_{\bar{N}, N}(E+\mathrm{i} \epsilon)\right)_{\sigma, \sigma}\right| \leq(\bar{N} !)^{2 p}\left(\delta^{-1} \widetilde{C}_{M, p}\right)^{\bar{N}} \gamma^{\bar{N}-N}\left(C_{K, 2^{p} M, p, \theta=0}\right)^{N+1},
$$

which implies the desired estimate (4.34).

\section{Appendix A: Disorder Distribution}

We discuss two examples that connect the properties of the density $v$ with the IMB contained in hypothesis $(\mathrm{H} 2-\mathrm{I})_{\tau}$, see Definition 3.1 and below:

Example I.1 We can state the following lemma.

Lemma A.1 Let $v \in \mathscr{S}(\mathbb{R})$ be analytic in the strip $\mid$ Im $t \mid<W$. Then, if $\tau<W, F_{\tau \gamma}$ satisfies IMB with $M=(W / 2-\tau / 2)^{-1 / 2}$ and $p=1 / 2$.

Proof The assumptions on $v$ imply that $\left\|\hat{v}\left(\Phi^{+} \Phi^{-}\right)\right\| \leq C \mathrm{e}^{-W \phi^{+} \phi^{-}}$, for some universal constant $C$. Using that $\left|\left(\phi_{\sigma}^{+}\right)^{n_{\sigma}^{+}}\left(\phi_{\sigma}^{-}\right)^{n_{\sigma}^{-}}\right| \leq\left(n_{\sigma} / 2\right) ! M^{-n_{\sigma} / 2} \mathrm{e}^{M \phi_{\sigma}^{+} \phi_{\sigma}^{-}}$, where $n_{\sigma}=n_{\sigma}^{+}+n_{\sigma}^{-}$and $M=W / 2-\tau / 2$ the claim follows. Here $K$ grows with $M^{-1}$.

Notice that Example I.1 include Gaussian distributions.

Example I.2 The decay of the Fourier transform of test functions can be quantitatively characterised, see, e.g., [37]. For instance, if $v(t)=\mathrm{e}^{-\frac{1}{1-t^{2}}} \mathbf{1}_{|t| \leq 1}$, so that $F_{0}$ satisfies IMB with $M=2$ and $p=1$.

We then discuss two examples that connect the properties of the density $v$ with the IDB contained in hypothesis $(\mathrm{H} 2-\mathrm{II})_{\tau}$, see Definition 4.1 and below. Both examples include Gaussian distributions. 
Example II.1 Introduce the following seminorm on functions of a supervector:

$$
\|f\|_{1, W}:=\sup _{\zeta \in \mathbb{R}_{W}^{2}} \int_{\mathbb{R}^{2 S}} \mathrm{~d} \phi\|f(\Phi+(\zeta, 0))\|,
$$

where $\mathbb{R}_{W}^{2 \mathrm{~S}}=\left\{\phi \in \mathbb{C}^{2 \mathrm{~S}}|| \operatorname{Im} \phi_{i, \sigma} \mid \leq W, i=1,2, \sigma \in \mathrm{S}\right\}$. We state the following lemma.

Lemma A.2 Assume that for any $\alpha \in \mathbb{R}$, $\mathrm{e}^{\alpha|t|} \nu(t)$ is bounded and that $\left\|F_{\tau \gamma}\right\|_{1, W}$ is finite for some $0<W \leq 1$. Then $F_{\tau \gamma}$ satisfies $I D B$ with $K=\left\|F_{\tau \gamma}\right\|_{1, W}, M=W^{-1}$ and $p=1$.

Proof We notice that $\hat{v}$ is entire and so is $F_{\tau \gamma}$ in $\phi$. We thus apply the multi-variable Cauchy integral formula in $\mathbb{R}_{W}^{2 S}$. Accordingly,

$$
\left[\prod_{i, \sigma}\left(\frac{\partial}{\partial \phi_{i, \sigma}}\right)^{n_{i, \sigma}}\right] F_{\tau \gamma}(\Phi)=\left(\prod_{i, \sigma} \frac{n_{i, \sigma} !}{2 \pi \mathrm{i}}\right) \oint_{(\partial D(0, W))^{2 \mathrm{~S}}} \mathrm{~d} w \frac{F_{\tau \gamma}(\Phi+(w, 0))}{\prod_{i, \sigma} w_{i, \sigma}^{n_{i, \sigma}+1}},
$$

where $D(0, W)=\{w \in \mathbb{C}|| w \mid \leq W\}$ and $\mathrm{d} w=\times_{i, \sigma} \mathrm{d} w_{i, \sigma}$; therefore:

$$
\int \mathrm{d} \phi\left\|\left[\prod_{i, \sigma}\left(\frac{\partial}{\partial \phi_{i, \sigma}}\right)^{n_{i, \sigma}}\right] F_{\tau \gamma}(\Phi)\right\| \leq n ! W^{-n}\left\|F_{\tau \gamma}\right\|_{1, W},
$$

where $n=\sum_{i, \sigma} n_{i, \sigma}$. The claim follows because $\partial / \partial \phi_{\sigma}^{\varepsilon}=1 / 2\left(\partial / \partial \phi_{1, \sigma}\right.$ $\left.-\mathrm{i} \varepsilon \partial / \partial \phi_{2, \sigma}\right)$.

Example II.2 Introduce the following seminorm on functions of a supervector:

$$
\|f\|_{1, W}:=\sup _{\zeta \in(\partial D(0, W))^{2 S}} \int_{\mathbb{R}^{2 S}} \mathrm{~d} \phi \mathrm{e}^{\sum_{\sigma \in \mathrm{S}}\left(\phi_{\sigma}^{+} \phi_{\sigma}^{-}\right)^{1 / 2}}\|f(\Phi+(\zeta, 0))\|,
$$

where $D(0, W) \subset \mathbb{C}$ was defined above. We state the following lemma.

Lemma A.3 Assume $\mathrm{e}^{W|t|} v(t)$ is bounded and $\left\|F_{\tau \gamma}\right\|_{1, W}$ is finite for some $0<W \leq$ 1. Then $F_{\tau \gamma}$ satisfies IDB with $K=|\mathrm{S}| ! 2^{|s|}\left\|\mid F_{\tau \gamma}\right\|_{1, W}, M=4\left(W^{-1}+\tau\right), p=2$.

Proof We notice that $\hat{v}(t)$ is holomorphic on the strip $|\operatorname{Im} t| \leq W$ which we shall use to estimate its derivative. We think of $F_{\tau \gamma}(\Phi)$ as the composite function $f\left(\Phi^{+} \Phi^{-}\right)$ and compute its derivative accordingly. For simplicity, we carry out the computation in the case of $|\mathrm{S}|=1$ and we take, e.g., $n_{\sigma}^{+} \geq n_{\sigma}^{-}$and $n_{\sigma}=n_{\sigma}^{+}+n_{\sigma}^{-}$. We accordingly bound the norm of the derivative as follows:

$$
\left\|\left(\prod_{\varepsilon= \pm}\left(\frac{\partial}{\partial \phi_{\sigma}^{\varepsilon}}\right)^{n_{\sigma}^{\varepsilon}}\right) F_{z}(\Phi)\right\| \leq 2^{n_{\sigma}} \sum_{i=0}^{n_{\sigma}^{-}} i !|\phi|^{n_{\sigma}-2 i}\left\|f^{\left(n_{\sigma}-i\right)}\left(\Phi^{+} \Phi^{-}\right)\right\| .
$$

We apply the Cauchy integral representation to estimate the derivatives of $f(\cdot)$ and we use the bound $|\phi|^{n_{\sigma}-2 i} \leq\left(n_{\sigma}-2 i\right) ! \mathrm{e}^{\left(\phi_{\sigma}^{+} \phi_{\sigma}^{-}\right)^{1 / 2}}$. Integrating in $\mathrm{d} \phi_{\sigma}$ and taking the superior over the contour variable gives the claim. 
To conclude this appendix, we will show that requiring either IMB or IDB over $[0, \tau]$, respectively in $(\mathrm{H} 2-\mathrm{I})_{\tau}$ and $(\mathrm{H} 2-\mathrm{II})_{\tau}$, with $K, M$ and $p$ depending on $\tau$, is very natural. In fact, if $F_{\tau^{\prime} \gamma}(\Phi)=\mathrm{e}^{\tau \Phi^{+} \Phi^{-}} \hat{v}\left(\Phi^{+} \Phi^{-}\right)$satisfies either IMB or IDB for $\tau^{\prime}=\tau$, then so does it for any $\tau^{\prime} \in[0, \tau]$. In the case of IMB, this is a simple consequence of the following inequality

$$
\begin{aligned}
& \left\|\left(\prod_{\varepsilon, \sigma}\left(\phi_{\sigma}^{\varepsilon}\right)^{n_{\sigma}^{\varepsilon}}\right) \mathrm{e}^{\tau^{\prime} \Phi^{+} \Phi^{-}} \hat{v}\left(\Phi^{+} \Phi^{-}\right)\right\| \\
= & \left\|\mathrm{e}^{-\left(\tau-\tau^{\prime}\right) \Phi^{+} \Phi^{-}}\left(\prod_{\varepsilon, \sigma}\left(\phi_{\sigma}^{\varepsilon}\right)^{n_{\sigma}^{\varepsilon}}\right) F_{\tau \gamma}(\Phi)\right\| \\
\leq & \mathrm{e}^{\tau|\mathrm{S}|}\left\|\left(\prod_{\varepsilon, \sigma}\left(\phi_{\sigma}^{\varepsilon}\right)^{n_{\sigma}^{\varepsilon}}\right) F_{\tau \gamma}\right\| .
\end{aligned}
$$

In the case of IDB more manipulations are needed. We write

$$
\begin{aligned}
& \left\|\left(\prod_{\varepsilon, \sigma}\left(\frac{\partial}{\partial \phi_{\sigma}^{\varepsilon}}\right)^{n_{\sigma}^{\varepsilon}}\right) \mathrm{e}^{\tau^{\prime} \Phi^{+} \Phi^{-}} \hat{v}\left(\Phi^{+} \Phi^{-}\right)\right\| \\
= & \left\|\left(\prod_{\varepsilon, \sigma}\left(\frac{\partial}{\partial \phi_{\sigma}^{\varepsilon}}\right)^{n_{\sigma}^{\varepsilon}}\right) \mathrm{e}^{-\left(\tau-\tau^{\prime}\right) \Phi^{+} \Phi^{-}} F_{\tau \gamma}(\Phi)\right\| \\
\leq & \sum_{\underline{\ell}}\left(\frac{n}{\ell}\right)\left\|\partial \frac{\underline{\ell}}{\phi} \mathrm{e}^{-\left(\tau-\tau^{\prime}\right) \Phi^{+} \Phi^{-}}\right\|\left\|\partial_{\phi}^{\frac{n}{\phi}-\underline{\ell}} F_{\tau \gamma}(\Phi)\right\|,
\end{aligned}
$$

where we have abridged the notation in an intuitive way: $\underline{n}$ and $\underline{\ell}$ stand for $\left\{n_{\sigma}^{\varepsilon}\right\}$ and $\left\{\ell_{\sigma}^{\varepsilon}\right\}$ respectively, $\left(\frac{n}{\ell}\right)=\prod_{\varepsilon, \sigma}\left(\begin{array}{c}n_{\sigma}^{\varepsilon} \\ \ell_{\sigma}^{\varepsilon}\end{array}\right), \partial_{\phi}^{\underline{\ell}}=\prod_{\varepsilon, \sigma}\left(\partial / \partial \phi_{\sigma}^{\varepsilon}\right)^{\ell_{\sigma}^{\varepsilon}}$ and $\partial_{\phi}^{\underline{n}-\underline{\ell}}=$ $\prod_{\varepsilon, \sigma}\left(\partial / \partial \phi_{\sigma}^{\varepsilon}\right)^{n_{\sigma}^{\varepsilon}-\ell_{\sigma}^{\varepsilon}}$. The derivative of the exponential can be explicitly computed and by using the properties of the Grassmann norms and the bound $\left[\left(\tau-\tau^{\prime}\right)|\phi|^{2}\right]^{m} \leq$

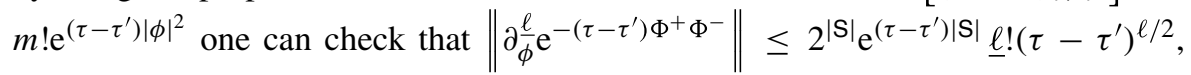
where $\underline{\ell} !=\prod_{\varepsilon, \sigma} \ell_{\sigma}^{\varepsilon}$ ! and $\ell=\sum_{\varepsilon, \sigma} \ell_{\sigma}^{\varepsilon}$. If $F_{\tau \gamma}$ satisfies IDB, then integration of (A.7) in $\mathrm{d} \phi$ proves that for any $\tau^{\prime} \in[0, \tau] F_{\tau^{\prime} \gamma}$ indeed satisfies IDB for some $K, M$ and $p$ depending on $\tau$.

Acknowledgements I express my sincere gratitude to Marcello Porta for inspiring discussions and valuable guidance. This work has been supported by the Swiss National Science Foundation via the grant "Mathematical Aspects of Many-Body Quantum Systems" and by the European Research Council (ERC) under the European Union's Horizon 2020 research and innovation programme, ERC Starting Grant MaMBoQ, Grant Agreement No. 802901. I thank the anonymous referee for the numerous useful comments on a previous version of the paper.

Funding Open Access funding provided by Universität Zürich

Open Access This article is licensed under a Creative Commons Attribution 4.0 International License, which permits use, sharing, adaptation, distribution and reproduction in any medium or format, as long as you give appropriate credit to the original author(s) and the source, provide a link to the Creative Commons 
licence, and indicate if changes were made. The images or other third party material in this article are included in the article's Creative Commons licence, unless indicated otherwise in a credit line to the material. If material is not included in the article's Creative Commons licence and your intended use is not permitted by statutory regulation or exceeds the permitted use, you will need to obtain permission directly from the copyright holder. To view a copy of this licence, visit http://creativecommonshorg/licenses/by/4. $0 \%$

\section{References}

1. Abdesselam, A., Rivasseau, V.: Trees, forests and jungles: A botanical garden for cluster expansions. In: Constructive Physics Results in Field Theory, Statistical Mechanics and Condensed Matter Physics. Springer (1995)

2. Aizenman, M.: Localization at weak disorder: some elementary bounds. Rev. Math. Phys. 6, 1163 (1994)

3. Aizenman, M., Molchanov, S.: Localization at large disorder and at extreme energies: An elementary derivation. Commun. Math. Phys. 157, 245-278 (1993)

4. Aizenman, M., Schenker, J.H., Friedrich, R.M., Hundertmark, D.: Finite-volume fractional-moment criteria for anderson localization. Commun. Math Phys. 224, 219-253 (2001)

5. Aizenman, M., Warzel, S.: Random Operators. Disorder Effects on Quantum Spectra and Dynamics. Graduate Studies in Mathemathics, vol. 168. American Mathematical Society, Providence, RI (2015)

6. Antinucci, G., Fresta, L., Porta, M.: A Supersymmetric hierarchical model for weakly disordered $3 d$ semimetals. Ann Henri Poincaré 21, 3499-3574 (2020)

7. Battle, G., Federbush, P.: A phase cell cluster expansion for Euclidean field theories. Ann. Phys. 142, 95-139 (1982)

8. Bauerschmidt, R., Brydges, D.C., Slade, G.: Introduction to a renormalisation group method

9. Berezin, F.A.: The Methods of Second Quantization. Academic Press, New York (1966)

10. Berezin, F.A.: Introduction to Superanalysis. Springer, Berlin (1987)

11. Berezin, F.A., Marinov, M.S.: Particle spin dynamics as the Grassmann variant of classical mechanics. Ann. Phys. 104, 336-362 (1977)

12. Berline, N., Getzler, E., Vergne, M.: Heat Kernels and Dirac Operators. Springer, Berlin (1992)

13. Blau, M., Thompson, G.: Localization and diagonalization: A review of functional integral techniques for low-dimensional gauge theories and topological field theories. J. Math. Phys. 36, 2192-2236 (1995)

14. Bovier, A.: The density of states in the Anderson model at weak disorder: A renormalization group analysis of the hierarchical model. J Stat Phys. 59, 745-779 (1990)

15. Bovier, A., Campanino, M., Klein, A., Perez, J.F.: Smoothness of the density of states in the anderson model at high disorder. Commun. Math Phys. 114, 439-461 (1988)

16. Brydges, D.C.: A short course on cluster expansions. In: Les Houches Summer School in Theoretical Physics, Session XLIII: Critical Phenomena, Random Systems, Gauge Theories (1984)

17. Brydges, D., Kennedy, T.: Mayer expansions and the Hamilton-Jacobi Equation. J. Stat. Phys. 48, 19 (1987)

18. Campanino, M., Klein, A.: A supersymmetric transfer matrix and differentiability of the density of states in the one-dimensional Anderson model. Comm. Math. Phys. 104, 227-241 (1986)

19. Constantinescu, F., Felder, G., Gawedzki, K., Kupiainen, A.: Analyticity of density of states in a gauge-invariant model for disordered electronic systems. J. Stat Phys. 48, 365-391 (1987)

20. Constantinescu, F., Fröhlich, J., Spencer, T.: Analyticity of the density of states and replica method for random Schrödinger operators on a lattice. J. Stat. Phys. 34 (1984)

21. Disertori, M., Lager, M.: Density of states for random band matrices in two dimensions. Ann. Henri Poincaré 18, 2367-2413 (2017)

22. Disertori, M., Lager, M.: Supersymmetric polar coordinates with applications to the Lloyd Model. Math. Phys. Anal Geom. 23, 2 (2020)

23. Disertori, M., Pinson, H., Spencer, T.: Density of states for random band matrices. Comm. Math Phys. 232, 83-124 (2002) 
24. Disertori, M., Spencer, T.: Anderson localization for a supersymmetric sigma model. Comm. Math Phys. 300, 659-671 (2010)

25. Disertori, M., Spencer, T., Zirnbauer, M.: Quasi-diffusion in a 3D supersymmetric hyperbolic sigma model. Comm. Math Phys. 300, 435-486 (2010)

26. Edwards, S., Thouless, D.: Regularity of the density of states in Anderson's localized electron model. J. Phys. C 4, 453-457 (1971)

27. Efetov, K.B.: Supersymmetry method in localization theory. Soy. Phys. JETP 55, 514-521 (1982)

28. Efetov, K.B.: Anderson localization and supersymmetry. In: 50 Years of Anderson Localization. World Scientific (2010)

29. Elgart, A.: Lifshitz tails and localization in the three-dimensional Anderson model. Duke Math. J. 146, 331-360 (2009)

30. Feldman, J., Knörrer, H., Trubowitz, E.: Fermionic Functional Integrals and the Renormalization Group. CRM Monograph Series 16. American Mathematical Society, Providence, RI (2002)

31. Feldman, J., Knörrer, H., Trubowitz, E.: Convergence of perturbation expansions in Fermionic models. Part 1 Nonperturbative Bounds. Commun. Math. Phys. 247, 195-242 (2004)

32. Fröhlich, J., Spencer, T.: Absence of diffusion in the Anderson tight binding model for large disorder or low energy. Commun. Math Phys. 88, 151-184 (1983)

33. Gawędzki, K., Kupiainen, A.: Gross-Neveu model through convergent perturbation expansion. Comm. Math. Phys. 102 (1985)

34. Gentile, G., Mastropietro, V.: Renormalization group for one-dimensional fermions. A review on mathematical results. Phys. Rep. 352, 273-437 (2001)

35. Giuliani, A., Mastropietro, V.: The two-dimensional Hubbard model on the honeycomb lattice. Commun. Math. Phys 293, 301 (2010)

36. Hörmander, L.: The Analysis of Linear Partial Differential Operators I. Distribution Theory and Fourier Analysis. Springer, Berlin (1990)

37. Johnson, S.G.: Saddle-point integration of $C^{\infty}$ "bump" functions. arXiv: 1508.04376

38. Klein, A., Martinelli, F., Perez, J.F.: A rigorous replica trick approach to Anderson localization in one dimension. Comm. Math. Phys. 106 (1986)

39. Klein, A., Perez, J.F.: On the density of states for random potentials in the presence of a uniform magnetic field. Nucl. Phys. B 251, 199-211 (1985)

40. Klopp, F.: Weak disorder localization and Lifshitz tails. Commun. Math. Phys. 232, 125-155 (2002)

41. Klopp, F., Wolff, T.: Lifshitz tails for 2-dimensional random Schrödinger operators. J. Anal. Math. 88, 63 (2002)

42. Lesniewski, A.: Effective action for the Yukawa 2 quantum field theory. Comm. Math. Phys. 108, 437-467 (1987)

43. Mastropietro, V.: Non-perturbative Renormalization. World Scientific, Singapore (2008)

44. Parisi, G., Sourlas, N.: Random Magnetic Fields, Supersymmetry, and Negative Dimensions. Phys. Rev. Lett. 43, 744 (1979)

45. Parisi, G., Sourlas, N.: Supersymmetric field theories and stochastic differential equations. Nucl. Phys. B 206, 321-332 (1982)

46. Rivasseau, V., Wang, Z.: How to resum Feynman Graphs. Ann. Henri Poincaré, 15, 2069-2083 (2014)

47. Salmhofer, M.: Renormalization: An Introduction. Springer, Berlin (1999)

48. Samuel, S.: The use of anticommuting variable integrals in statistical mechanics. I. The computation of partition functions. J. Math. Phys. 21, 2806 (1980)

49. Samuel, S.: The use of anticommuting variable integrals in statistical mechanics. I. The computation of correlation functions. J. Math. Phys. 21, 2815 (1980)

50. Schäfer, L., Wegner, F.: Disordered system with $\mathrm{n}$ orbitals per site: Lagrange formulation, hyperbolic symmetry, and Goldstone modes. Z. Phys. B 38, 113-126 (1980)

51. Schwarz, A., Zaboronsky, O.: Supersymmetry and localization. Comm. Math. Phys. 183, 463-476 (1997)

52. Shamis, M.: Density of states for Gaussian unitary ensemble, Gaussian orthogonal ensemble, and interpolating ensembles through supersymmetric approach. J. Math. Phys. 54 (2013)

53. Shcherbina, M., Shcherbina, T.: Transfer operator approach to $1 \mathrm{~d}$ random band matrices. arXiv: 1905.08252

54. Shcherbina, M., Shcherbina, T.: Universality for $1 \mathrm{~d}$ random band matrices. arXiv:1910.02999

55. Shcherbina, T.: Universality of the local regime for the block band matrices with a finite number of blocks. J. Stat. Phys. 155, 466-499 (2014) 
56. Simon, B.: Kotani theory for one dimensional Stochastic Jacobi Matrices. Commun. Math. Phys. 89, 227-234 (1983)

57. Sjöstrand, J., Wang, W.-M.: Supersymmetric measures and maximum principles in the complex domain. Exponential decay of Green's functions. Annales scientifiques de l'É,.N.S. $4^{e}$ série 32, 347-414 (1999)

58. Sjöstrand, J., Wang, W.-M.: Exponential decay of averaged Green functions for random Schrödinger operators. A direct approach. Annales scientifiques de l'É,.N.S. $4^{e}$ série 32, 415-431 (1999)

59. Spencer, T.: Lifshitz tails and localization. Preprint (1993)

60. Spencer, T.: SUSY statistical mechanics and random band matrices. In: Giuliani, A., Mastropietro, V., Yngvason, J. (eds.) Quantum Theory from Small to Large Scales: Lecture Notes of the Les Houches Summer School: Volume 95 (2010), and In: Quantum Many Body Systems Cetraro, Italy 2010 (2012)

61. Wang, W.-M.: Localization and universality of Poisson statistics for the multidimensional Anderson model at weak disorder. Invent. Math. 146, 365-398 (2001)

62. Wegner, F.: The mobility edge problem: continuous symmetry and a conjecture. Z. Phys. B 35, 207$210(1979)$

63. Wegner, F.: Bounds on the density of states of disordered systems. Z. Physik B 44 (1981)

64. Wegner, F.: Supermathematics and Its Applications to Statistical Physics. Lecture Notes in Physics. pp. 920 (2016)

Publisher's Note Springer Nature remains neutral with regard to jurisdictional claims in published maps and institutional affiliations. 